\title{
Total Synthesis of (+)-Raputindole A: An Iridium-catalyzed Cyclization Approach
}

\author{
Juliana L. L. F. Regueira ${ }^{,}{ }^{\dagger}$, Luiz F. Silva Jr ${ }^{\psi, \#}$, Ronaldo A. Pilli*, ${ }^{*}$ \\ ${ }^{\psi}$ Institute of Chemistry, University of São Paulo (USP), 05508-000 São Paulo, Brazil. ' Institute of Chemistry, \\ University of Campinas, 13083-970 Campinas, São Paulo, Brazil.
}

\section{Supporting Information}

Table of Contents

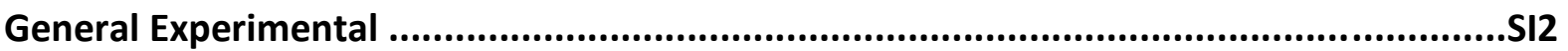

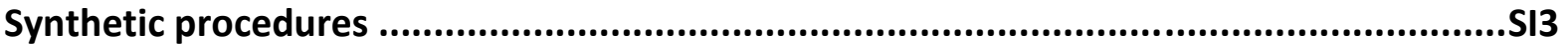

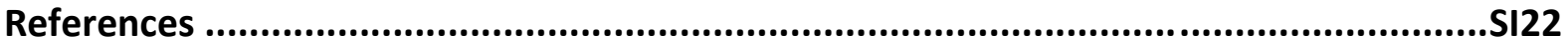

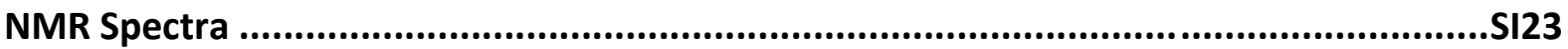




\section{General Experimental}

All the solvents were kept under a nitrogen atmosphere. Dimethylformamide (DMF) and TMEDA were distilled after 12 hours under $4 \AA$ molecular sieves. Tetrahydrofuran (THF) was distilled under sodium-benzophenone immediately prior to use. ${ }^{1}$ Dichloroethane (DCE), dichloromethane (DCM) and trimethylamine $\left(\mathrm{Et}_{3} \mathrm{~N}\right)$ were distilled from calcium hydride under a nitrogen atmosphere prior to use. The other anhydrous solvents were used without further purification, unless otherwise specified. The commercial reagents were used without further purification, unless otherwise stated. Oven-dried syringes were used to transfer the anhydrous solvents. The progress of the reactions was monitored by thin chromatography (TLC) using $0.25 \mathrm{~mm}$ E. Merck silica plates $60-\mathrm{F} 254$ or neutral alumina. The visualizing agents were UV light (254 nm), p-anisaldehyde, curcumin and/or phosphomolybdic acid stain solutions and heat as developing agents. The purifications of the crude reaction mixtures were carried out using flash silica gel chromatography (Merck, 40-63 $\mu \mathrm{m}$ ) under positive pressure. NMR spectra were recorded on Bruker Alll 300 or $500 \mathrm{MHz}$, Varian Gemini $400 \mathrm{MHz}$ and Bruker Alll $800 \mathrm{MHz}$ equipped with with cryoprobe. Chemical shifts $(\delta)$ are in parts per million (ppm) and the calibration was performed with reference to residual non-deuterated solvent $\left(\mathrm{CHCl}_{3}{ }^{1} \mathrm{H}\right.$ NMR $\delta 7.26 \mathrm{ppm},{ }^{13} \mathrm{C}$ NMR $\left.77.0 \mathrm{ppm}\right)$. The following descriptors were used to describe the NMR data: chemical shift (ppm), multiplicity ( $b r=$ broad, $s=$ singlet, $d=$ doublet, $\mathrm{t}=$ triplet, $\mathrm{q}=$ quartet, $\mathrm{m}=$ multiplet), coupling constant (Hertz) and integration. Infrared spectra were recorded on a Thermo Scientific iD3 spectrometer. High resolution mass spectra (HRMS) were recorded in a MicroToF Bruker Daltonics and in an Agilent

Technologies 6530 Accurate-Mass Q-TOF LC-MS. The specific optical rotations were measured on a Perkin Elmer 341 polarimeter (sodium line $=589.3 \mathrm{~nm}$ ). 


\section{Synthetic procedures}

\section{Methyl 5-bromo-1-tosyl-1H-indole-6-carboxylate (8b)}

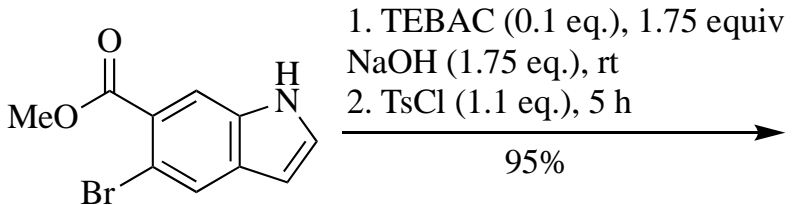

8<smiles>COC(=O)c1cc2c(ccn2-c2ccccc2)[nH]1</smiles>

$\mathbf{8 b}$

To a soln. of indole 8 (1.00 g, $3.94 \mathrm{mmol}, 1.0$ eq.) in DCM (150 mL) was added TEBAC (0.092 g, $0.39 \mathrm{mmol}, 0.1$ eq.). Then $\mathrm{NaOH}(0.276 \mathrm{~g}, 6.89 \mathrm{mmol}, 1.75$ eq.) and $\mathrm{TsCl}(0.826 \mathrm{~g}, 4.33$ mmol, 1.1 eq.) were successively added. The reaction flask was stirred at room temperature for 5 hours. The reaction mixture was quenched with $\mathrm{H}_{2} \mathrm{O}(50 \mathrm{~mL})$ and $1 \mathrm{M}$ hydrochloric acid $(30 \mathrm{~mL})$ and the resulting mixture was stirred for $10 \mathrm{~min}$. The aqueous layer was separated and extracted with 3\% methanol/chloroform soln. $(3 \times 100 \mathrm{~mL})$. The combined organic layer was washed with brine, dried over $\mathrm{MgSO}_{4}$ and evaporated under reduced pressure. The resulting residue was purified by flash column chromatography ( $\mathrm{SiO}_{2}, 10-20 \% \mathrm{AcOEt} /$ hexanes) to provide $8 \mathrm{~b}$ in $95 \%$ yield $(1.53 \mathrm{~g}, 3.75 \mathrm{mmol})$ as a white solid.

${ }^{1} \mathrm{H}$ NMR (800 MHz, $\left.\mathrm{CDCl}_{3}\right): \delta 8.46(\mathrm{~s}, 1 \mathrm{H}) ; 7.81(\mathrm{~s}, 1 \mathrm{H}) ; 7.76(\mathrm{~d}, J=8.0 \mathrm{~Hz} ; 2 \mathrm{H}) ; 7.67$ (d, J = 4.0 $\mathrm{Hz} ; 1 \mathrm{H}) ; 7.25$ (d, J = $8.0 \mathrm{~Hz} ; 2 \mathrm{H}) ; 6.61(\mathrm{dd}, \mathrm{J}=0.8$ and $3.2 \mathrm{~Hz} ; 1 \mathrm{H}) ; 3.98(\mathrm{~s}, 3 \mathrm{H}) ; 2.36(\mathrm{~s}, 3 \mathrm{H})$. ${ }^{13} \mathrm{C}$ NMR (200 MHz, $\left.\mathrm{CDCl}_{3}\right): \delta 166.7 ; 145.6 ; 134.8 ; 134.3 ; 133.1 ; 130.2 ; 130.0 ; 127.6 ; 126.8$; $126.7 ; 116.6 ; 115.6 ; 108.0 ; 52.7 ; 21.6$.

IR (cm $\left.{ }^{-1}\right) 1727 ; 1593 ; 1452 ; 1425 ; 1403 ; 1374 ; 1279 ; 1222 ; 1169 ; 1101 ; 1010 ; 982 ; 930 ; 889$; $864 ; 815 ; 768 ; 727 ; 708$.

HRMS (ESI) m/z [M+H] $]^{+}$Calcd for $\mathrm{C}_{17} \mathrm{H}_{15} \mathrm{NO}_{4} \mathrm{SBr}$ 407.9905; Found 407.9887.

Mp: $155.0-155.8^{\circ} \mathrm{C}$. 
<smiles>COC(=O)c1cc2c(ccn2-c2ncccc2Br)cc1Br</smiles>

8b

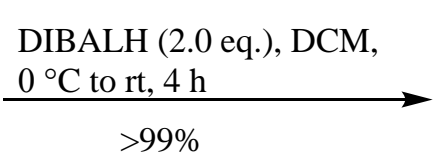

$>99 \%$<smiles></smiles>

10

To a flame dried Schlenk flask was added $8 \mathbf{b}(0.520 \mathrm{~g}, 1.28 \mathrm{mmol}, 1.0$ eq.) and DCM (10 mL). The soln. was cooled to $0{ }^{\circ} \mathrm{C}$ and then DIBALH (1M in toluene, $2.54 \mathrm{~mL}, 2.54 \mathrm{mmol}, 2.0$ eq.) was slowly added. The reaction mixture was stirred for 1 hour and the temperature was let to reach room temperature. After 3 hours, the reaction was completed by TLC and the reaction temperature was decreased to $0{ }^{\circ} \mathrm{C}$. AcOEt $(8 \mathrm{~mL})$ was slowly added, followed by the addition of Rochelle's salt soln. (1M soln., $8 \mathrm{~mL}, 8 \mathrm{mmol})$ and $\mathrm{Et}_{2} \mathrm{O}(40 \mathrm{~mL})$. The reaction mixture was stirred overnight and then the aqueous layer was separated and extracted with $3 \% \mathrm{MeOH}$ in chloroform soln. $(3 \times 15 \mathrm{~mL})$. The combined organic layer was washed with brine, dried $\left(\mathrm{MgSO}_{4}\right)$ and the solvent was removed under reduced pressure. The product 10 was obtained in $>99 \%$ yield $(0.484 \mathrm{~g}, 1.27 \mathrm{mmol})$ as a white solid.

${ }^{1} \mathrm{H}$ NMR (300MHz, $\left.\mathrm{CDCl}_{3}\right) \delta 8.10(\mathrm{~s}, 1 \mathrm{H}) ; 7.77(\mathrm{~s}, 1 \mathrm{H}) ; 7.73(\mathrm{~d}, \mathrm{~J}=9.0 \mathrm{~Hz}, 2 \mathrm{H}), 7.57(\mathrm{~d}, \mathrm{~J}=6.0$ $\mathrm{Hz}, 1 \mathrm{H}) ; 7.24(\mathrm{~d}, \mathrm{~J}=9.0 \mathrm{~Hz}, 2 \mathrm{H}) ; 6.59(\mathrm{dd}, \mathrm{J}=0.3$ and $3.6 \mathrm{~Hz}, 1 \mathrm{H}) ; 4.83(\mathrm{~s}, 2 \mathrm{H}) ; 2.35(\mathrm{~s}, 3 \mathrm{H})$.

${ }^{13} \mathrm{C}$ NMR (126 MHz, d6-(CD $\left.)_{2} \mathrm{CO}\right): \delta 146.3,138.0,135.7,135.0,131.8,130.7,128.4,127.5$, $125.2,116.7,113.4,108.9,64.3,21.2$.

HRMS (ESI) $\mathbf{m} / \mathbf{z}$ [M- $\left.\mathrm{H}_{2} \mathrm{O}+\mathrm{H}\right]^{+}$Calcd for $\mathrm{C}_{16} \mathrm{H}_{12} \mathrm{NO}_{2} \mathrm{SBr} 361.9850$; Found= 361.9863 .

Mp: $136,7-139,2^{\circ} \mathrm{C}$.

IR (cm-1): 1411; 1367; 1164; 1123; 1057; 812; 688.

\section{5-Bromo-1-tosyl-1H-indole-6-carbaldehyde (11)}

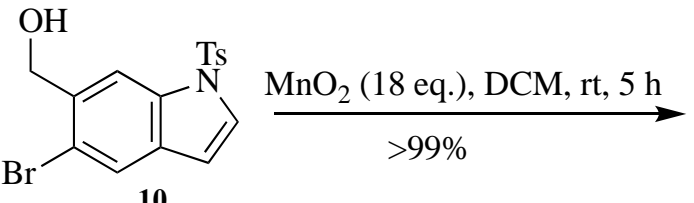

10<smiles>O=Cc1cc2[n-]ccc2cc1Br</smiles>

11

In a round bottom flask was added a solution of 10 (1.164 g, $3.063 \mathrm{mmol}, 1.0$ eq.) in DCM (100 $\mathrm{mL}$ ). $\mathrm{MnO}_{2}$ (5.205 g, $55.10 \mathrm{mmol}, 18$ eq.) was then added and the mixture was stirred for at rt $5 \mathrm{~h}$. The suspension was filtered in a Celite pad and washed with AcOEt (400 mL) After 
evaporation under reduced pressure, aldehyde 11 was obtained in $>99 \%$ yield $(1.15 \mathrm{~g}, 3.06$ mmol) as an off-white solid.

${ }^{1} \mathrm{H}$ NMR (300MHz, $\left.\mathrm{CDCl}_{3}\right): \delta 10.42(\mathrm{~s}, 1 \mathrm{H}) ; 8.53(\mathrm{~s}, 1 \mathrm{H}) ; 7.82-7.76(\mathrm{~m}, 4 \mathrm{H}) ; 7.27(\mathrm{~m}, 1 \mathrm{H}) ; 7.25$ (m, $1 \mathrm{H}) ; 6.64-6.63$ (dd, J=0.87 and 3.7 Hz; $1 \mathrm{H}) ; 2.35$ (s, $3 \mathrm{H})$.

${ }^{13} \mathrm{C}$ NMR (75 MHz, $\left.\mathrm{CDCl}_{3}\right): \delta$ 191.7, 145.7, 136.5, 134.6, 133.6, 131.3, 130.2, 129.2, 127.0, $125.9,120.5,115.1,107.7,21.6$.

IR (cm $\left.{ }^{-1}\right):$ 1686, 1598, 1417, 1276, 1167, 1090, 815, 765, 664.

$\mathrm{Mp}=191.0-191.3^{\circ} \mathrm{C}$

HRMS (ESI-TOF) $\boldsymbol{m} / \mathbf{z}[\mathrm{M}+\mathrm{H}]^{+}$Calcd for $\mathrm{C}_{16} \mathrm{H}_{13} \mathrm{NO}_{3} \mathrm{SBr} 377.9799$; Found 377.9779.

5-(4,4,5,5-Tetramethyl-1,3,2-dioxaborolan-2-yl)-1-tosyl-1H-indole-6-carbaldehyde (12)<smiles>O=Cc1cc2[nH]ccc2cc1Br</smiles>

11

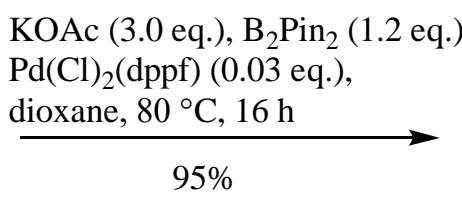

$95 \%$<smiles>CC1(C)OB(c2cc3cc[n-]c3cc2C=O)OC1(C)C</smiles>

In a Schlenk flask charged with aldehyde $11(1.014 \mathrm{~g}, 2.69 \mathrm{mmol}, 1.0$ eq.), bis(pinacolato)diboron (0.842 g, $3.22 \mathrm{mmol}, 1.2 \mathrm{eq}$.), KOAc (0.790 g, $8.04 \mathrm{mmol}, 3.0$ eq.) and $\mathrm{Pd}(\mathrm{Cl})_{2}(\mathrm{dppf})(0.059 \mathrm{~g}, 0.080 \mathrm{mmol}, 0.03 \mathrm{eq}$.) under nitrogen atmosphere was added degassed dioxane $(36 \mathrm{~mL})$. The reaction mixture was stirred at $80^{\circ} \mathrm{C}$ using an oil bath for 16 hours. After this time, the reaction mixture was cooled to $\mathrm{rt}$ and the crude mixture was filtered in a Celite $/ \mathrm{SiO}_{2}$ pad and washed with AcOEt $(300 \mathrm{~mL})$. The solvent was removed under reduced pressure and the crude product was purified by flash column chromatography $\left(\mathrm{SiO}_{2}, 10-20 \%\right.$ AcOEt/hexanes) to provide 12 in $95 \%$ yield $(1.05 \mathrm{~g}, 2.55 \mathrm{mmol})$ as an off-white thick oil.

${ }^{1} \mathrm{H}$ NMR (400MHz, $\left.\mathrm{CDCl}_{3}\right): \delta 10.57(\mathrm{~s}, 1 \mathrm{H}) ; 8.56(\mathrm{t}, \mathrm{J}=0.8$ and $1.2 \mathrm{~Hz}, 1 \mathrm{H}) ; 8.02(\mathrm{~s}, 1 \mathrm{H}) ; 7.78$ (d, J = 8.4 Hz; $2 \mathrm{H}) ; 7.74$ (d, J = 3.6 Hz; $1 \mathrm{H}) ; 7.22$ (d, J = 8.4 Hz, $2 \mathrm{H}) ; 6.71$ (dd, J = 1.2 and 3.6 $\mathrm{Hz} ; 1 \mathrm{H}) ; 2.32$ (s, $3 \mathrm{H}) ; 1.39$ (s, $12 \mathrm{H})$.

${ }^{13} \mathrm{C}$ NMR (75 MHz, $\left.\mathrm{CDCl}_{3}\right): \delta$ 194.0, 145.4, 137.8, 135.7, 134.8, 134.4, 130.0, 130.0, 129.0, 126.8, 114.0, 108.9, 84.3, 25.0, 24.8, 21.5. 
HRMS (ESI) $m / z[M+H]^{+}$Calcd for $\mathrm{C}_{22} \mathrm{H}_{25} \mathrm{BNO}_{5} \mathrm{~S}^{+}$426.1541; Found 426.1570.

IR (cm $\left.{ }^{-1}\right):$ 2978, 1680, 1477, 1455, 1414, 1373, 1331, 1279, 1169, 1120, 974, 853, 675.

(6-Formyl-1-tosyl-1H-indol-5-yl)boronic acid (7)

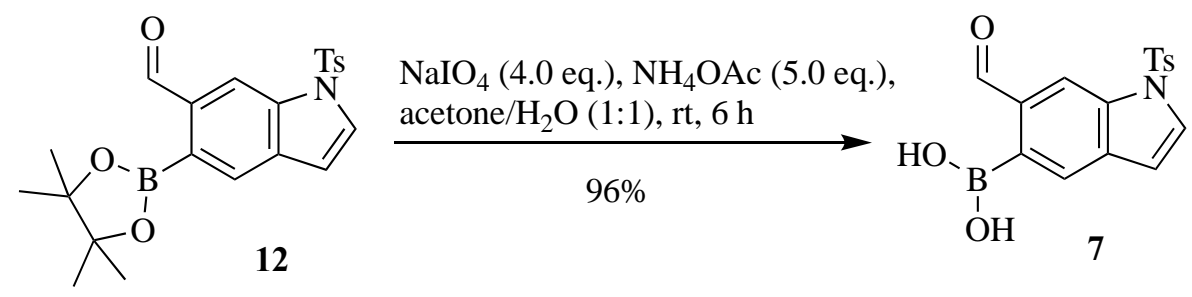

In a round bottom flask was added the aldehyde $12(0.165 \mathrm{~g}, 0.388 \mathrm{mmol}, 1.0 \mathrm{eq}$.) dissolved in acetone $\left./ \mathrm{H}_{2} \mathrm{O}(1: 1, \mathrm{v} / \mathrm{v}) ,8 \mathrm{~mL}\right)$. $\mathrm{NaIO}_{4}\left(0.333 \mathrm{~g}, 1.56 \mathrm{mmol}, 4.0\right.$ eq.) and $\mathrm{NH}_{4} \mathrm{OAc}(0.150 \mathrm{~g}$, $1.94 \mathrm{mmol}, 5.0$ eq.) were added and the reaction mixture was stirred $6 \mathrm{~h}$ at $\mathrm{rt}$. The solvent was removed under reduced pressure and the product was extracted using a mixture of $5 \%$ $\mathrm{MeOH} / \mathrm{DCM}(3 \times 10 \mathrm{~mL})$. The combined organic layer was washed with brine, dried over $\mathrm{Na}_{2} \mathrm{SO}_{4}$ and the solvent was reduced under reduced pressure to provide 7 in $96 \%$ yield as an orange solid $(0.128 \mathrm{~g}, 0.373 \mathrm{mmol})$.

${ }^{1} \mathrm{H}$ NMR (300 MHz, CDCl 3$): \delta 9.97(\mathrm{~s}, 1 \mathrm{H}) ; 8.54(\mathrm{~s}, 1 \mathrm{H}) ; 8.50(\mathrm{~s}, 1 \mathrm{H}) ; 7.82(\mathrm{~m}, 2 \mathrm{H}) ; 7.78(\mathrm{~s}, 1 \mathrm{H})$; $7.28(\mathrm{~m}, 1 \mathrm{H}) ; 7.18(\mathrm{~s}, 1 \mathrm{H}) ; 6.81(\mathrm{~m}, 1 \mathrm{H}) ; 2.37(\mathrm{~s}, 3 \mathrm{H})$.

${ }^{13}$ C NMR (75 MHz, d6-(CD $\left.)_{2} \mathrm{CO}\right): \delta$ 196.9, 147.6, 138.6, 136.4, 136.5, 136.3, 132.2, 131.9, $130.4,128.7,128.6,120.3,111.0,22.2$.

HRMS (ESI) $\mathbf{m} / \mathbf{z}$ [M-H $\mathrm{H}_{2}$ ] $^{-}$Calcd for $\mathrm{C}_{16} \mathrm{H}_{13} \mathrm{BNO}_{5} \mathrm{~S}$ 326.0658; Found 326.0655.

$M p=135-139^{\circ} \mathrm{C}$.

(5R,7R) and (5S,7S)-5-Methyl-1-tosyl-5-vinyl-1,5,6,7-

tetrahydrocyclopenta[f]indol-7-ol ((+/-)-6)

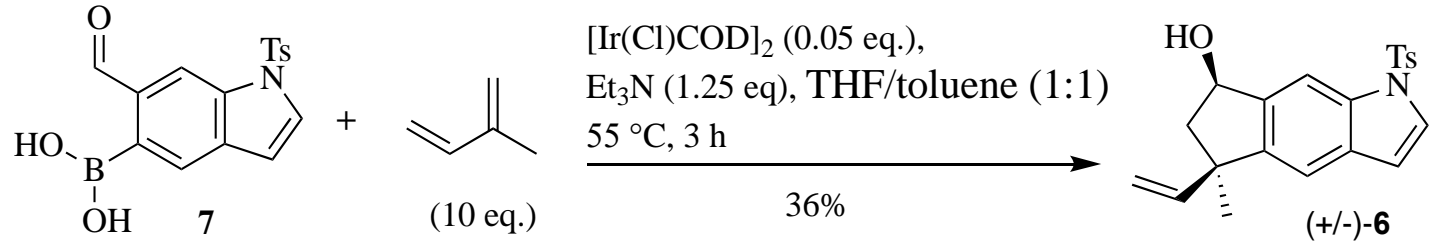


A resealable pressured tube equipped with a magnetic stir bar was charged with $[\operatorname{Ir}(\mathrm{Cl})(\mathrm{COD})]_{2}$ (0.001 g, $0.001 \mathrm{mmol}, 0.05$ eq.) and degassed toluene (0.3 mL). Et $3 \mathrm{~N}(0.0050 \mathrm{~mL}, 0.036 \mathrm{mmol}$, 1.25 eq.) and isoprene ( $0.030 \mathrm{~mL}, 0.29 \mathrm{mmol}, 10$ eq.) were next added by a syringe. In another vessel, a solution of aldehyde 7 ( $0.010 \mathrm{~g}, 0.029 \mathrm{mmol}, 1.0$ eq.) in THF/toluene $(0.7 \mathrm{~mL}, 5: 2 \mathrm{v} / \mathrm{v}$ ) which was cannulated to the resealable tube and the reaction mixture was protect from visible light and the temperature was increased to $55{ }^{\circ} \mathrm{C}$ using a heating block. After $3 \mathrm{~h}$ (monitored by ${ }^{1} \mathrm{H} N M R$ ) the reaction mixture was allowed to cool to $\mathrm{rt}$ and it was concentrated under reduced pressure. The resulting brown mixture was purified by flash column chromatography $\left(\mathrm{SiO}_{2}, 10-20 \%\right.$ EtOAc/hexanes) to provide indole (+/-)-6 in 36\% yield (0.0038 $\mathrm{g}, 0.010 \mathrm{mmol}$ ) as a yellow solid.

${ }^{1} \mathrm{H}$ NMR (300 MHz, CDCl 3$): \delta 8.01(\mathrm{~m}, 1 \mathrm{H}), 7.81(\mathrm{~m}, 2 \mathrm{H}), 7.57(\mathrm{~d}, \mathrm{~J}=6.0 \mathrm{~Hz}, 1 \mathrm{H}), 7.24(\mathrm{~m}, 2 \mathrm{H})$, $7.22(\mathrm{~m}, 1 \mathrm{H}), 6.61(\mathrm{dd}, \mathrm{J}=1.5$ and $6.0 \mathrm{~Hz}, 1 \mathrm{H}), 6.17(\mathrm{~m}, 1 \mathrm{H}), 5.33(\mathrm{~m}, 1 \mathrm{H}), 5.04(\mathrm{~m}, 2 \mathrm{H}), 2.43$ (m, 1H), $2.34(\mathrm{~s}, 3 \mathrm{H}), 2.15(\mathrm{~m}, 1 \mathrm{H}), 1.61(\mathrm{brs}, 1 \mathrm{H}), 1.32(\mathrm{~m}, 3 \mathrm{H})$.

${ }^{13} \mathrm{C}$ NMR (126 MHz, CDCl 3 ): $\delta$ 147.5, 144.9, 144.7, 141.7, 135.4, 134.5, 131. 7, 129.9, 126.9, $126.8,116.1,111.7,109.5,108.8,74.6,51.0,47.9,26.3,21.6$.

HRMS (ESI) $m / z[\mathrm{M}+\mathrm{Na}]^{+}$Calcd for $\mathrm{C}_{21} \mathrm{H}_{21} \mathrm{NO}_{3} \mathrm{SNa} 390.1134$; Found 390.1150 .

$\mathrm{Mp}=84.1-89.9^{\circ} \mathrm{C}$.

IV $\left(\mathrm{cm}^{-1}\right)=2359,2343,1372,1167,1109,703,668$.
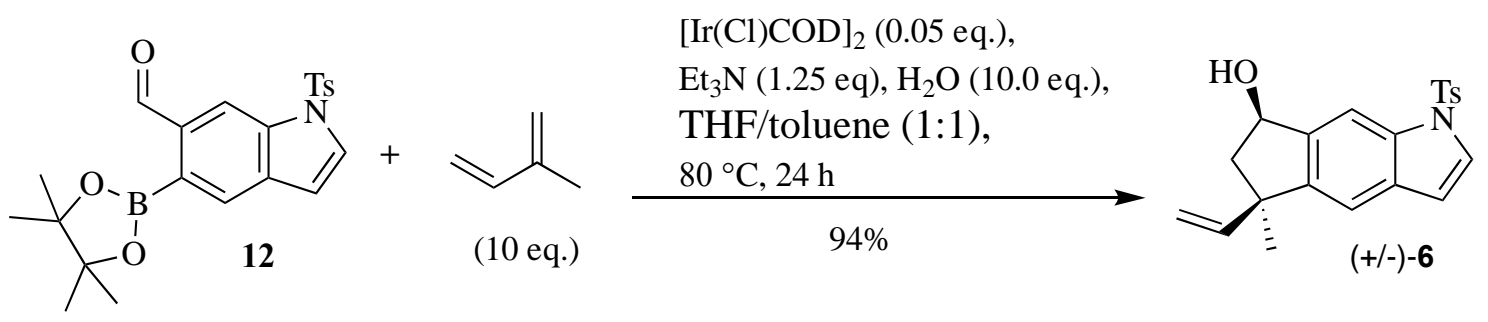

A resealable pressure tube equipped with a magnetic stir bar was charged with $[\operatorname{Ir}(\mathrm{Cl})(\mathrm{COD})]_{2}$ (0.012 g, $0.017 \mathrm{mmol}, 0.05$ eq.) and degassed toluene $(0.7 \mathrm{~mL}) .^{2} \mathrm{Et}_{3} \mathrm{~N}(0.060 \mathrm{~mL}, 0.42 \mathrm{mmol}$, 1.5 eq.) and isoprene $(0.340 \mathrm{~mL}, 3.34 \mathrm{mmol}, 10.0$ eq.) were next added via syringe. In another vessel, a solution of pinacol ester 12 (0.142 g, $0.334 \mathrm{mmol}, 1.0$ eq.) in THF/toluene (3.5 mL, $3: 2 \mathrm{v} / \mathrm{v})$ was prepared and cannulated to the resealable reaction tube. Degassed $\mathrm{H}_{2} \mathrm{O}(0.060$ $\mathrm{mL}, 3.3 \mathrm{mmol}$ ) was added. The reaction flask was protected from visible light and the reaction temperature was increased to $80{ }^{\circ} \mathrm{C}$ using a heating block. After 24 hours (monitored by ${ }^{1} \mathrm{H}$ 
NMR) the reaction mixture was allowed to cool to $\mathrm{rt}$ and concentrated under reduced pressure. The resulting brown mixture was purified by flash column chromatography $\left(\mathrm{SiO}_{2}\right.$, $10-20 \%$ EtOAc/hexanes) to provide indole $(+/-)-6$ in $94 \%$ yield $(0.116 \mathrm{~g}, 0.315 \mathrm{mmol})$ as a yellow solid.

$(5 R, 7 R)$ and $(5 S, 7 S)-5-M e t h y l-1-t o s y l-5-v i n y l-1,5,6,7-t e t r a h y d r o c y c l o p e n t a[f]$ indol-7-yl acetate $((+/-)-13)$<smiles></smiles>

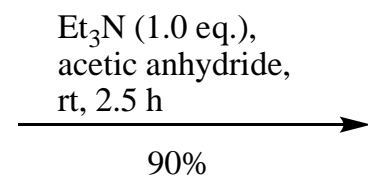

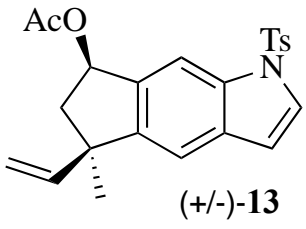

To a solution of indole (+/-)-6 (0.020 g, $0.054 \mathrm{mmol}, 1.0$ eq.) in acetic anhydride (1.0 mL) in a round-bottom flask was added $\mathrm{Et}_{3} \mathrm{~N}(0.0080 \mathrm{~mL}, 0.054 \mathrm{mmol}, 1.0$ eq.). After $2.5 \mathrm{~h}$ of stirring, the mixture was diluted with diethyl ether $(3 \mathrm{~mL})$ and washed with saturated $\mathrm{NaHCO}_{3}(2 \times 3$ $\mathrm{mL}$ ). The organic layer was dried over $\mathrm{MgSO}_{4}$ and the solvent was removed under reduced pressure to provide (+/-)-13 as a yellow oil (0.020 g; $0.049 \mathrm{mmol})$ in $90 \%$ yield. ${ }^{4}$

${ }^{1} \mathrm{H}$ NMR (500 MHz, CDCl 3$): \delta 7.97(\mathrm{~s}, 1 \mathrm{H}), 7.81(\mathrm{~d}, J=8.5 \mathrm{~Hz}, 2 \mathrm{H}), 7.58(\mathrm{~d}, J=3.5 \mathrm{~Hz}, 1 \mathrm{H}), 7.27$ $(\mathrm{m}, 3 \mathrm{H}), 6.62(\mathrm{dd}, J=0.5,3.5 \mathrm{~Hz}, 1 \mathrm{H}), 6.31(\mathrm{~m}, 1 \mathrm{H}), 6.12(\mathrm{~m}, 1 \mathrm{H}), 5.08(\mathrm{~m}, 2 \mathrm{H}), 2.50(\mathrm{~m}, 1 \mathrm{H})$, $2.37(\mathrm{~s}, 3 \mathrm{H}), 2.24(\mathrm{~s}, 1 \mathrm{H}), 2.13(\mathrm{~s}, 3 \mathrm{H}) 1.38(\mathrm{~s}, 3 \mathrm{H})$.

${ }^{13} \mathrm{C}$ NMR (126 MHz, CDCl 3 ) $\delta$ (ppm): 166.38, 146.2, 145.8, 144.9, 137.3, 135.3, 134.3, 132.2, $129.9,127.1,127.0,115.9,111.2,110.4,108.7,76.4,48.1,47.5,26.3,22.2,21.4$.

\section{(5R,7R)-5-Methyl-1-tosyl-5-vinyl-1,5,6,7-tetrahydrocyclopenta[f]indol-7-ol $((R, R)-6)$} and

$(5 S, 7 S)$-5-methyl-1-tosyl-5-vinyl-1,5,6,7-tetrahydrocyclopenta[f]indol-7-yl acetate ((S,S)-

13)<smiles>C=C[C@]1(C)CC(O)c2cc3[nH]ccc3cc21</smiles>

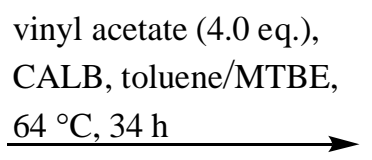

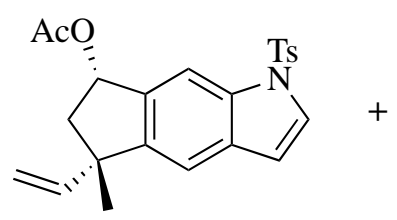

$(S, S)-\mathbf{1 3}(\mathbf{3 0 \%})$<smiles></smiles>

$(R, R)-6(36 \%)$

Table 1. Optimization of enzymatic resolution.

\begin{tabular}{|c|l|l|l|l|l|}
\hline Entry & Solvent & CALB (eq.) & Time $(\mathrm{h})$ & Conversion & e.e. \\
\hline
\end{tabular}




\begin{tabular}{|l|l|l|l|l|l|}
\hline & & & & & \\
\hline 1 & Toluene & 1.2 & 17 & 25 & $>99 \%$ \\
\hline 2 & Toluene:MTBE (4:1) & 1.2 & 17 & 37.5 & $>99 \%$ \\
\hline 3 & Toluene:MTBE (4:1) & 1.2 & 34 & 47.5 & $>99 \%$ \\
\hline 4 & Toluene:MTBE (4:1) & 2.0 & 17 & 40 & $>99 \%$ \\
\hline 5 & Toluene:MTBE (4:1) & 2.0 & 34 & 50 & $>99 \%$ \\
\hline 6 & Toluene:MTBE (4:1) & 2.0 & 34 & $50(30 / 36)^{\text {a }}$ & $>99 \%$ \\
\hline
\end{tabular}

${ }^{a}$ yields of $(S, S)-13 /(R, R)-6$.

To a magnetically stirred solution of alcohol (+/-)-6 $(0.080 \mathrm{~g}, 0.22 \mathrm{mmol}, 1.0 \mathrm{eq}$.$) in a$ toluene/MTBE mixture $(6.40 \mathrm{~mL}, 8: 2 \mathrm{v} / \mathrm{v})$ was added CALB $(0.160 \mathrm{~g}, 2.0 \mathrm{~g} / \mathrm{g}$ alcohol). The reaction mixture was warmed to $64{ }^{\circ} \mathrm{C}$ using a heating block and after 15 minutes, vinyl acetate $(0.080 \mathrm{~mL}, 0.87 \mathrm{mmol}, 4.0$ eq.) was added. After $34 \mathrm{~h}$, the reaction mixture was allowed to cool to rt. The solids were filtered off and washed with EtOAc (40 mL) and DCM $(40 \mathrm{~mL})$. The solvent was removed under reduced pressure and the crude product was purified by flash chromatography $\left(\mathrm{SiO}_{2}, 5-30 \% \mathrm{EtOAc} /\right.$ hexanes). The enantiomerically pure acetylated product $(S, S)-\mathbf{1 3}$ was isolated in $30 \%$ yield $(0.0264 \mathrm{~g}, 0.064 \mathrm{mmol})$ as a colorless thick oil and the enantiomerically pure alcohol $(R, R)-6$, in $36 \%$ yield $(0.029 \mathrm{~g}, 0.079 \mathrm{mmol})$ as a white viscous oil. The enantiomeric excess (ee) of $99 \%$ was determined by HPLC using an analytical column Chiralpak IA.

(5R,7S)-7-allyl-5-methyl-1-tosyl-5-vinyl-1,5,6,7-tetrahydrocyclopenta[f]indole (la) and (5R,7R)-7-allyl-5-methyl-1-tosyl-5-vinyl-1,5,6,7-tetrahydrocyclopenta[f]indole (Ib)
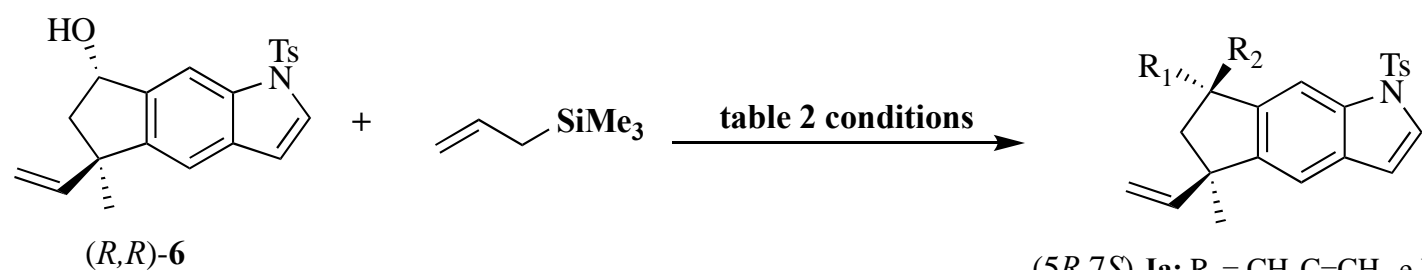

(5R,7S)-Ia: $\mathrm{R}_{1}=\mathrm{CH}_{2} \mathrm{C}=\mathrm{CH}_{2}$ e $\mathrm{R}_{2}=\mathrm{H}$ $(5 R, 7 R)-\mathbf{I b}: \mathrm{R}_{1}=\mathrm{H}, \mathrm{R}_{2}=\mathrm{CH}_{2} \mathrm{C}=\mathrm{CH}_{2}$

Table 2. Screening of Lewis acids in the allylation reaction. 


\begin{tabular}{|l|l|l|l|l|l|}
\hline Entry & \multicolumn{1}{|c|}{ Lewis Acid } & $\begin{array}{c}\text { Loading } \\
\text { catalyst } \\
(\mathrm{mmol})\end{array}$ & \multicolumn{1}{|c|}{ Time (h) } & Ratio la:Ib & Yield (\%) \\
\hline 1 & $\mathrm{InCl}_{3}$ & 20 & 27 & $1: 4$ & 52 \\
\hline 2 & $\mathrm{BiBr}_{3}$ & 10 & 1.5 & $1: 3$ & 66 \\
\hline 3 & $\mathrm{FeCl}_{3}$ & 10 & 1.5 & - & $\begin{array}{l}\text { complex } \\
\text { mixture }\end{array}$ \\
\hline
\end{tabular}

A All the reactions were carried out at $r t$ and using $D C E^{b}$ as the solvent.

To a resealable pressure tube charged with Lewis Acid under nitrogen atmosphere was added a solution of indole (5R,7R)-6 and allyltrimethylsilane in DCE. After 1 hour under stirring at room temperature, the reaction mixture was concentrated under reduced pressure and the crude product was purified by flash chromatography $\left(\mathrm{SiO}_{2}, 5 \% \mathrm{EtOAc} /\right.$ hexanes) to provide an unseparable 1:2 mixture of $(5 R, 7 S)$-la and $(5 R, 7 R)$-lb in yields as indicated in table 2.

${ }^{1} \mathrm{H}$ NMR (CDCl3, solvent) for (5R,7S)-la and (5R,7R)-lb): $\delta 7.81(\mathrm{~s}, 1 \mathrm{H}), 7.77(\mathrm{~d}, J=8.0 \mathrm{~Hz}, 2 \mathrm{H})$, 7.49 (d, $J=3.5 \mathrm{~Hz}, 1 \mathrm{H}), 7.22(\mathrm{~d}, J=8.0 \mathrm{~Hz}, 2 \mathrm{H}), 7.15(\mathrm{~s}, 1 \mathrm{H}),[6.56, \mathrm{~d}, J=4.0 \mathrm{~Hz}$ (major) and 6.59, $d, J=4.0 \mathrm{~Hz}$ (minor), $1 \mathrm{H}], 6.02(\mathrm{~m}, 1 \mathrm{H}), 5.90(\mathrm{~m}, 1 \mathrm{H}), 5.11(\mathrm{~m}, 4 \mathrm{H}),[3.40, \mathrm{~m}$ (major) and 3.29, $m$ (minor), $1 \mathrm{H}], 2.74(\mathrm{~m}, 1 \mathrm{H}), 2.35(\mathrm{~s}, 3 \mathrm{H}), 2.32(\mathrm{~m}, 1 \mathrm{H}), 2.14(\mathrm{~m}, 1 \mathrm{H}), 1.85(\mathrm{~m}, 1 \mathrm{H}), 1.27$ $(\mathrm{s}, 3 \mathrm{H})$.

${ }^{13} \mathrm{C}$ NMR (126 MHz, CDCl $): \delta$ 147.0, 146.3, 144.8, 143.2, 136.9, 135.4, 134.4, 129.8, 126.9, $125.9,125.9,116.2$, 115.7, 111.6, 108.9, 108.6, 48.5, 47.2, 41.5, 39.2, 25.6, 21.5.

HRMS (ESI) $m / z[M+H]^{+}$Calcd for $\mathrm{C}_{24} \mathrm{H}_{26} \mathrm{NO}_{2} \mathrm{~S}$ 392.1679; Found 392.1676.

(5R,7S)-5-Methyl-7-(2-methylallyl)-1-tosyl-5-vinyl-1,5,6,7-tetrahydrocyclopenta[f]indole (14a) and (5R,7R)-5-Methyl-7-(2-methylallyl)-1-tosyl-5-vinyl-1,5,6,7tetrahydrocyclopenta[f]indole (14b)
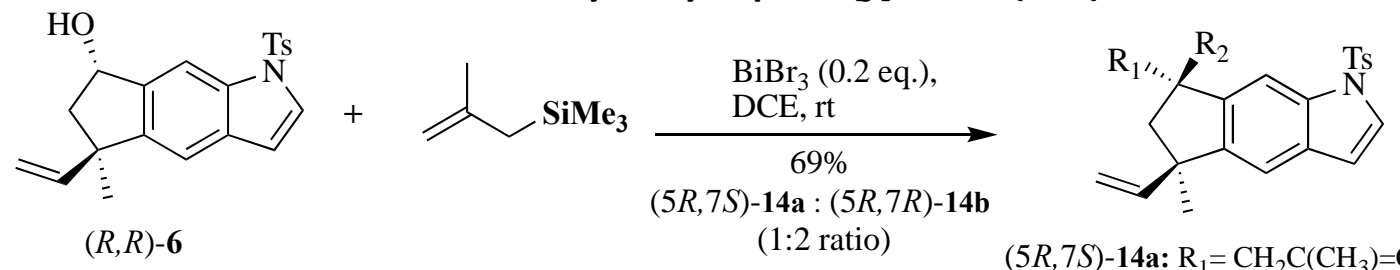

$(5 R, 7 S)-14 a: \mathrm{R}_{1}=\mathrm{CH}_{2} \mathrm{C}\left(\mathrm{CH}_{3}\right)=\mathrm{CH}_{2}$ e $\mathrm{R}_{2}=\mathrm{H}$ $(5 R, 7 R)-14 \mathbf{b}: \mathrm{R}_{1}=\mathrm{H}, \mathrm{R}_{2}=\mathrm{CH}_{2} \mathrm{C}\left(\mathrm{CH}_{3}\right)=\mathrm{CH}_{2}$

To a resealable pressure tube charged with $\mathrm{BiBr}_{3}(0.031 \mathrm{~g}, 0.069 \mathrm{mmol}, 0.2$ eq.) under nitrogen atmosphere was added a solution of indole $(R, R)-6(0.127 \mathrm{~g}, 0.345 \mathrm{mmol}, 1.0 \mathrm{eq}$.) 
and methallylsilane ${ }^{3}(0.12 \mathrm{~mL}, 0.69 \mathrm{mmol}, 2.0$ eq.) in DCE (1.7 mL). After 1 hour under stirring at room temperature the reaction mixture was concentrated under reduced pressure and the crude product was purified by flash chromatography $\left(\mathrm{SiO}_{2}, 5 \% \mathrm{EtOAc} /\right.$ hexanes) to provide a $1: 2$ mixture of $(5 R, 7 S)-14 \mathrm{a}$ and $(5 R, 7 R)-14 \mathrm{~b}$ in $69 \%$ yield $(0.096 \mathrm{~g}, 0.24 \mathrm{mmol})$ as a colorless viscous oil.

${ }^{1} \mathrm{H}$ NMR $(5 R, 7 S)-14 a$ and $\left.(5 R, 7 R)-14 b\right): \delta 7.82(\mathrm{~s}, 1 \mathrm{H}), 7.78(\mathrm{~d}, J=8.5 \mathrm{~Hz}, 2 \mathrm{H}), 7.50(\mathrm{~m}, 1 \mathrm{H})$, $7.24(\mathrm{~m}, 3 \mathrm{H}),[6.57, \mathrm{~m}$ (major) and 6.59, $\mathrm{m}$ (minor), 1H], [6.05, m (major) and $5.97 \mathrm{~m}$ (minor), $1 \mathrm{H}$ ], $5.11(\mathrm{~m}, 2 \mathrm{H}), 4.88(\mathrm{~m}, 2 \mathrm{H}),[3.50 \mathrm{~m}$ (major) and $3.38 \mathrm{~m}$ (minor), $1 \mathrm{H}], 2.70$ (dd, $J=6.0$ and $14.5 \mathrm{~Hz}, 1 \mathrm{H}), 2.35(\mathrm{~s}, 3 \mathrm{H}), 2.32(\mathrm{~m}, 1 \mathrm{H}), 2.19(\mathrm{~m}, 2 \mathrm{H}), 1.87(\mathrm{~s}, 3 \mathrm{H}), 1.28(\mathrm{~s}, 3 \mathrm{H})$.

${ }^{13} \mathrm{C}$ NMR (126 MHz, CDCl 3 ) $\delta$ (ppm): 147.1, 146.2, 146.2, 144.7, 144.3; 143.7, 135.5, 134.4, $129.8,126.9,125.9,115.6,111.8,111.6,108.9,108.6$, 48.4 , 47.6, 43.9, 39.7, 25.7, 22.5, 21.5 . HRMS (ESI) $m / z[M+H]^{+}$Calcd for $\mathrm{C}_{25} \mathrm{H}_{28} \mathrm{NO}_{2} \mathrm{~S}$ 406.1841; Found 406.1822.

IV (cm $\left.{ }^{-1}\right):$ 2964, 2914, 2857, 1634, 1595, 1494, 1441, 1370, 1340, 1285, 1230, 1183, 1167, $1120,1092,999,919,878,806,732,702,672$

(5R,7R)-5-methyl-7-(2-methylprop-1-en-1-yl)-1-tosyl-5-vinyl-1,5,6,7tetrahydrocyclopenta[f] indole (18a) and (5R,7S)-5-methyl-7-(2-methylprop-1-en-1-yl)-1tosyl-5-vinyl-1,5,6,7 tetrahydrocyclopenta[f]indole (18b)

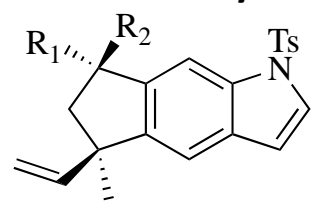

$(5 R, 7 S)-14 a: \mathrm{R}_{1}=\mathrm{CH}_{2} \mathrm{C}\left(\mathrm{CH}_{3}\right)=\mathrm{CH}_{2}$ e R $2=\mathrm{H}$ $(5 R, 7 R)-14 \mathbf{b}: \mathrm{R}_{1}=\mathrm{H}, \mathrm{R}_{2}=\mathrm{CH}_{2} \mathrm{C}\left(\mathrm{CH}_{3}\right)=\mathrm{CH}_{2}$

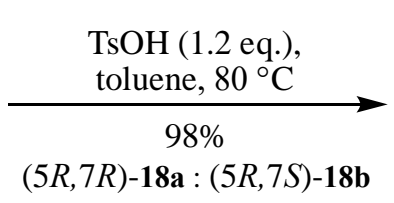
$(1: 2$ ratio)

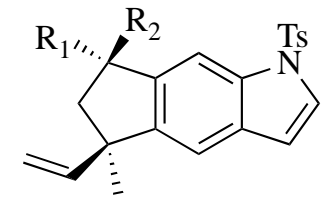

$(5 R, 7 R)-18 \mathrm{a}: \mathrm{R}_{1}=\mathrm{CH}=\mathrm{CH}\left(\mathrm{CH}_{3}\right)_{2}$ e $\mathrm{R}_{2}=\mathrm{H}$ $(5 R, 7 S)-18 b: \mathrm{R}_{1}=\mathrm{H}, \mathrm{R}_{2}=\mathrm{CH}=\mathrm{CH}\left(\mathrm{CH}_{3}\right)_{2}$

To a 1:2 mixture of indoles $14 \mathrm{a}$ and $14 \mathrm{~b}(0.0960 \mathrm{~g}, 0.237 \mathrm{mmol}, 1.0 \mathrm{eq}$.$) in a resealable$ pressure tube was added dry toluene $(8 \mathrm{~mL})$. Then $\mathrm{TsOH}(0.050 \mathrm{~g}, 0.28 \mathrm{mmol}, 1.2$ eq.) was added and the temperature was increased to $80{ }^{\circ} \mathrm{C}$ using an oil bath. The reaction progress was accompanied by ${ }^{1} \mathrm{H} N M R$ and after $4 \mathrm{~h}$, the reaction mixture was cooled to $\mathrm{rt}$ and concentrated under reduced pressure. The crude product was treated with satd. aq. $\mathrm{NaHCO}_{3}$ soln. $(10 \mathrm{~mL})$ and extracted with EtOAc $(3 \times 15 \mathrm{~mL})$. The mixture of products $(5 R, 7 R)-18 \mathrm{a}$ and $(5 R, 7 \mathrm{~S})$-18b was obtained as a brown viscous oil in $98 \%$ yield $(0.0945 \mathrm{~g}, 0.233 \mathrm{mmol})$ and used in the next step without purification. 
${ }^{1} \mathrm{H}$ NMR ((5R,7R)-18a and (5R,7S)-18b): $\delta 7.75(\mathrm{~m}, 3 \mathrm{H}), 7.60(\mathrm{~s}, 1 \mathrm{H}), 7.48(\mathrm{~d}, J=4.0 \mathrm{~Hz}, 1 \mathrm{H})$, $7.22(\mathrm{~m}, 2 \mathrm{H}), 7.14(\mathrm{~s}, 1 \mathrm{H}),[6.59 \mathrm{~m}$ (minor) and $6.56 \mathrm{~m}$ (major), 1H], [6.05 m (major) and 6.00 m (minor), 1H], $5.17(\mathrm{~m}, 1 \mathrm{H}), 5.11(\mathrm{~m}, 1 \mathrm{H}), 4.15 \mathrm{~m}$ (major) and $4.02 \mathrm{~m}$ (minor), 1H], 2.35 (s, $3 \mathrm{H}), 2.16(\mathrm{~m}, 1 \mathrm{H}), 1.86(\mathrm{~m}, 1 \mathrm{H}), 1.86(\mathrm{~s}, 6 \mathrm{H}), 1.30(\mathrm{~s}, 3 \mathrm{H})$.

${ }^{13} \mathrm{C}$ NMR (126 MHz, CDCl 3 ) $\delta$ (ppm): 146.6, 146.1, 144.7, 143.6, 135.4, 134.4, 133.1, 130.1, 129.7, 127.7, 127.0, 125.8, 115.4, 111.7, 109.3, 108.9, 48.9, 48.7, 41.5, 25.9, 24.9, 21.5, 18.3. IV $\left(\mathrm{cm}^{-1}\right)$ : 2961, 2923, 2860, 1639, 1598, 1458, 1439, 1367, 1342, 1290, 1238, 1189, 1169, $1114,1032,996,908,883,883,809,760,732,699,669$.

\section{6-lodo-1-tosil-1H-indol (17)}
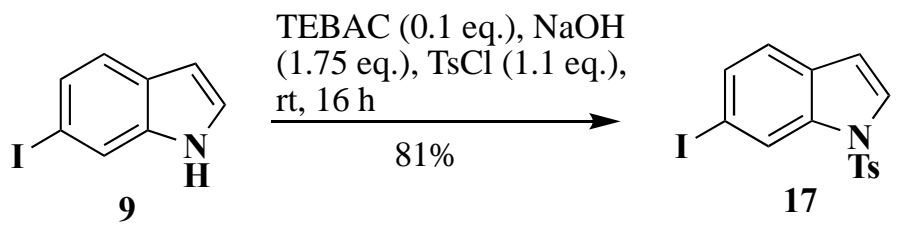

To a solution of indole 9 (0.854 g, $3.51 \mathrm{mmol}, 1.00$ eq.) in DCM (35 mL) was added TEBAC ( $0.080 \mathrm{~g}, 0.35 \mathrm{mmol}, 0.10$ eq.). Then, $\mathrm{NaOH}(0.203 \mathrm{~g}, 6.15 \mathrm{mmol}, 1.75$ eq.) and $\mathrm{TsCl}(0.734 \mathrm{~g}$, $3.864 \mathrm{mmol}, 1.10$ eq.) were added. The reaction mixture was stirred at room temperature for $16 \mathrm{~h}$ and quenched with $\mathrm{H}_{2} \mathrm{O}(50 \mathrm{~mL})$ and of $1 \mathrm{M} \mathrm{HCl}(30 \mathrm{~mL})$. After stirring for $10 \mathrm{~min}$, the aqueous layer was separated and extracted with DCM (3 x $100 \mathrm{~mL})$. The organic layer was washed with brine, dried over $\mathrm{MgSO}_{4}$ and evaporated under reduced pressure. The resulting residue was purified by flash column chromatography $\left(\mathrm{SiO}_{2}, 5-10 \% \mathrm{AcOEt} / \mathrm{hexanes}\right)$ to provide $17(1,14 \mathrm{~g}, 2,87 \mathrm{mmol})$ in $82 \%$ yield as a yellow solid.

${ }^{1} \mathrm{H}$ NMR (300 MHz, CDCl 3$): \delta 8.36(\mathrm{t}, J=6,6 \mathrm{~Hz}, 1 \mathrm{H}), 7.75(\mathrm{~d}, \mathrm{~J}=8.4 \mathrm{~Hz}, 2 \mathrm{H}), 7.52-7.47(\mathrm{~m}, 2$ H), $7.27-7.22(\mathrm{~m}, 3 \mathrm{H}), 6.61(\mathrm{dd}, J=3.9$ and $0.9 \mathrm{~Hz}, 1 \mathrm{H}), 2.34(\mathrm{~s}, 3 \mathrm{H})$.

${ }^{13} \mathrm{C}$ NMR (75 MHz, $\left.\mathrm{CDCl}_{3}\right): \delta 145.2,135.7,135.0,132.2,130.0,129.9,126.7,126.5,122.8$, $122.3,108.7,88.7,21.5$.

HRMS (ESI) $m / z[M+H]^{+}$Calcd for $\mathrm{C}_{15} \mathrm{H}_{13} \mathrm{INO}_{2} \mathrm{~S} 397.9707$; Found 397.9708 .

$\mathrm{Mp}=124.8-125.3^{\circ} \mathrm{C}$. 
(5R,7R)-5-Methyl-1-tosyl-5-((E)-2-(1-tosyl-1H-indol-6-yl)vinyl)-1,5,6,7tetrahydrocyclopenta[f]indol-7-ol (15)<smiles></smiles>

$(+/-)-6$<smiles>[Y]n1ccc2ccc(I)cc21</smiles>

17

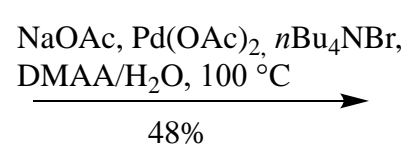

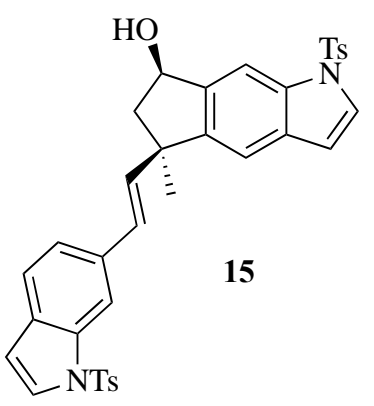

To a dried round bottom flask charged with the tricyclic indole (+/-)-6 $(0.030 \mathrm{~g}, 0.082 \mathrm{mmol}$, 1.00 eq.), $N$-tosyl 6-iodo-indole 17 (0.065 g, 0.16 mmol; 2.0 eq.), NaOAc (0.013 g, 0.16 mmol, 2.0 eq.), $\mathrm{Pd}(\mathrm{OAc})_{2}(0.002 \mathrm{~g}, 0,008 \mathrm{mmol}, 0.1$ eq.) and $n-\operatorname{Bu} 4 \mathrm{NBr}(0.0060 \mathrm{~g}, 0.016 \mathrm{mmol}, 0.20$ eq.). Degassed DMAA/ $\mathrm{H}_{2} \mathrm{O}(0.96 \mathrm{~mL}, 9: 1, \mathrm{v} / \mathrm{v})$ was added under a nitrogen atmosphere and the reaction mixture was kept under stirring at $100{ }^{\circ} \mathrm{C}$ using an oil bath for $12 \mathrm{~h}$. After that, the reaction mixture was allowed to reach $\mathrm{rt}$ and quenched by the addition of $\mathrm{H}_{2} \mathrm{O}(2 \mathrm{~mL})$, extracted with DCM $(3 \times 5 \mathrm{~mL})$, washed with saturated solution of $\mathrm{NaHCO}_{3}(30 \mathrm{~mL})$ and brine (40 mL). The combined organics were dried over $\mathrm{MgSO}_{4}$, filtered and the solvent was removed by reduced pressure. The crude product was purified by flash column chromatography $\left(\mathrm{SiO}_{2}\right.$, $5-10 \%$ AcOEt/hexanes) to furnish tosyl indole 15 ( $0.025 \mathrm{~g}, 0.039 \mathrm{mmol})$ in $48 \%$ yield as a yellow oil.

${ }^{1} \mathrm{H}$ NMR (500 MHz, CDCl $): \delta 8.05(\mathrm{~m}, 1 \mathrm{H}), 7.91(\mathrm{~m}, 1 \mathrm{H}), 7.82(\mathrm{~m}, 2 \mathrm{H}), 7.73(\mathrm{~m}, 2 \mathrm{H}), 7.59(\mathrm{~d}, \mathrm{~J}$ $=3.5 \mathrm{~Hz}, 1 \mathrm{H}), 7.49(\mathrm{~d}, \mathrm{~J}=4.0 \mathrm{~Hz}, 1 \mathrm{H}), 7.41(\mathrm{~m}, 1 \mathrm{H}), 7.32(\mathrm{~s}, 1 \mathrm{H}), 7.29(\mathrm{dd}, \mathrm{J}=1.5$ and $8.0 \mathrm{~Hz}$, 1H), $7.25(\mathrm{~m}, 2 \mathrm{H}), 7.21(\mathrm{~m}, 2 \mathrm{H}), 6.63(\mathrm{dd}, \mathrm{J}=0.8$ and $3.6 \mathrm{~Hz}, 1 \mathrm{H}), 6.59(\mathrm{dd}, \mathrm{J}=0.65$ and 3.65 $\mathrm{Hz}, 1 \mathrm{H}), 6.56(\mathrm{~d}, \mathrm{~J}=16.1 \mathrm{~Hz}, 1 \mathrm{H}), 6.49(\mathrm{~d}, \mathrm{~J}=16.1 \mathrm{~Hz}, 1 \mathrm{H}), 5.38(\mathrm{t}, \mathrm{J}=6.2 \mathrm{~Hz}, 1 \mathrm{H}), 2.55(\mathrm{~m}, 1 \mathrm{H})$, $2.35(\mathrm{~s}, 3 \mathrm{H}), 2.33(\mathrm{~s}, 3 \mathrm{H}), 2.26(\mathrm{~m}, 1 \mathrm{H}), 1.47(\mathrm{~s}, 3 \mathrm{H})$.

${ }^{13}$ C NMR (126 MHz, CDCl $): \delta 144,9,144,9,141,7,139.0,135,4,135,4,135.4,134.6,134.2$, $131.8,130.0,130.0,127.4,126.9,126.7,126.6$, 121.4, 121.3, 116.3, 111.7, 109.5, 109.1, $108.8,75.0,74.5,51.7,47.5,27.0,24.8,21.6,21.5$.

HRMS (ESI-TOF) $m / z\left[\mathrm{M}+\mathrm{H}-\mathrm{H}_{2} \mathrm{O}\right]^{+}$Calcd for $\mathrm{C}_{36} \mathrm{H}_{31} \mathrm{~N}_{2} \mathrm{O}_{4} \mathrm{~S}_{2}$ 619.1720; Found 619.1710. 
(5R,7R)-5-methyl-7-(2-methylprop-1-en-1-yl)-1-tosyl-5-((E)-2-(1-tosyl-1H-indol-6-yl)vinyl)-

1,5,6,7-tetrahydrocyclopenta[f]indole (19a) and

(5R,7S)-5-methyl-7-(2-methylprop-1-en-1-yl)-1-tosyl-5-((E)-2-(1-tosyl-1H-indol-6-yl)vinyl)1,5,6,7-tetrahydrocyclopenta[f]indole (19b)

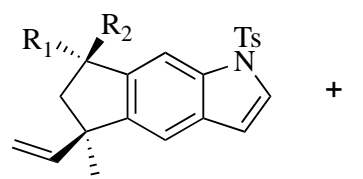

$(5 R, 7 R)-18 \mathrm{a}: \mathrm{R}_{1}=\mathrm{CH}=\mathrm{CH}\left(\mathrm{CH}_{3}\right)_{2}$ e $\mathrm{R}_{2}=\mathrm{H}$ $(5 R, 7 S)-18 b: \mathrm{R}_{1}=\mathrm{H}, \mathrm{R}_{2}=\mathrm{CH}=\mathrm{CH}\left(\mathrm{CH}_{3}\right)_{2}$

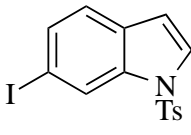

17

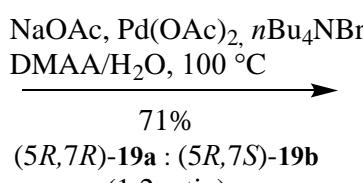
$(1: 2$ ratio)

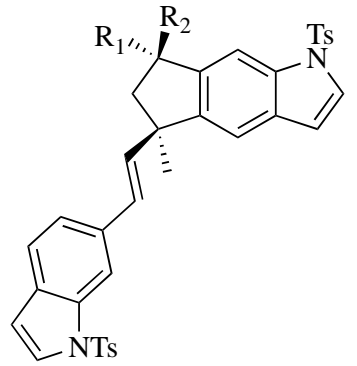

$(5 R, 7 R)-19 \mathrm{a}: \mathrm{R}_{1}=\mathrm{CH}=\mathrm{CH}\left(\mathrm{CH}_{3}\right)_{2}$ e $\mathrm{R}_{2}=\mathrm{H}$ $(5 R, 7 S)-19 b: \mathrm{R}_{1}=\mathrm{H}, \mathrm{R}_{2}=\mathrm{CH}=\mathrm{CH}\left(\mathrm{CH}_{3}\right)_{2}$

To a dried round bottom flask charged with a 1:2 mixture of tricyclic indoles $\mathbf{1 8 a}$ and $\mathbf{1 8 b}$ (0.0080 g, 0.020 mmol, 1.0 eq.), iodoindole 17 (0.016 g, 0.039 mmol; 2.0 eq.), NaOAc (0.0030 g, $0.039 \mathrm{mmol}, 2.0$ eq.), $\mathrm{Pd}(\mathrm{OAc})_{2}(0.044 \mathrm{~g}, 0,0020 \mathrm{mmol}, 0.10$ eq.) and $n-\mathrm{Bu} 4 \mathrm{NBr}(0.001 \mathrm{~g}$, $0.004 \mathrm{mmol}, 0.2$ eq.) was added degassed $\mathrm{DMAA} / \mathrm{H}_{2} \mathrm{O}(0.5 \mathrm{~mL}, 9: 1, \mathrm{v} / \mathrm{v})$ under nitrogen atmosphere and the reaction mixture was kept under stirring at $100{ }^{\circ} \mathrm{C}$ using an oil bath for $22 \mathrm{~h}$. After that, the reaction mixture was allowed to reach $\mathrm{rt}$ and quenched with $\mathrm{H}_{2} \mathrm{O}(1 \mathrm{~mL})$ extracted with DCM $(3 \times 5 \mathrm{~mL})$, washed with saturated solution of $\mathrm{NaHCO}_{3}(15 \mathrm{~mL})$ and brine $(20 \mathrm{~mL})$, dried over $\mathrm{MgSO}_{4}$, filtered and the solvent was removed under reduced pressure. The crude product was purified by flash column chromatography $\left(\mathrm{SiO}_{2}, 5-10 \% \mathrm{AcOEt} /\right.$ hexanes) to furnish a mixture of diastereomers $(5 R, 7 R)-19 \mathrm{a}$ and $(5 R, 7 S)-19 \mathrm{~b}(0.0096 \mathrm{~g}, 0.014 \mathrm{mmol})$, in $71 \%$ yield, as a yellow solid.

The diastereoisomers were separated by preparative HPLC using a chiral column Chiralpak ${ }^{\circledR}$ IA (21 x $250 \mathrm{~mm}, 5 \mu \mathrm{m}, 3: 97 \mathrm{v} / \mathrm{v}, i-\mathrm{PrOH} / \mathrm{h}$ exanes, isocratic mode, flow $12.6 \mathrm{~mL} / \mathrm{min}, \mathrm{UV}$ detection at $291 \mathrm{~nm})$.

Minor isomer $(5 R, 7 R)-19 \mathrm{a}$

${ }^{1}{ }_{\mathrm{H}} \mathrm{NMR}\left(400 \mathrm{MHz}, \mathrm{CDCl}_{3}\right): \delta 7.83(\mathrm{~s}, 1 \mathrm{H}), 7.79(\mathrm{~d}, J=8.4 \mathrm{~Hz}, 2 \mathrm{H}), 7.72(\mathrm{~d}, \mathrm{~J}=8.4 \mathrm{~Hz}, 2 \mathrm{H}$ ), 7.65 (s, 1H), $7.54(\mathrm{~d}, J=3.6 \mathrm{~Hz}, 1 \mathrm{H}), 7.47(\mathrm{~d}, J=3.6 \mathrm{~Hz}, 1 \mathrm{H}), 7.38(\mathrm{~d}, J=8.4 \mathrm{~Hz}, 1 \mathrm{H}), 7.31(\mathrm{~s}, 1 \mathrm{H})$, $7.23(\mathrm{~m}, 5 \mathrm{H}), 6.64(\mathrm{dd}, J=0.8$ and $3.6 \mathrm{~Hz}, 1 \mathrm{H}), 6.57(\mathrm{dd}, J=0.8$ and $3.6 \mathrm{~Hz}, 1 \mathrm{H}), 6.43(\mathrm{~d}, J=$ $16.0 \mathrm{~Hz}, 1 \mathrm{H}), 6.10(\mathrm{~d}, J=16.0 \mathrm{~Hz}, 1 \mathrm{H}), 5.18(\mathrm{~m}, 1 \mathrm{H}), 4.09(\mathrm{~m}, 1 \mathrm{H}), 2.47(\mathrm{~m}, 1 \mathrm{H}), 2.35(\mathrm{~d}, J=10.4$ $\mathrm{Hz}, 6 \mathrm{H}), 1.88(\mathrm{~s}, 3 \mathrm{H}), 1.84(\mathrm{~s}, 3 \mathrm{H}), 1.84(\mathrm{~m}, 1 \mathrm{H}), 1.25(\mathrm{~s}, 3 \mathrm{H})$. 
${ }^{13} \mathrm{C}$ NMR (126 MHz, CDCl 3$): \delta$ 144.9, 144.8, 144.7, 144.4, 138.1, 135.4, 135.4, 135.4, 134.5, $134.5,133.4,130.1,129.9,129.8,129.7,127.7,127.0,126.7,126.6,126.4,125.8,121.5$, 121.1, 115.8, 111.5, 109.2, 109.1, 109.0, 49.6, 48.5, 41.2, 26.9, 25.9, 21.6, 21.5, 18.4.

IV (cm-1): 3896, 3849, 3838, 3747, 3742, 3497, 3494, 3472, 1450, 1433, 1367, 1169, 1117, $1090,996,881,809,732,696.6$.

HRMS (ESI) $m / z[M+H]^{+}$of 19a and 19b Calcd for $\mathrm{C}_{40} \mathrm{H}_{39} \mathrm{~N}_{2} \mathrm{O}_{4} \mathrm{~S}_{2}$ 675.2351; Found 675.2313.

Mp: $106.7-109.1^{\circ} \mathrm{C}$.

Major isomer (5R,7S)-19b

${ }^{1} \mathrm{H}$ NMR (400 MHz, $\left.\mathrm{CDCl}_{3}\right): \delta 7.96(\mathrm{~s}, 1 \mathrm{H}), 7.76(\mathrm{~m}, 4 \mathrm{H}), 7.65(\mathrm{~s}, 1 \mathrm{H}), 7.50(\mathrm{~m}, 2 \mathrm{H}), 7.43(\mathrm{~d}, \mathrm{~J}=$ $8.0 \mathrm{~Hz}, 1 \mathrm{H}), 7.34(\mathrm{dd}, \mathrm{J}=1.6,8.4 \mathrm{~Hz}, 1 \mathrm{H}), 7.22(\mathrm{~m}, 5 \mathrm{H}), 6.65(\mathrm{~d}, \mathrm{~J}=16.0,1 \mathrm{H}), 6.60$ (dd, J = 0.8, $3.6 \mathrm{~Hz}, 1 \mathrm{H}), 6.56(\mathrm{dd}, \mathrm{J}=0.8,3.6 \mathrm{~Hz}, 1 \mathrm{H}), 6.49(\mathrm{~d}, \mathrm{~J}=16,1 \mathrm{H}), 5.22(\mathrm{~m}, 1 \mathrm{H}), 4.23(\mathrm{~m}, 1 \mathrm{H}), 2.36$ (d, J = 9.6 Hz, 6H), $2.30(m, 1 H), 2.00(m, 1 H), 1.89(d, J=1.2 \mathrm{~Hz}, 6 \mathrm{H}), 1.45(\mathrm{~s}, 3 \mathrm{H})$.

${ }^{13} \mathrm{C}$ NMR (126 MHz, CDCl 3$): \delta$ 146.2, 144.9, 144.8, 143.6, 143.5, 138.6, 135.4, 135.4, 134.6, $134.5,133.3,130.2$, 129.9, 129.9, 127.6, 127.4, 127.0, 126.7, 126.5, 125.9, 121.4, 121.3, 114.4, 115.6, 111.6, 109.4, 109.1, 108.9, 49.4, 48.5, 41.6, 25.9, 25.6, 21.6, 21.5, 18.4.

HRMS (ESI) $m / 2[\mathrm{M}+\mathrm{H}]^{+}$of 19a and 19b Calcd for $\mathrm{C}_{40} \mathrm{H}_{39} \mathrm{~N}_{2} \mathrm{O}_{4} \mathrm{~S}_{2}$ 675.2351; Found 675.2313.

Mp: $104.2-109.2^{\circ} \mathrm{C}$.

IV (cm $\left.{ }^{-1}\right): 3846,3830,3813,3791,1436,1373,1271,1172,1114,1092,1026,1002,966,848$, $812,719,699$.

(5R,7R)-5-((E)-2-(1H-Indol-6-yl)vinyl)-5-methyl-7-(2-methylprop-1-en-1-yl)-1,5,6,7tetrahydrocyclopenta[f]indole (1)

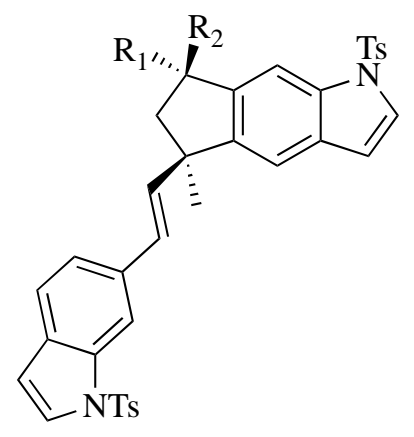

$(5 R, 7 R)-19 \mathrm{a}: \mathrm{R}_{1}=\mathrm{CH}=\mathrm{CH}\left(\mathrm{CH}_{3}\right)_{2}$ e $\mathrm{R}_{2}=\mathrm{H}$ $(5 R, 7 S)-19 b: \mathrm{R}_{1}=\mathrm{H}, \mathrm{R}_{2}=\mathrm{CH}=\mathrm{CH}\left(\mathrm{CH}_{3}\right)_{2}$
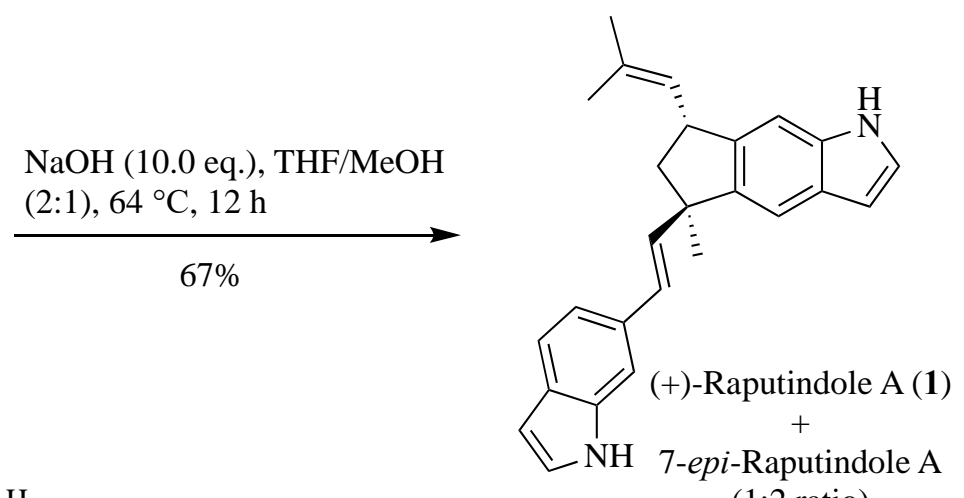

(1:2 ratio) 
To a solution of bisindole mixture 19a:19b (0.055 $\mathrm{g}, 0.081 \mathrm{mmol}, 1.0 \mathrm{eq}$.) dissolved in a mixture of $\mathrm{MeOH} / \mathrm{THF}(1.2 \mathrm{~mL}, 2: 1, \mathrm{v} / \mathrm{v})$ was added $\mathrm{NaOH}(0.036 \mathrm{~g}, 0.81 \mathrm{mmol}, 10$ eq.). The reaction mixture was warmed to $64{ }^{\circ} \mathrm{C}$ using a heating block and stirred during $12 \mathrm{~h}$. Upon completion of the reaction, the organic solvent was removed under reduced pressure and $\mathrm{H}_{2} \mathrm{O}$ was added $(1 \mathrm{~mL})$. The mixture was extracted with EtOAc $(3 \times 3 \mathrm{~mL})$ and the combined organic layer was washed with brine $(10 \mathrm{~mL})$. After drying over $\mathrm{MgSO}_{4}$, the solvent was removed under reduced pressure and the residue was purified by column chromatography (activated neutral alumina, 5-20\% EtOAc/hexanes) to provide raputindole $\mathrm{A}(\mathbf{1})$, as the minor isomer, together with its C- 6 epimer ( $1: 2$ ratio), in $67 \%$ yield $(0.020 \mathrm{~g}, 0.055 \mathrm{mmol})$ as a yellow oil. The diastereoisomers were separated by preparative HPLC using a chiral column Chiralpak ${ }^{\circledR}$ IA $(21 \times 250 \mathrm{~mm}, 5 \mu \mathrm{m}, 12: 88 \mathrm{v} / \mathrm{v}, i-\mathrm{PrOH} /$ hexane, isocratic mode, flow 12.6 $\mathrm{mL} / \mathrm{min}, \mathrm{UV}$ detection at $291 \mathrm{~nm})$. Pure (+)-raputindole $\mathrm{A}(\mathbf{1})\left\{[\alpha]^{22} \mathrm{D}=+76(c 0.10, \mathrm{MeOH}, 20\right.$ $\left.\left.{ }^{\circ} \mathrm{C}\right)\right\}$ was isolated $\left(\mathrm{R}_{\mathrm{T}}=32.11 \mathrm{~min}\right)$ and its $\mathrm{C}-6$ epimer $\left(\mathrm{R}_{\mathrm{T}}=36.73 \mathrm{~min}\right)$ both as colorless oil.

\section{(+)-Raputindole A (1)}

${ }^{1} \mathrm{H}$ NMR $\left(500 \mathrm{MHz}^{\mathrm{CDCl}} \mathrm{CD}_{3}\right) \delta(\mathrm{ppm})=8.04\left(\mathrm{br} \mathrm{s}, 1 \mathrm{H}, \mathrm{H}-1^{\prime}\right), 8.02\left(\mathrm{br} \mathrm{s}, 1 \mathrm{H}, \mathrm{H}-1^{\prime \prime}\right), 7.50$ (d, $J=$ 8.25, 1H, H-4"), $7.44\left(\mathrm{~s}, 1 \mathrm{H}, \mathrm{H}-4^{\prime}\right), 7,26\left(\mathrm{~s}, 1 \mathrm{H}, \mathrm{H}-\mathrm{7}^{\prime \prime}\right), 7.17$ (t, J=2.45, 1H, H-2'), $7.15(\mathrm{~m}, 1 \mathrm{H}, \mathrm{H}-$ 5"), $7.13\left(\mathrm{~m}, 1 \mathrm{H}, \mathrm{H}-2^{\prime \prime}\right), 7.08\left(\mathrm{~s}, 1 \mathrm{H}, \mathrm{H}-\mathrm{7}^{\prime}\right), 6.53\left(\mathrm{~m}, 1 \mathrm{H}, \mathrm{H}-3^{\prime}\right), 6.47\left(\mathrm{~m}, 1 \mathrm{H}, \mathrm{H}-3^{\prime \prime}\right), 6.45$ (d, J = 15.9, $1 \mathrm{H}, \mathrm{H}-2$ ), 6.15 (d, J = 16.9, 1H, H-1), 5.22 (d, J = 9.0, 1H, H-7), 4.07 (m, 1H, H-6), 2.44 (dd, $J=6.85$ and 12.1, $1 \mathrm{H}, \mathrm{H}-5 \beta), 1.82(\mathrm{~m}, 1 \mathrm{H}, \mathrm{H}-5 \alpha), 1.82(\mathrm{~d}, 3 \mathrm{H}, J=1.4, \mathrm{H}-9), 1.79(\mathrm{~d}, 3 \mathrm{H}, J=1.3$, $\mathrm{H}-10), 1.61$ (s, 3H, H-4).

${ }^{13} \mathrm{C}$ NMR (800 MHz, CDCl 3 ) $\delta(\mathrm{ppm})=142.2\left(\mathrm{C}-6^{\prime}\right), 141.5$ (C-5'), 137.2 (C-2), 136.2 (C-7"a), 135.7 (C-7'a), 132.6 (C-8), 132.2 (C-6"), 128.3 (C-7), 127.2 (C-3'a), 127.0 (C-3"a), 126.8 (C-1), 124.3 (C-2"), 123.7 (C-2'), 120,5 (C-4"), 118.5 (C-5"), 115,0 (C-4'), 108.9 (C-7"), 106.2 (C-7'), 102.6 (C-3"), 102.5 (C-3'), 49.9 (C-5), 48.4 (C-3), 41.0 (C-6), 27.2 (C-4), 25.9 (C-9), 18.3 (C-10). HRMS (ESI) $\mathbf{m} / \mathbf{z}\left[\mathrm{M}+\mathrm{H}^{+}\right]$Calcd for $\mathrm{C}_{26} \mathrm{H}_{27} \mathrm{~N}_{2}$ 367.2174; Found 367.2166.

$[\alpha]^{22} \mathrm{D}=+76\left(\mathrm{c} 0.10, \mathrm{MeOH}, 20^{\circ} \mathrm{C}\right)$ 
DAD1 G, Sig=291,4 Ref=360,100 (JulianalJR652_88 12 hex isop.D)

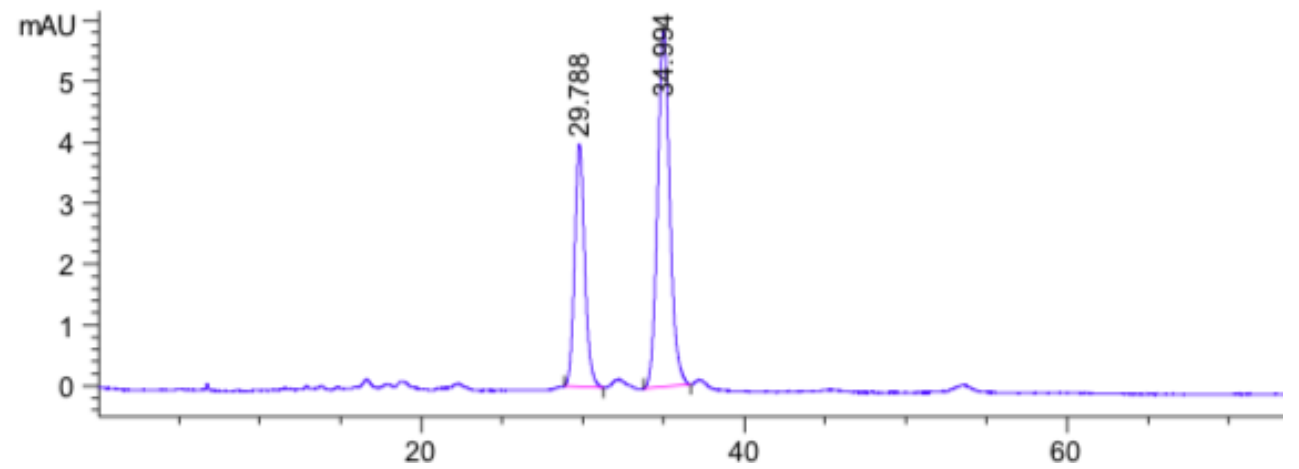

(JulianaUR652_88 12 hex isop.D)

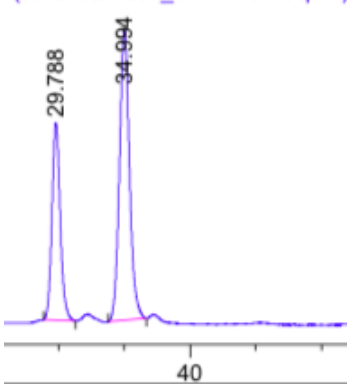

\begin{tabular}{|c|c|c|c|c|c|c|}
\hline $\begin{array}{c}\text { Peak } \\
\#\end{array}$ & $\begin{array}{l}\text { Ret Ti me } \\
{[\mathrm{mi} \mathrm{n}]}\end{array}$ & Type & $\begin{array}{l}W d t h \\
{[m n]}\end{array}$ & $\begin{array}{c}\text { Area } \\
\text { [ } \mathrm{mAU}^{*} \mathrm{~s} \text { ] }\end{array}$ & $\begin{array}{l}\text { Hei ght } \\
\text { [ } \mathrm{mAU}]\end{array}$ & $\begin{array}{c}\text { Ar ea } \\
\%\end{array}$ \\
\hline & 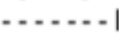 & & . & 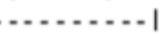 & $\ldots . .$. & $\ldots$. \\
\hline 1 & 5. 101 & BB & 0. 1334 & 39. 54308 & 4. 54919 & 4. 3045 \\
\hline 2 & 5. 559 & $\mathrm{BB}$ & 0.1065 & 8. 09878 & 1. 17390 & 0.8816 \\
\hline 3 & 6. 742 & BB & 0.1252 & 12. 59813 & 1. 51287 & 1. 3714 \\
\hline 4 & 29. 789 & $\mathrm{BB}$ & 0.6465 & 314. 62854 & 7. 24699 & 34. 2493 \\
\hline 5 & 34. 993 & BB & 0. 7939 & 543. 77362 & 10. 26778 & 59. 1932 \\
\hline
\end{tabular}

Figure 3. HPLC chromatogram of mixture (+)-raputindole A (1) and 7-epi-raputindole A. Chiral column Chiralpak® IA $(4.6 \times 250 \mathrm{~mm}, 5 \mu \mathrm{m}, 12: 88 \mathrm{v} / \mathrm{v}, i-\mathrm{PrOH} /$ hexane, isocratic mode, flow $0.6 \mathrm{~mL} / \mathrm{min}$, UV detection at $291 \mathrm{~nm}$ ).

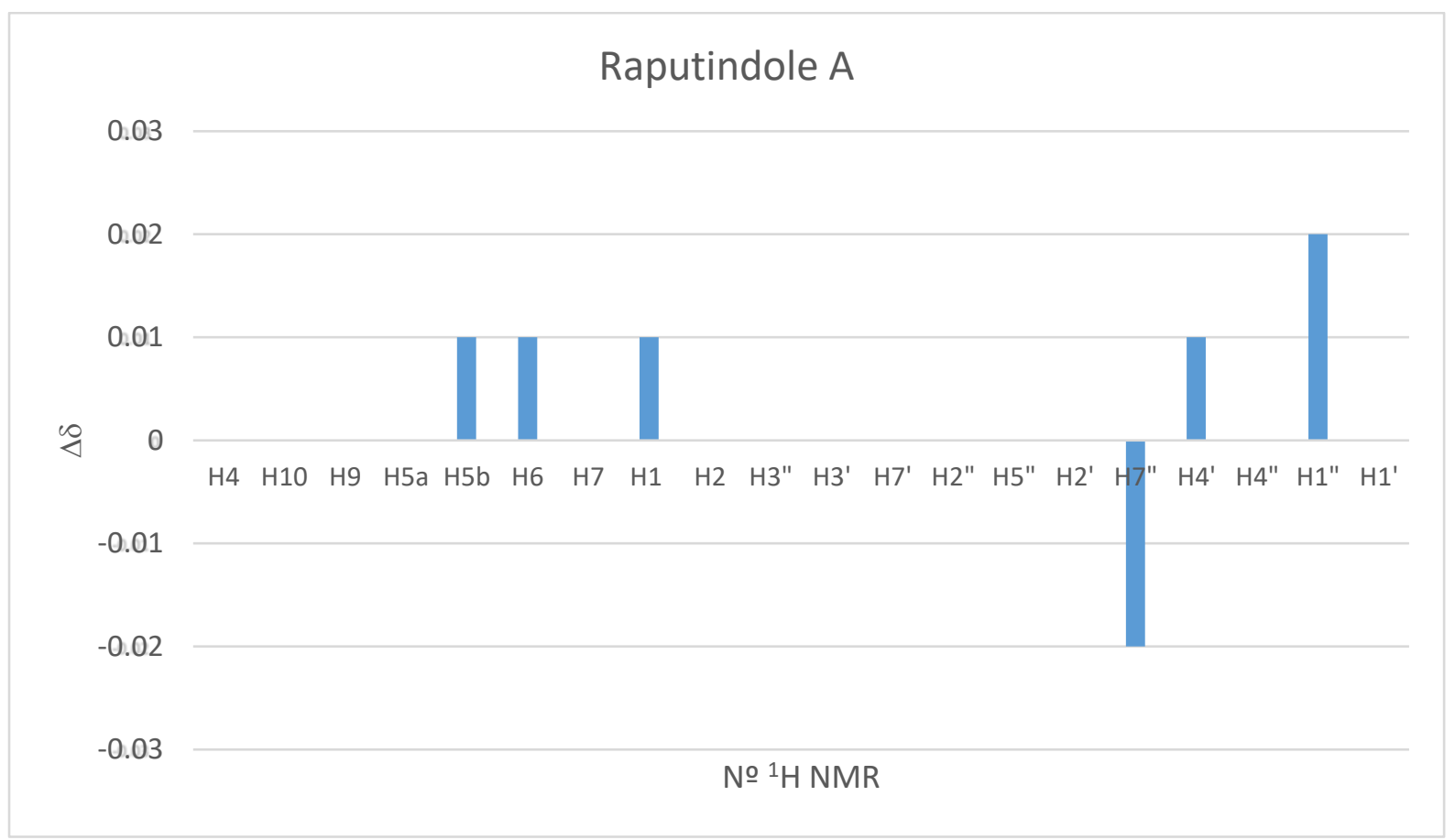

Figure 4. Differences $\delta(\mathrm{ppm})$ between ${ }^{1} \mathrm{H}$ NMR (+)-raputindole $\mathrm{A}(\mathbf{1})$ synthetic and natural. 


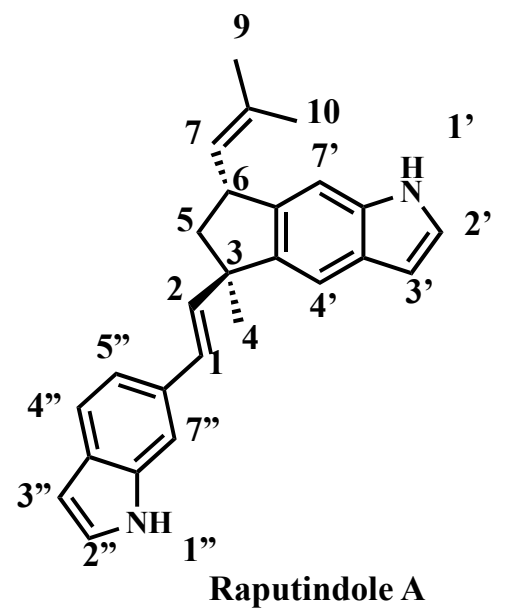

Figure 5. Numbering of Hydrogens.

Table 2. Comparison of ${ }^{1} \mathrm{H}$ NMR chemical shifts of synthetic and natural (+)-raputindole $A(\mathbf{1})$, in ppm.

\begin{tabular}{|c|c|c|c|}
\hline Hydrogen & Natural & JR670 & (Nat - JR670) \\
\hline $\mathrm{H} 4$ & 1,61 & 1,61 & 0 \\
\hline $\mathrm{H} 10$ & 1,79 & 1,79 & 0 \\
\hline H9 & 1,82 & 1,82 & 0 \\
\hline $\mathrm{H} 5 \mathrm{a}$ & 1,82 & 1,82 & 0 \\
\hline $\mathrm{H} 5 \mathrm{~b}$ & 2,45 & 2,44 & 0,01 \\
\hline H6 & 4,08 & 4,07 & 0,01 \\
\hline $\mathrm{H} 7$ & 5,22 & 5,22 & 0 \\
\hline $\mathrm{H} 1$ & 6,14 & 6,15 & 0,01 \\
\hline $\mathrm{H} 2$ & 6,45 & 6,45 & 0 \\
\hline H3" & 6,47 & 6,47 & 0 \\
\hline H3' & 6,53 & 6,53 & 0 \\
\hline $\mathrm{H} 7^{\prime}$ & 7,08 & 7,08 & 0 \\
\hline H2" & 7,13 & 7,13 & 0 \\
\hline H5" & 7,15 & 7,15 & 0 \\
\hline $\mathrm{H} 2^{\prime}$ & 7,17 & 7,17 & 0 \\
\hline H7" & 7,24 & 7,26 & $-0,02$ \\
\hline H4 & 7,45 & 7,44 & 0,01 \\
\hline H4" & 7,5 & 7,5 & 0 \\
\hline
\end{tabular}




$\begin{array}{llll}H 1^{\prime \prime} & 8 & 8,02 & 0,02 \\ H 1^{\prime} & 8,04 & 8,04 & 0\end{array}$

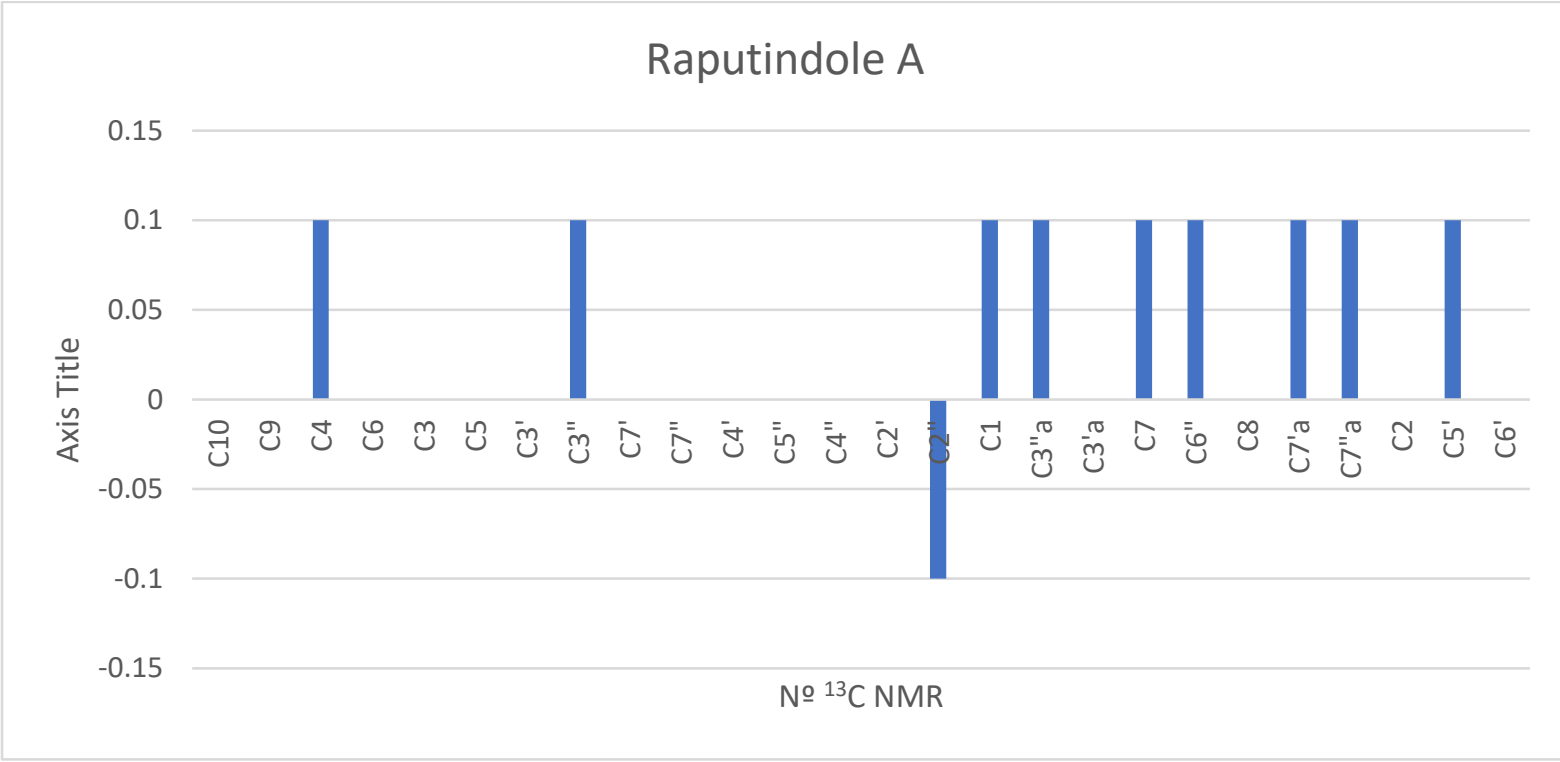

Figure 5. Differences $\delta(\mathrm{ppm})$ between ${ }^{13} \mathrm{C}$ NMR (+)-raputindole $\mathrm{A}(\mathbf{1})$ synthetic and natural.

Table 3. Comparison of ${ }^{13} \mathrm{C}$ NMR chemical shifts of (+)-raputindole $A(1)$ synthetic and natural in ppm.

\begin{tabular}{llll}
\hline Carbon & Natural & JR670 & (Nat - JR670) \\
C10 & 18,3 & 18,3 & 0 \\
C9 & 25,9 & 25,9 & 0 \\
C4 & 27,20 & 27,1 & 0,1 \\
C6 & 41 & 41 & 0 \\
C3 & 48,4 & 48,4 & 0 \\
C5 & 50 & 50 & 0 \\
C3' & 102,6 & 102,5 & 0 \\
C3" & 102,7 & 102,6 & 0,1 \\
C7' & 106,2 & 106,2 & 0 \\
C7" & 108,9 & 108,9 & 0 \\
C4' & 115 & 115 & 0 \\
C5" & 118,5 & 118,5 & 0
\end{tabular}




\begin{tabular}{llll} 
C4" & 120,5 & 120,5 & 0 \\
C2' & 123,7 & 123,7 & 0 \\
C2" & 124,2 & 124,3 & $-0,1$ \\
C1 & 126,9 & 126,8 & 0,1 \\
C3"a & 127,1 & 127 & 0,1 \\
C3'a & 127,2 & 127,2 & 0 \\
C7 & 128,4 & 128,3 & 0,1 \\
C6" & 132,3 & 132,2 & 0,1 \\
C8 & 132,6 & 132,6 & 0 \\
C7'a & 135,8 & 135,7 & 0,1 \\
C7"a & 136,3 & 136,2 & 0,1 \\
C2 & 137,2 & 137,2 & 0 \\
C5' & 141,6 & 141,5 & 0,1 \\
C6' & 142,2 & 142,2 & 0 \\
\hline
\end{tabular}

7-epi-Raputindole A (7-epi-1)

${ }^{1} \mathrm{H}$ NMR $\left(500 \mathrm{MHz}, \mathrm{CDCl}_{3}\right) \delta(\mathrm{ppm})=8.10(\mathrm{brs}, 1 \mathrm{H}), 8.02(\mathrm{brs}, 1 \mathrm{H}), 7.56(\mathrm{~d}, \mathrm{~J}=8.25 \mathrm{~Hz}, 1 \mathrm{H})$, $7.43(\mathrm{~s}, 1 \mathrm{H}), 7.36(\mathrm{~s}, 1 \mathrm{H}), 7.17(\mathrm{dd}, \mathrm{J}=2.3,3.1,3.3 \mathrm{~Hz}, 1 \mathrm{H}), 7.13(\mathrm{dd}, \mathrm{J}=2.45,3.25,3.15 \mathrm{~Hz}$, 1H), $7.08(\mathrm{~s}, 1 \mathrm{H}), 6.67(\mathrm{~d}, \mathrm{~J}=16.05 \mathrm{~Hz}, 1 \mathrm{H}), 6.53(\mathrm{~d}, \mathrm{~J}=16.05 \mathrm{~Hz}, 1 \mathrm{H}), 6.51(\mathrm{~m}, 1 \mathrm{H}), 6.45(\mathrm{~m}$, $1 \mathrm{H}), 5.25(\mathrm{~d}, \mathrm{~J}=9.0 \mathrm{~Hz}, 1 \mathrm{H}), 4.22(\mathrm{~m}, 1 \mathrm{H}), 2.26(\mathrm{dd}, \mathrm{J}=6.95,12.25 \mathrm{~Hz}, 1 \mathrm{H}), 2.00(\mathrm{dd}, \mathrm{J}=10.3$, $12.35 \mathrm{~Hz}, 1 \mathrm{H}), 1.85(\mathrm{~d}, \mathrm{~J}=1.35 \mathrm{~Hz}, 3 \mathrm{H}), 1.83(\mathrm{~d}, \mathrm{~J}=1.4 \mathrm{~Hz}, 3 \mathrm{H}), 1.49(\mathrm{~s}, 3 \mathrm{H})$.

${ }^{13} \mathrm{C} \mathrm{NMR}\left(800 \mathrm{MHz}, \mathrm{CDCl}_{3}\right) \delta$ (ppm) = 143.27, 140,94, 137.81, 136.23, 135.61, 132.53, 132.28, $128.24,127.38,127.24,127.10,124.39,123.86,120.62,118.56,114.95,108.72,106.34$, 102.64, 102.43, 49.78, 48.30, 41.34, 25.95, 25.57, 18.29.

HRMS (ESI) m/z [M+H'] Calcd for $\mathrm{C}_{26} \mathrm{H}_{27} \mathrm{~N}_{2}$ 367.2174; Found 367.2166. 
DAD1 A, Sig=254,4 Ref $=360,100$ (ManoellJ
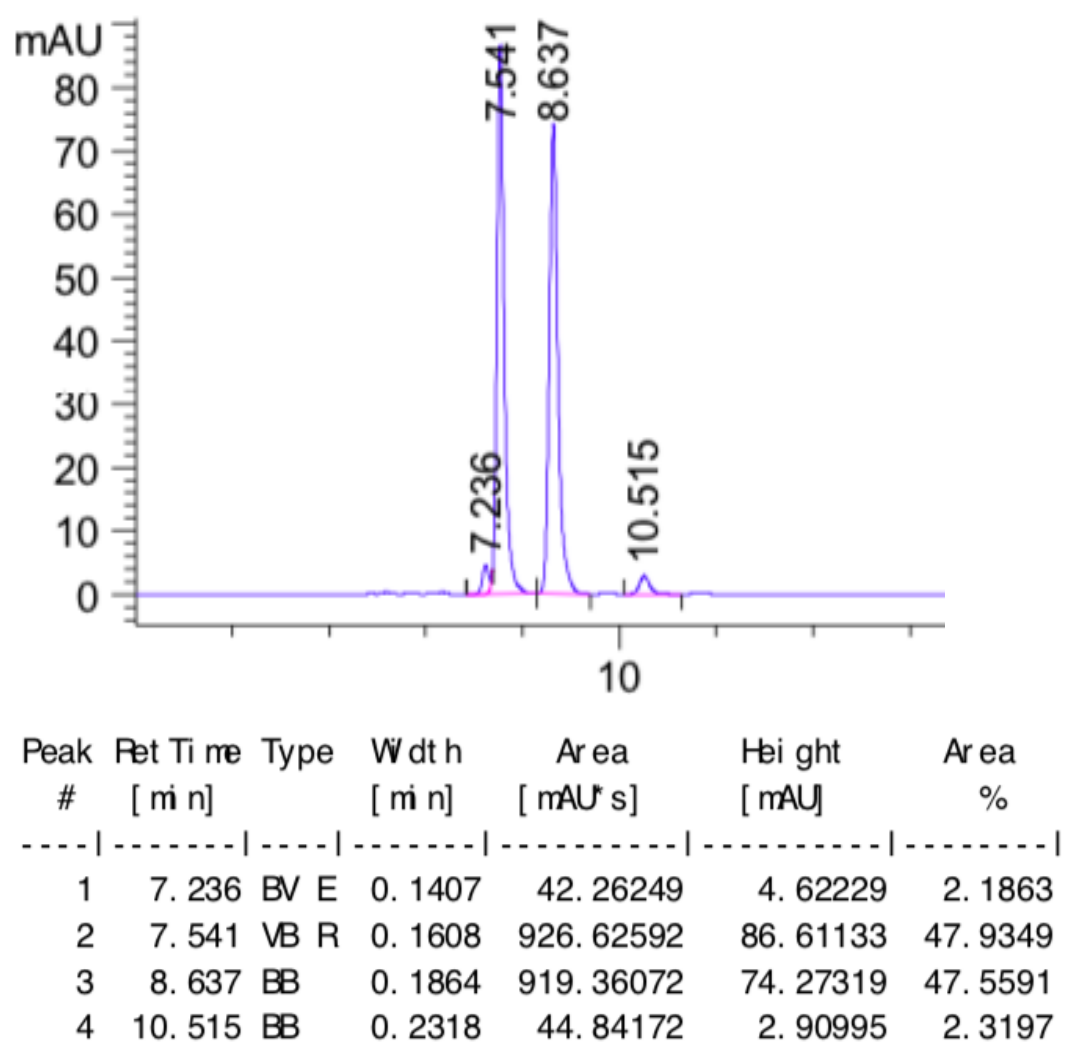

Figure 1. HPLC chromatogram of racemic acetate (+/-)-13. Chiral column Chiralpak ${ }^{\circledR}$ IA (4.6x $250 \mathrm{~mm}, 5 \mu \mathrm{m}, 10: 90 \mathrm{v} / \mathrm{v}, i-\mathrm{PrOH} /$ hexane, isocratic mode, flow $1.0 \mathrm{~mL} / \mathrm{min}$, UV detection at $254 \mathrm{~nm})$.

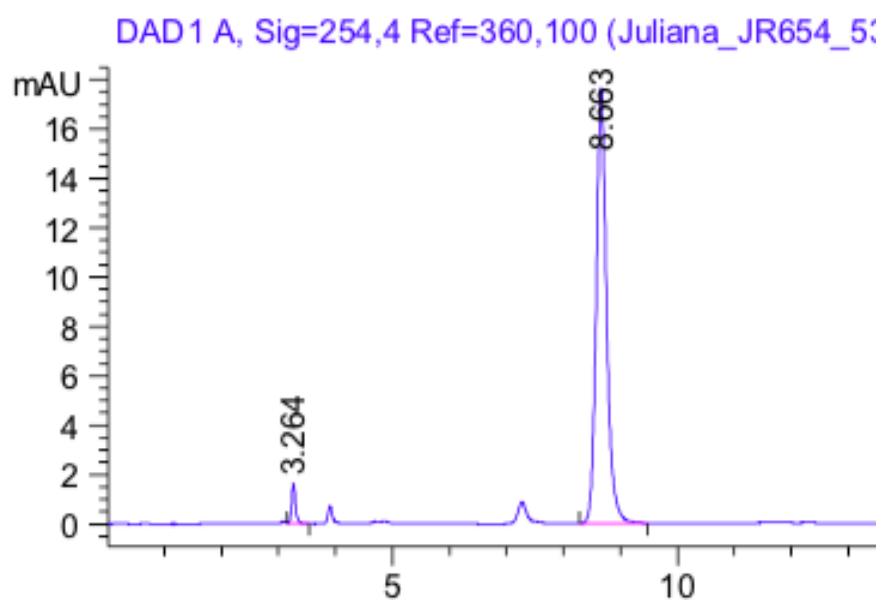




\begin{tabular}{|c|c|c|c|c|c|}
\hline $\begin{array}{c}\text { Peak } \\
\text { \# }\end{array}$ & $\begin{array}{l}\text { Ret Ti me Type } \\
{[\mathrm{m} n]}\end{array}$ & $\begin{array}{l}\text { W dth } \\
{[\mathrm{m} \mathrm{n}]}\end{array}$ & $\begin{array}{c}\text { Area } \\
{\left[\mathrm{mAU}^{*} \mathrm{~s}\right]}\end{array}$ & $\begin{array}{l}\text { Hei ght } \\
\text { [ } \mathrm{mAU}]\end{array}$ & $\begin{array}{c}\text { Ar ea } \\
\%\end{array}$ \\
\hline - & 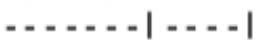 & 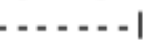 & 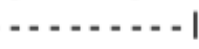 & & -1 \\
\hline 1 & 3. $264 \mathrm{BB}$ & 0.0698 & 7. 37568 & 1. 59971 & 1. 5411 \\
\hline 2 & 8. $663 \mathrm{BB}$ & 0. 1922 & 223. 98225 & 17.63116 & 46. 7990 \\
\hline 3 & 19. 222 BB & 0.4876 & 247. 24731 & 7. 63991 & 51.6600 \\
\hline
\end{tabular}

Figure 2. HPLC chromatogram of acetate (5S,7S)-13. Chiral column Chiralpak® IA (4.6 x 250 $\mathrm{mm}, 5 \mu \mathrm{m}, 10: 90 \mathrm{v} / \mathrm{v}, i-\mathrm{PrOH} /$ hexane, isocratic mode, flow $1.0 \mathrm{~mL} / \mathrm{min}$, UV detection at 254 $\mathrm{nm})$.

\section{References}

1. Perrin, D. D., Amarego, W. L. F. Purification of laboratory chemicals. 1988. 3 rd ed. Oxford, New York, Pergamon Press.

2. Nishimura, T., Yasuhara, Y., Hayashi, T. Iridium-Catalyzed [3 + 2] Annulation of 1,3-Dienes with ortho-Carbonylated Phenylboronic Acids. A Catalytic Process Involving Regioselective 1,2-Addition. J. Am. Chem. Soc. 2007. 129, 24, 7506-7507.

3. Marković, D., Tchawou, W. A., Novosjolova, I., Laclef, S., Stepanovs, D., Turks, M., Vogel, P. Synthesis and Applications of Silyl 2-Methylprop-2-ene-1-sulfinates in Preparative Silylation and GC-Derivatization Reactions of Polyols and Carbohydrates. Chem. Eur. J. 2016. 22, 12, 4196-4205.

4. Anbu, N., Nagarjun, N., Jacob, M., Kalaiarasi, J. M. V., Dhakshinamoorthy, A. Acetylation of Alcohols, Amines, Phenols, Thiols under Catalyst and Solvent-Free Conditions. Chemistry. 2019. 1, 1, 69-79.

5. Kock, M., Jones, P. G., Lindel, T. Total Synthesis and Absolute Configuration of Raputindole A. Org. Lett. 2017. 19, 23, 6296-6299.

14. Kock, M., Lindel, T., Diastereoselective Total Synthesis of Raputindole A. Org. Lett. 2018. $20,17,5444-5447$. 
NMR Spectra

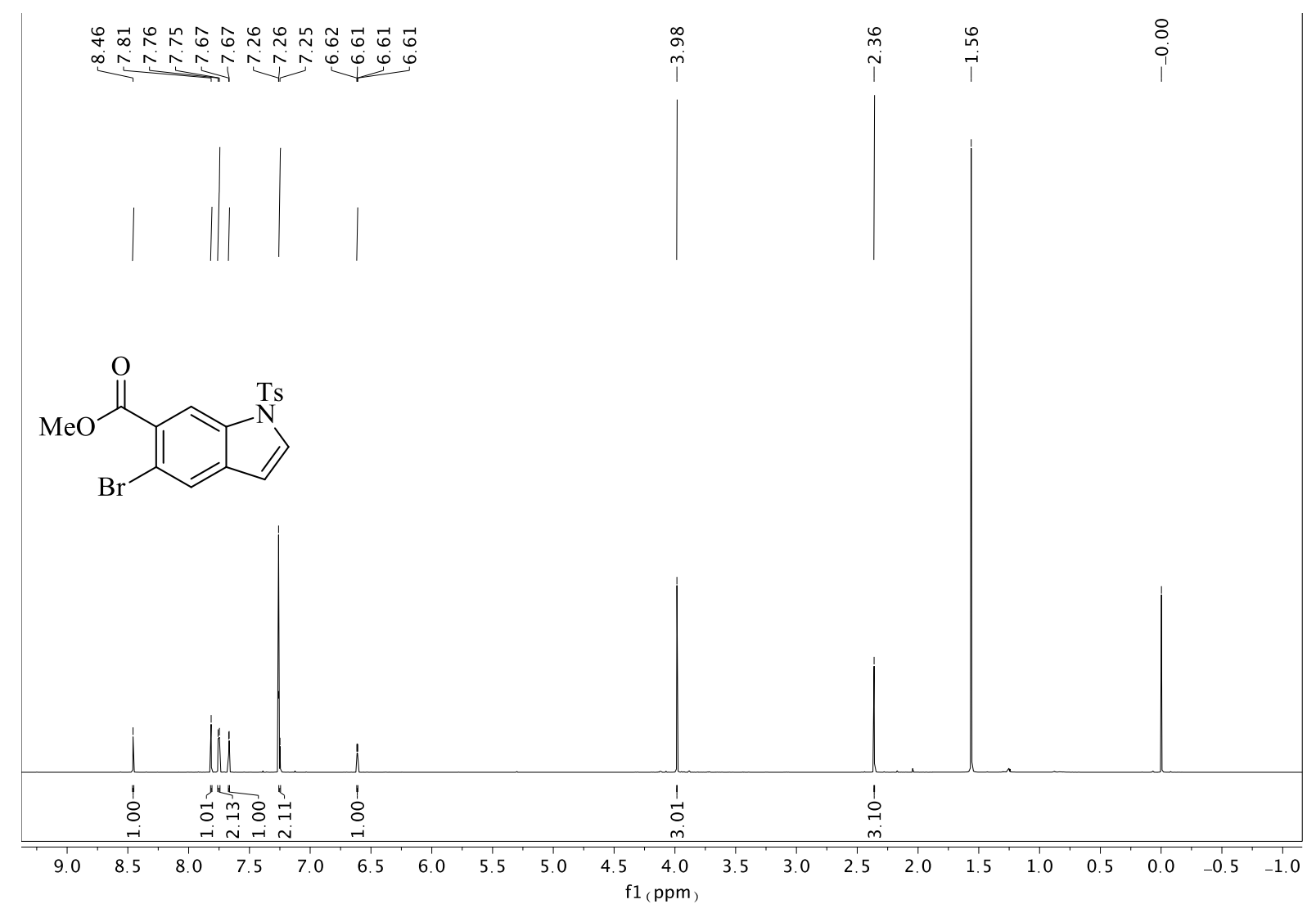

${ }^{1} \mathrm{H} \mathrm{NMR}\left(800 \mathrm{MHz}, \mathrm{CDCl}_{3}\right.$ ) Spectrum of Compound 8b. (residual water: in $1.55 \mathrm{ppm}$ ). 


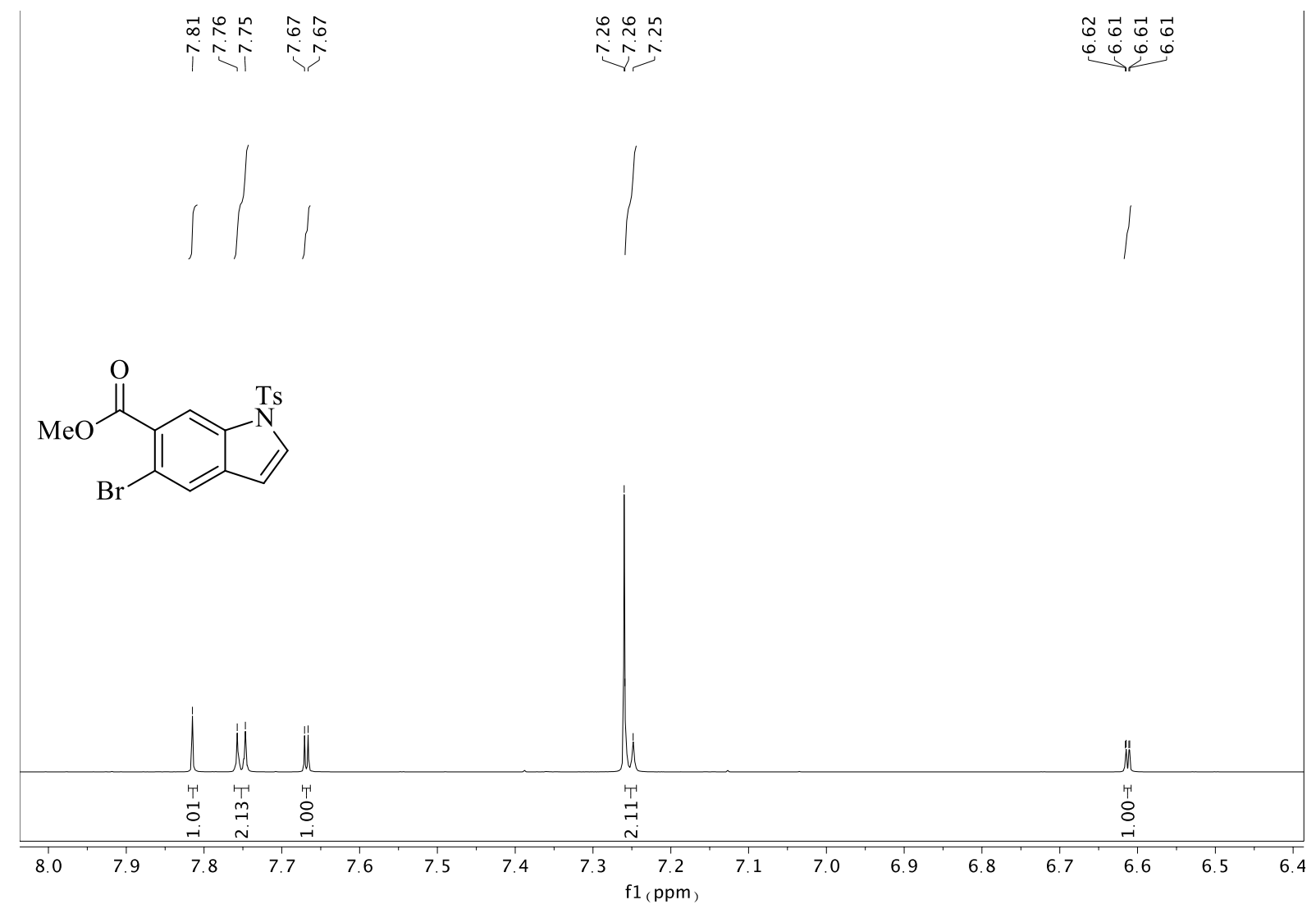

${ }^{1} \mathrm{H}$ NMR (800 MHz, $\mathrm{CDCl}_{3}$ ) Spectrum of Compound $\mathbf{8 b}$.

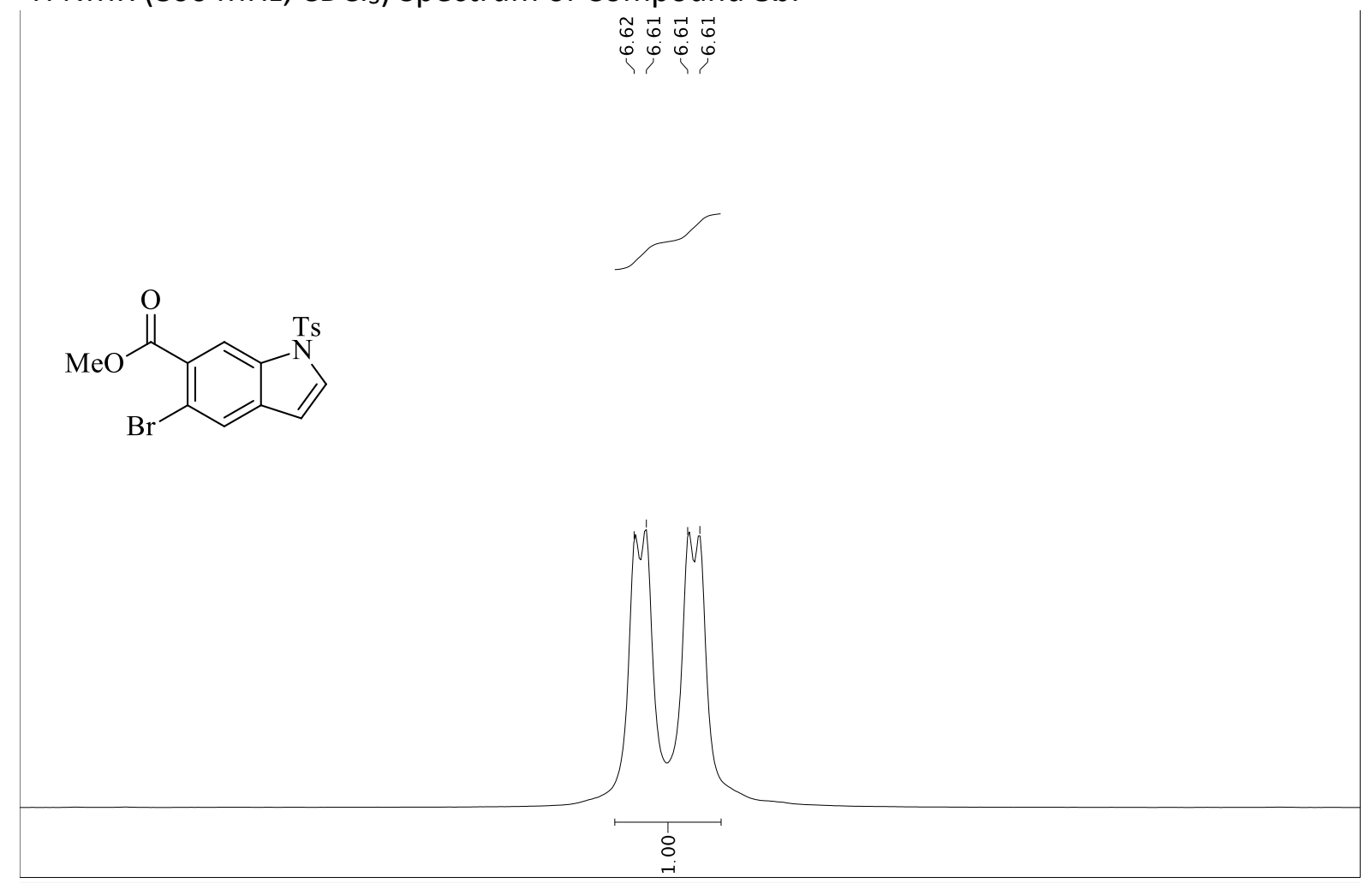

6.6656 .6606 .6556 .6506 .6456 .6406 .6356 .6306 .6256 .6206 .6156 .6106 .6056 .6006 .5956 .5906 .5856 .5806 .5756 .5706 .5656 .5606 .55 $\mathrm{f} 1(\mathrm{ppm})$

${ }^{1} \mathrm{H} \mathrm{NMR}\left(800 \mathrm{MHz}, \mathrm{CDCl}_{3}\right)$ Spectrum of Compound $\mathbf{8 b}$. 


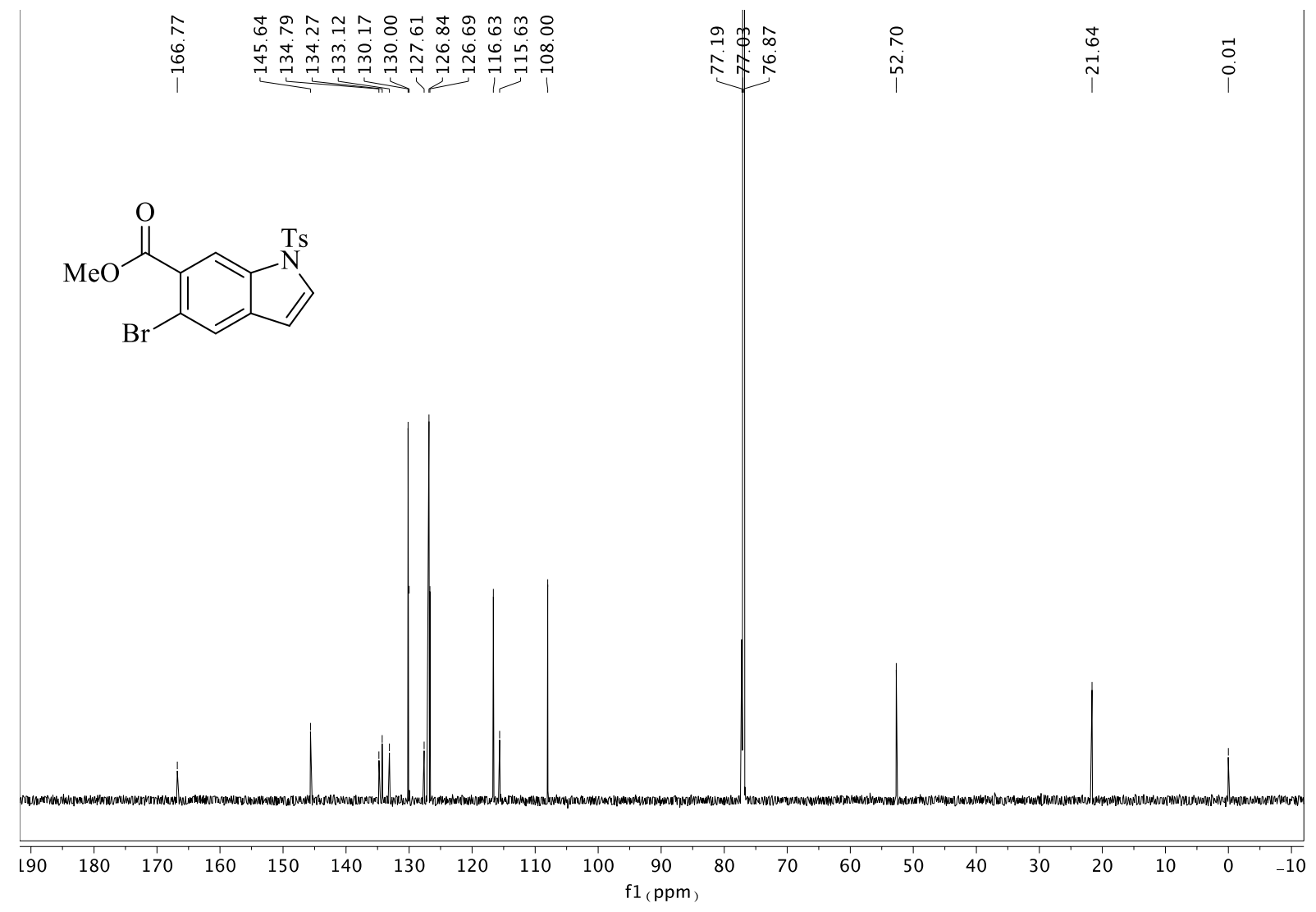

${ }^{13} \mathrm{C} \mathrm{NMR} \mathrm{(200} \mathrm{MHz,} \mathrm{CDCl}_{3}$ ) Spectrum of Compound $\mathbf{8 b}$.

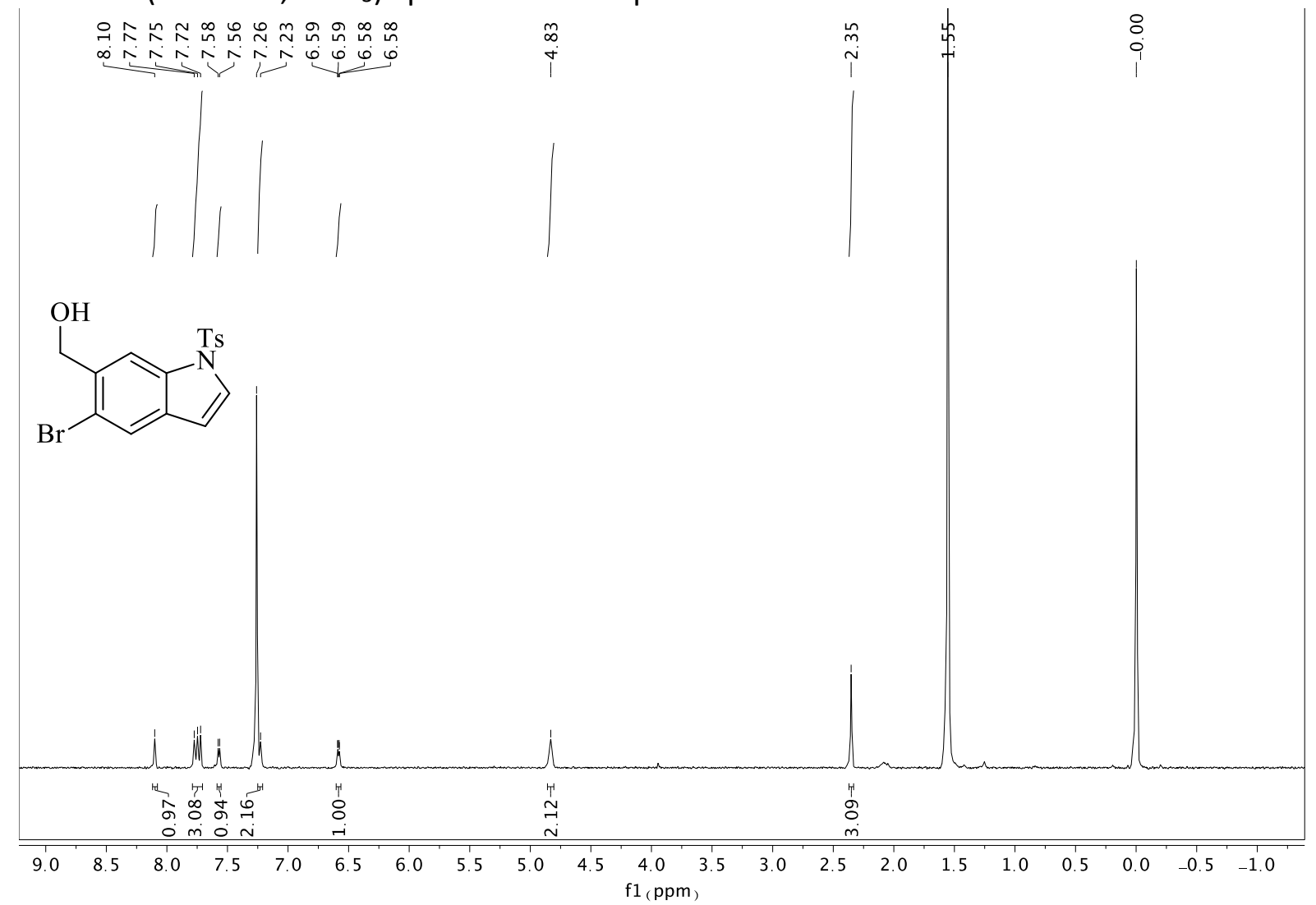

${ }^{1} \mathrm{H}$ NMR (300 MHz, $\mathrm{CDCl}_{3}$ ) Spectrum of Compound 10. (residual water: $1.55 \mathrm{ppm}$ ). 


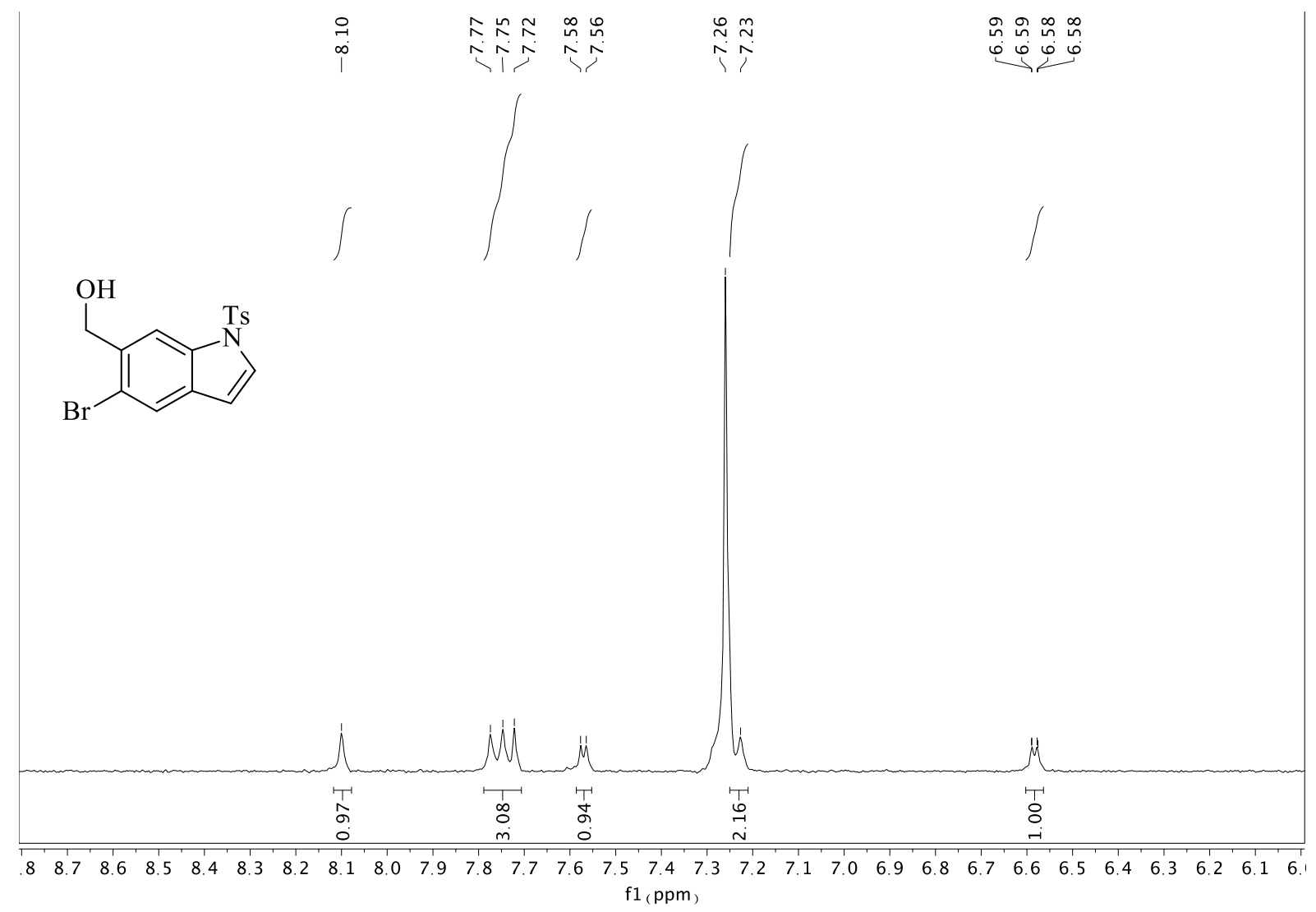

${ }^{1} \mathrm{H}$ NMR (300 MHz, $\mathrm{CDCl}_{3}$ ) Spectrum of Compound 10.

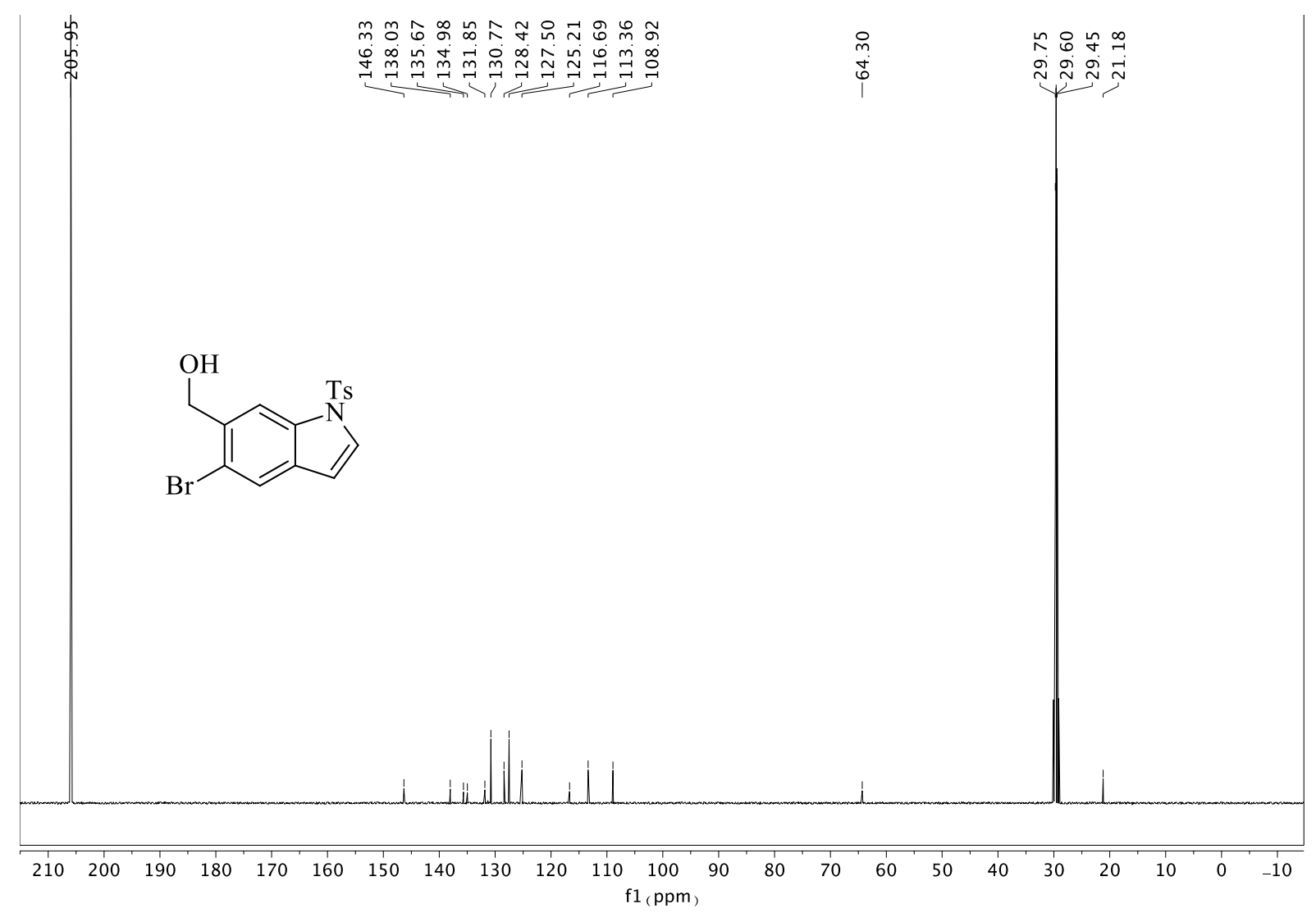

${ }^{13} \mathrm{C}$ NMR $\left(126 \mathrm{MHz}, d_{6}-\left(\mathrm{CD}_{3}\right)_{2} \mathrm{CO}\right)$ Spectrum of Compound 10. 


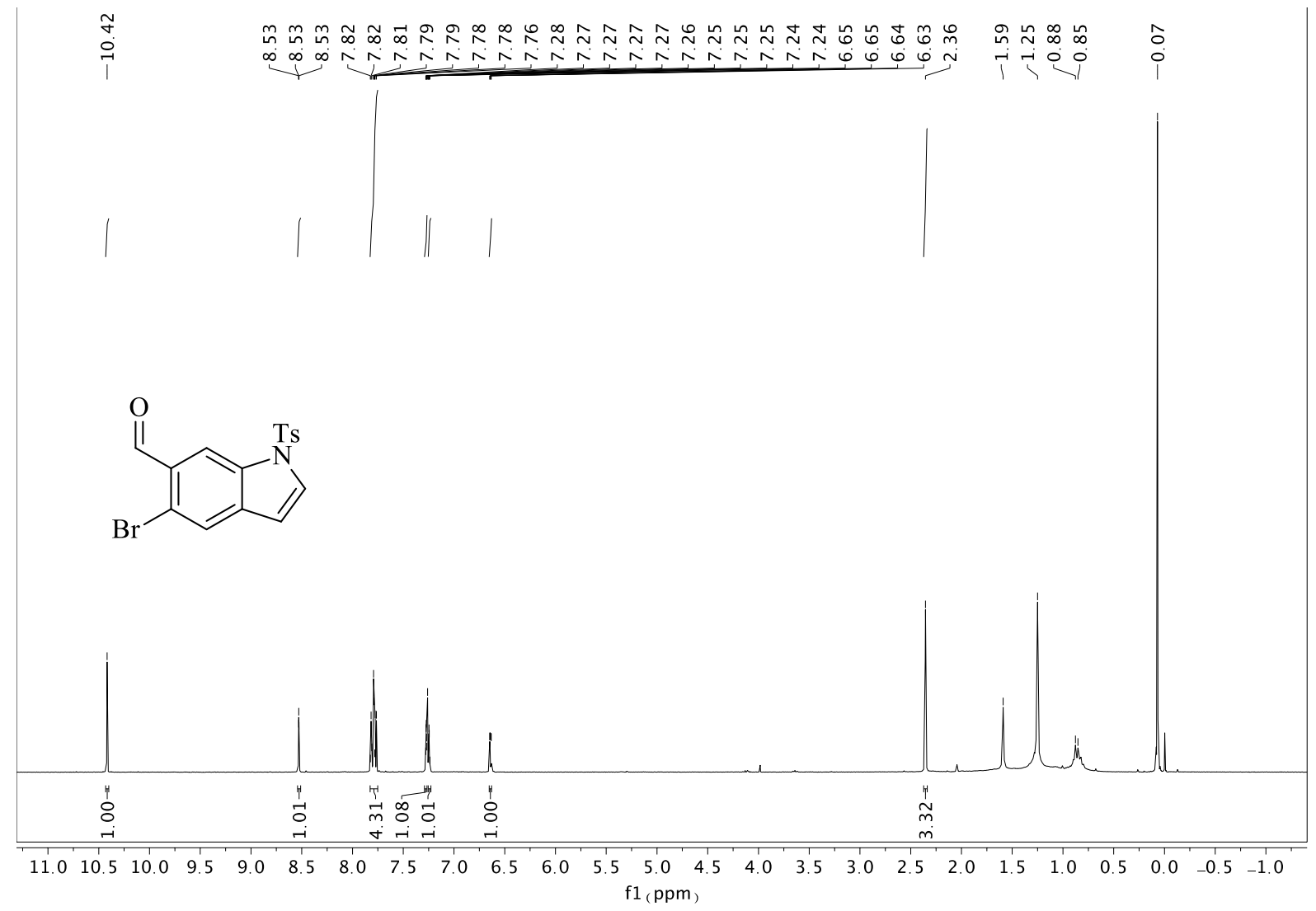

${ }^{1} \mathrm{H}$ NMR $\left(300 \mathrm{MHz}, \mathrm{CDCl}_{3}\right.$ ) Spectrum of Compound 11. (residual water: $1.59 \mathrm{ppm}$, residual hexanes grease: 1.25 and $0.88 \mathrm{ppm}$; residual silicon grease: $0.07 \mathrm{ppm}$ ).

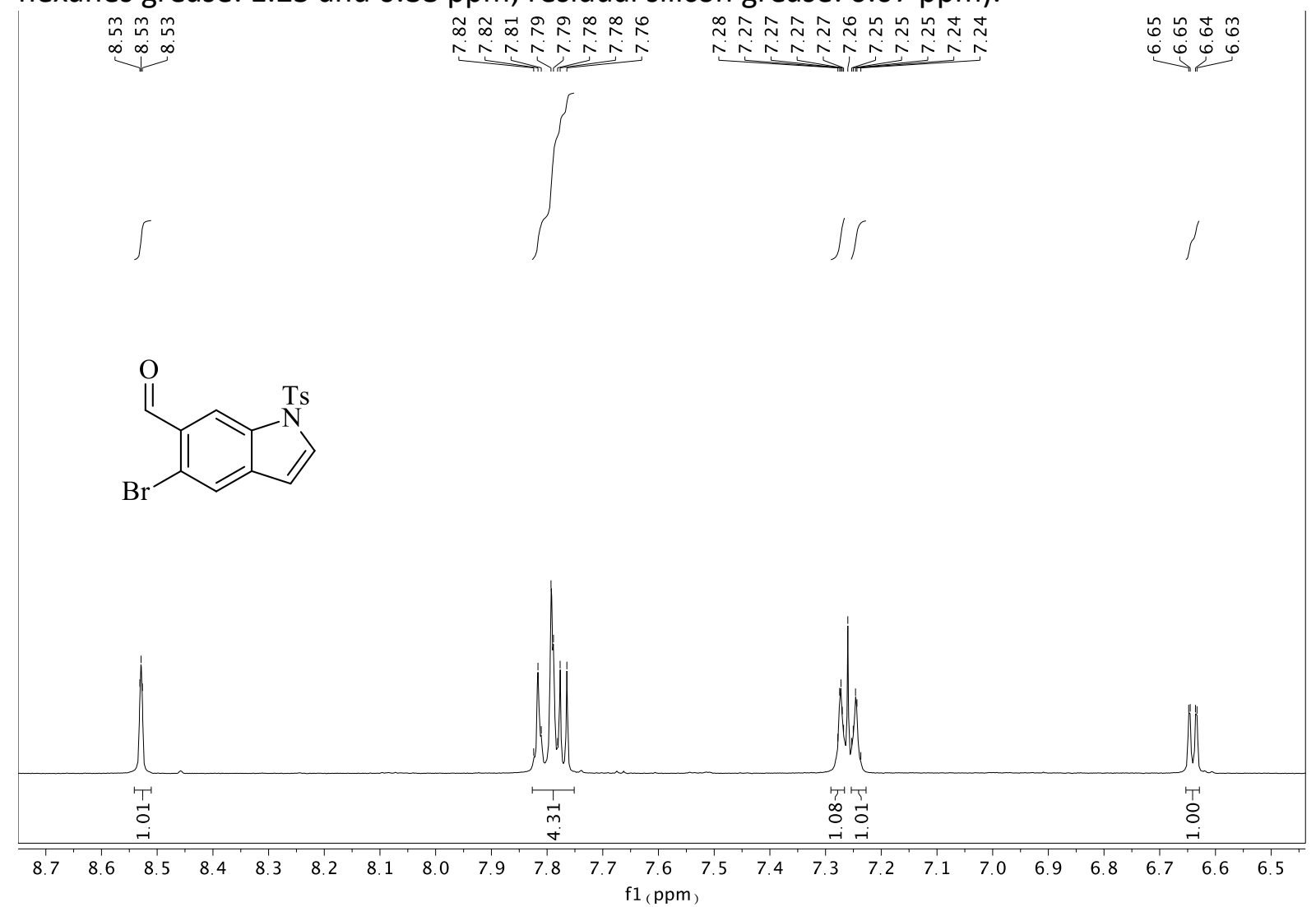

${ }^{1} \mathrm{H} \mathrm{NMR}\left(300 \mathrm{MHz}, \mathrm{CDCl}_{3}\right.$ ) Spectrum of Compound 11. 


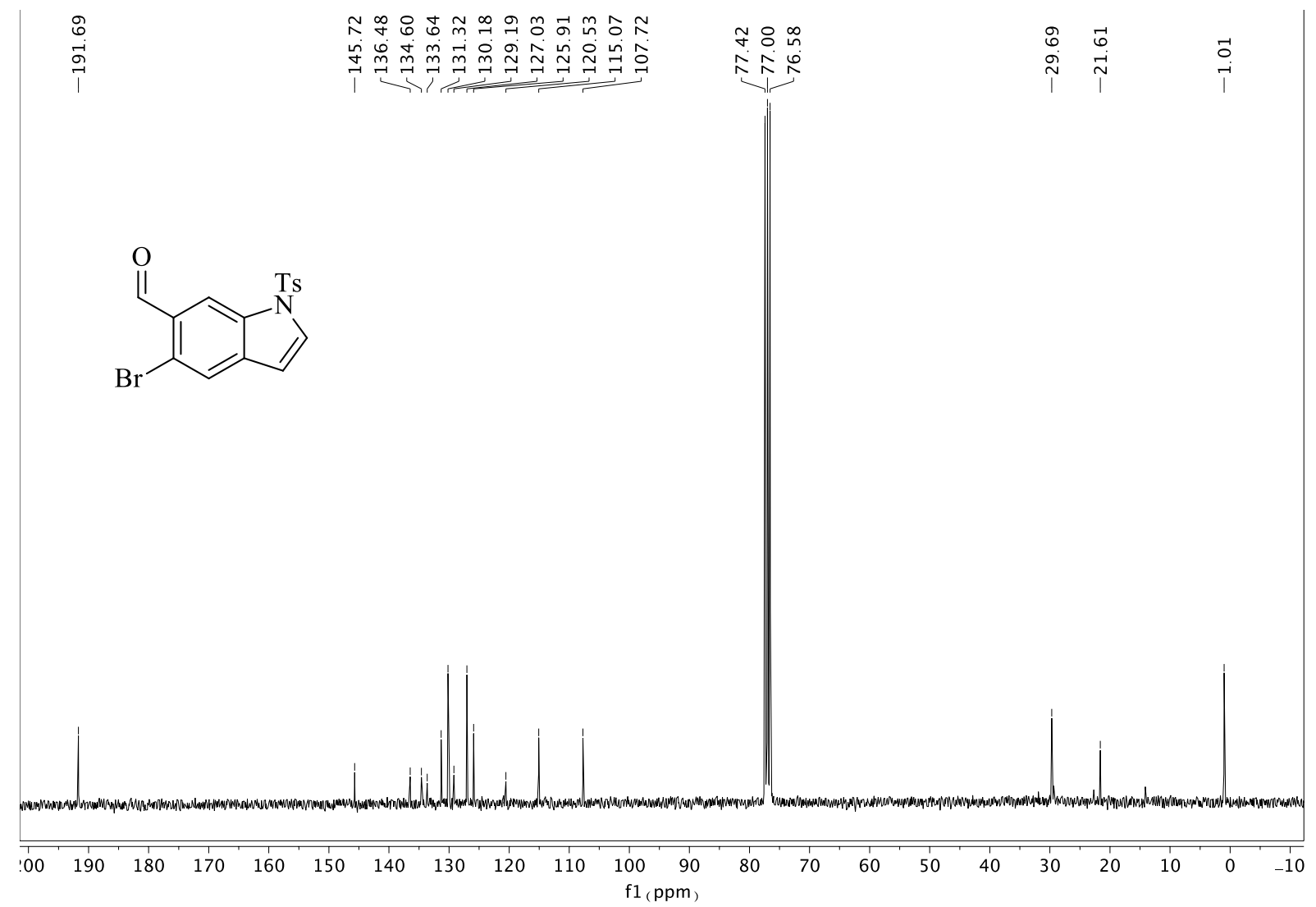

${ }^{13} \mathrm{C}$ NMR (75 MHz, $\mathrm{CDCl}_{3}$ ) Spectrum of Compound 11. (residual hexanes grease: $29.7 \mathrm{ppm}$ ).

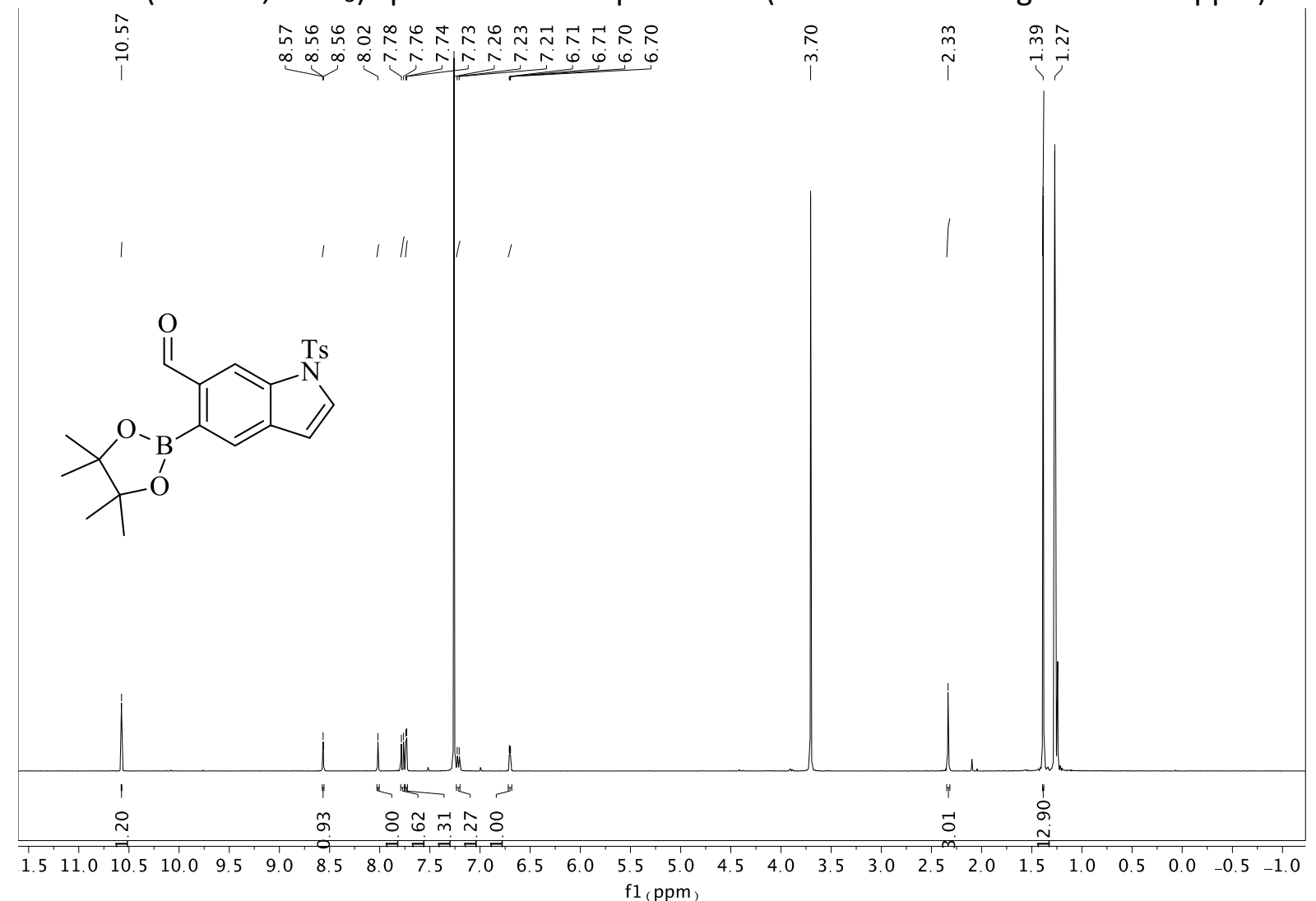

${ }^{1} \mathrm{H}$ NMR (400 MHz, $\mathrm{CDCl}_{3}$ ) Spectrum of Compound 12. (dioxane: $3.70 \mathrm{ppm}$; residual hexanes grease: $1.27 \mathrm{ppm})$. 


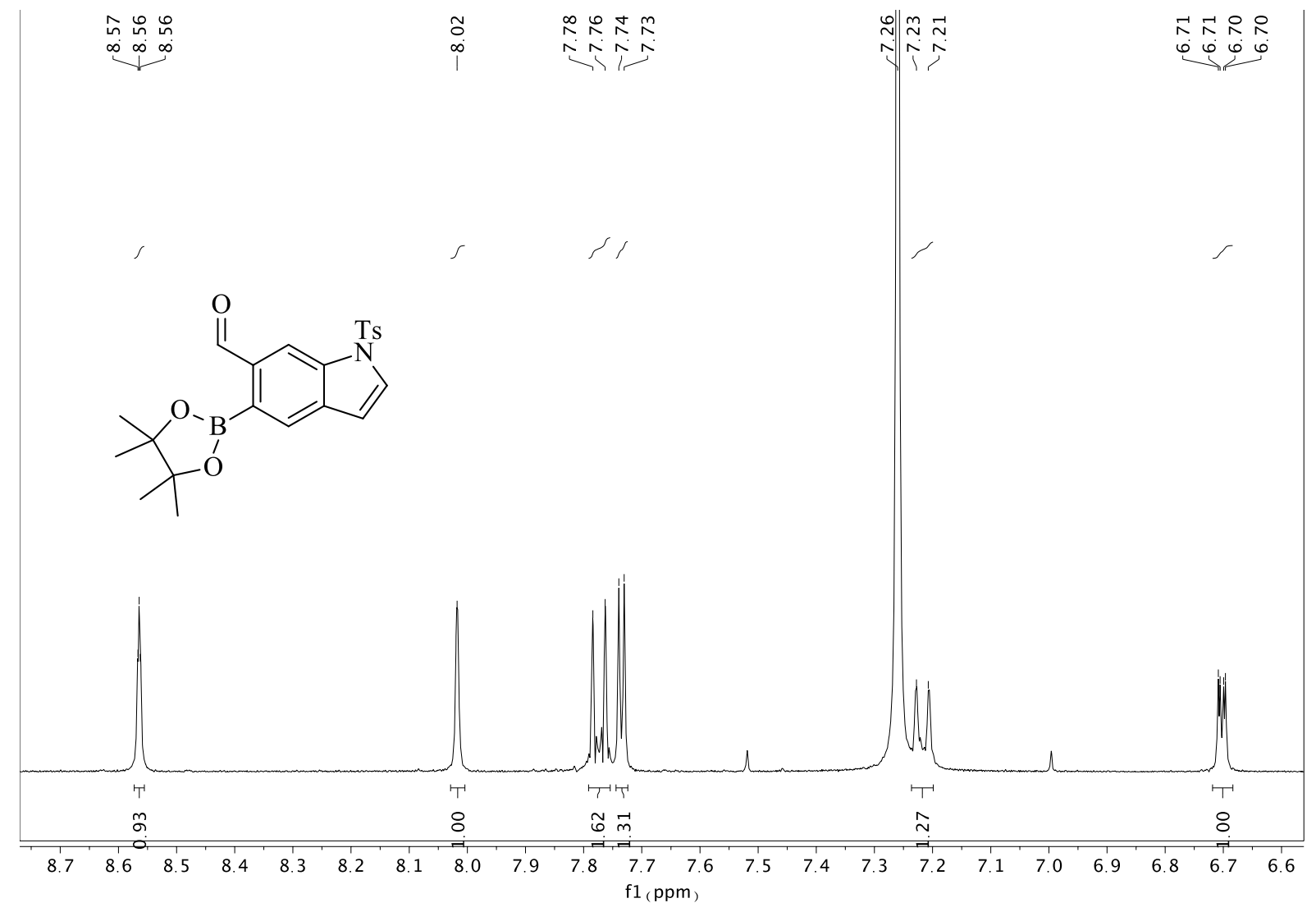

${ }^{1} \mathrm{H}$ NMR (400 MHz, $\mathrm{CDCl}_{3}$ ) Spectrum of Compound 12.

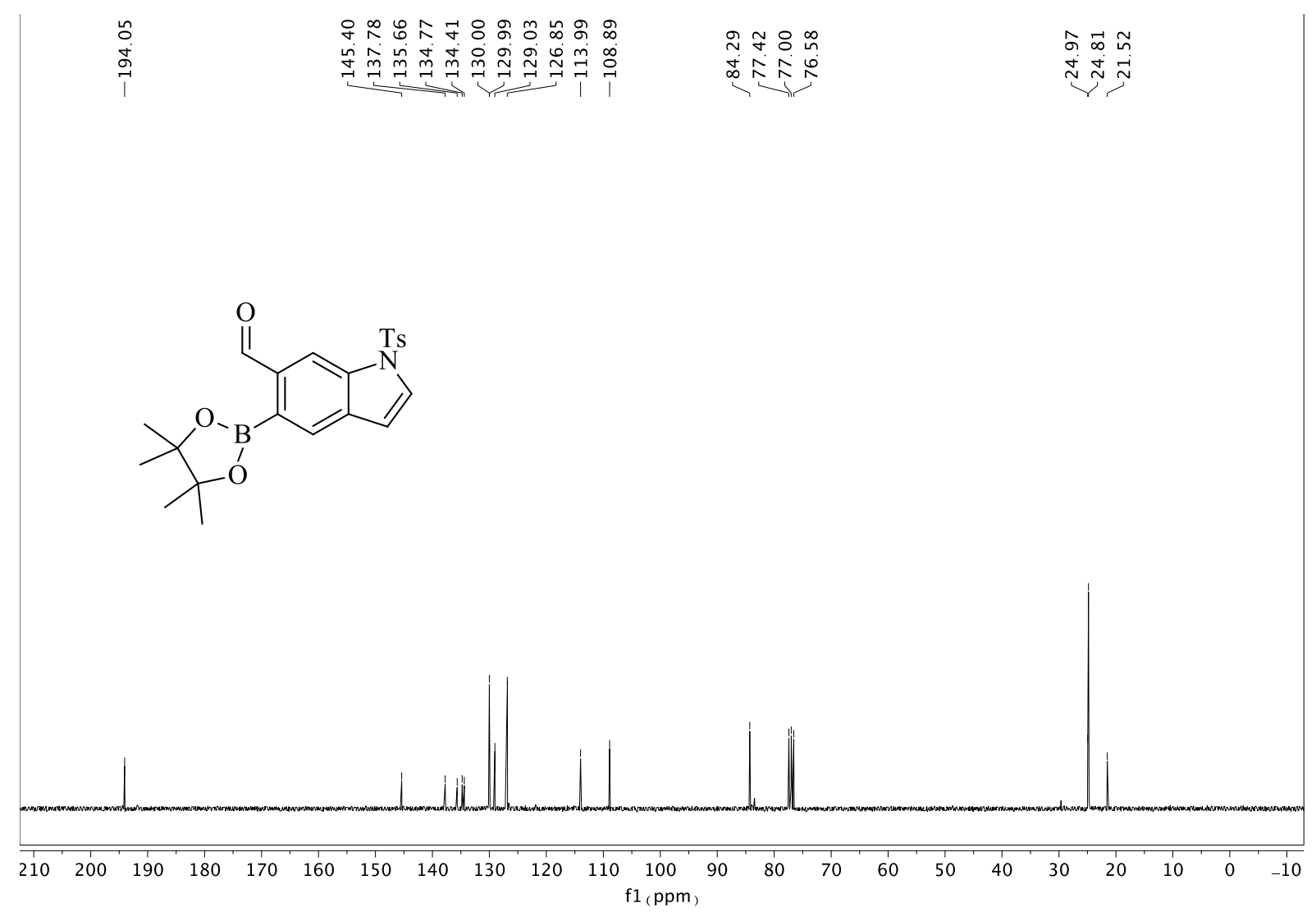

${ }^{13} \mathrm{C} \mathrm{NMR}\left(75 \mathrm{MHz}, \mathrm{CDCl}_{3}\right.$ ) Spectrum of Compound 12. 


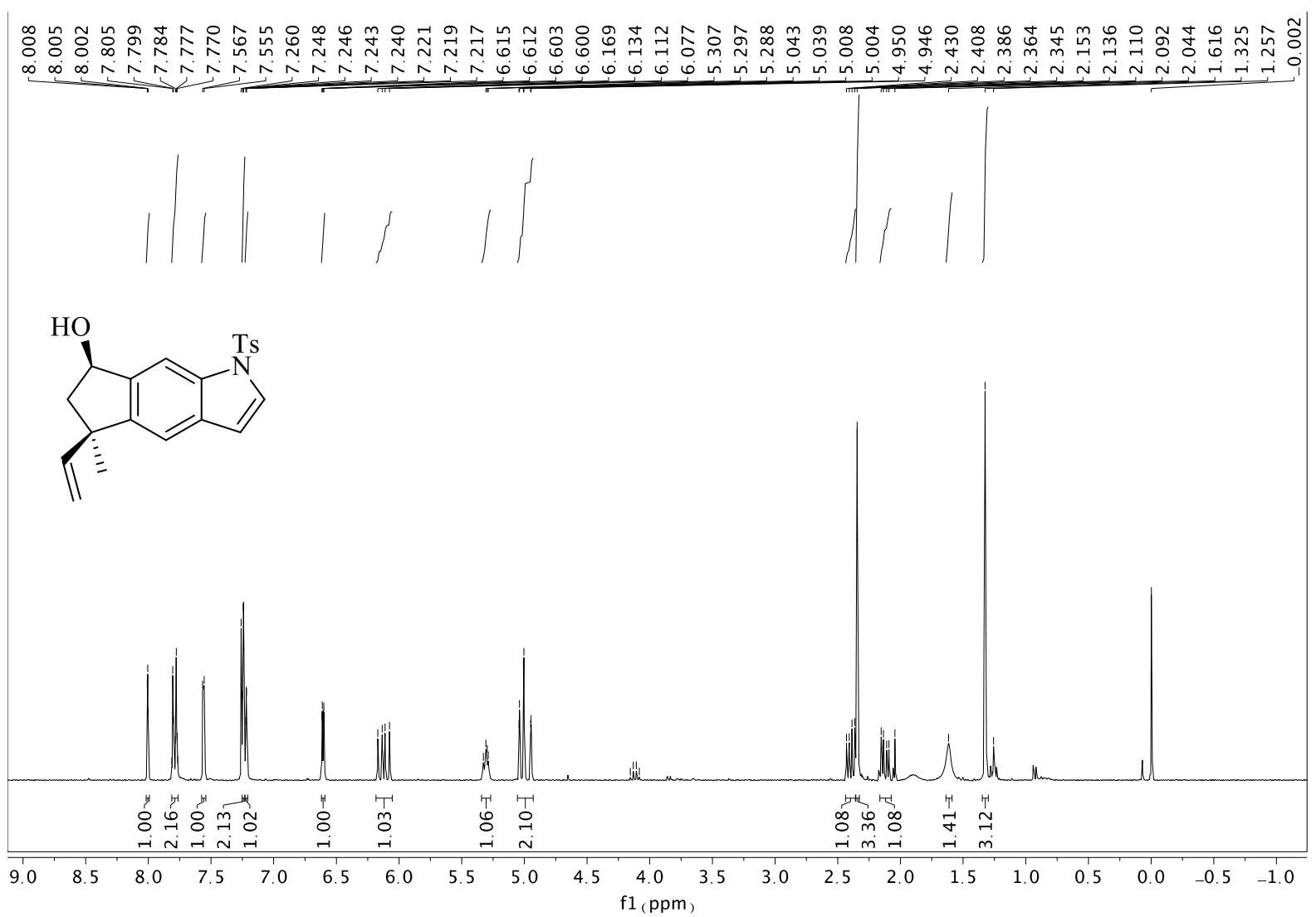

${ }^{1} \mathrm{H}$ NMR (300 MHz, $\mathrm{CDCl}_{3}$ ) Spectrum of Compound (+/-)-6. (AcOEt: 4.12, 2.5 and $1.26 \mathrm{ppm}$ ).

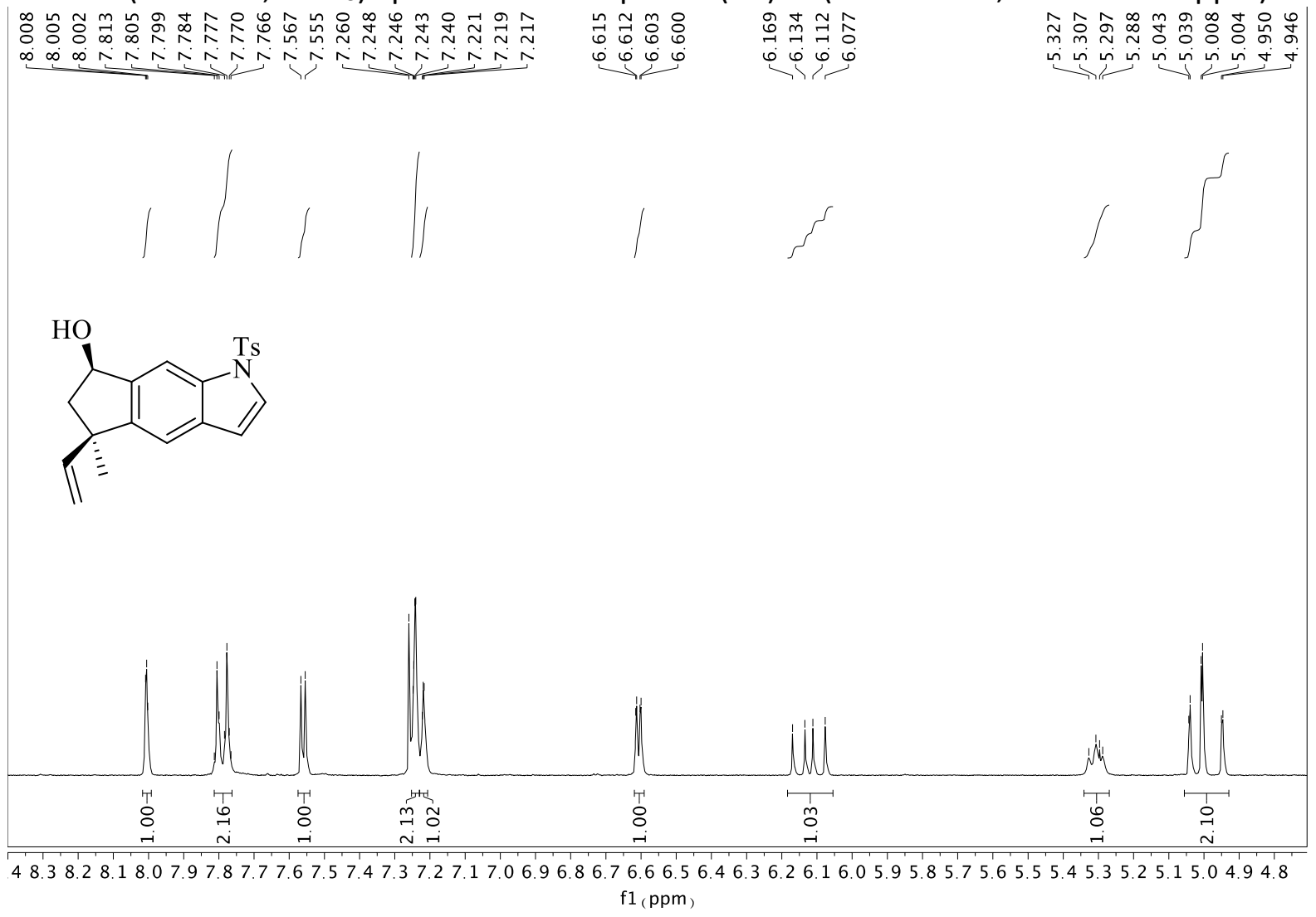

${ }^{1} \mathrm{H} \mathrm{NMR}\left(300 \mathrm{MHz}, \mathrm{CDCl}_{3}\right.$ ) Spectrum of Compound (+/-)-6. 


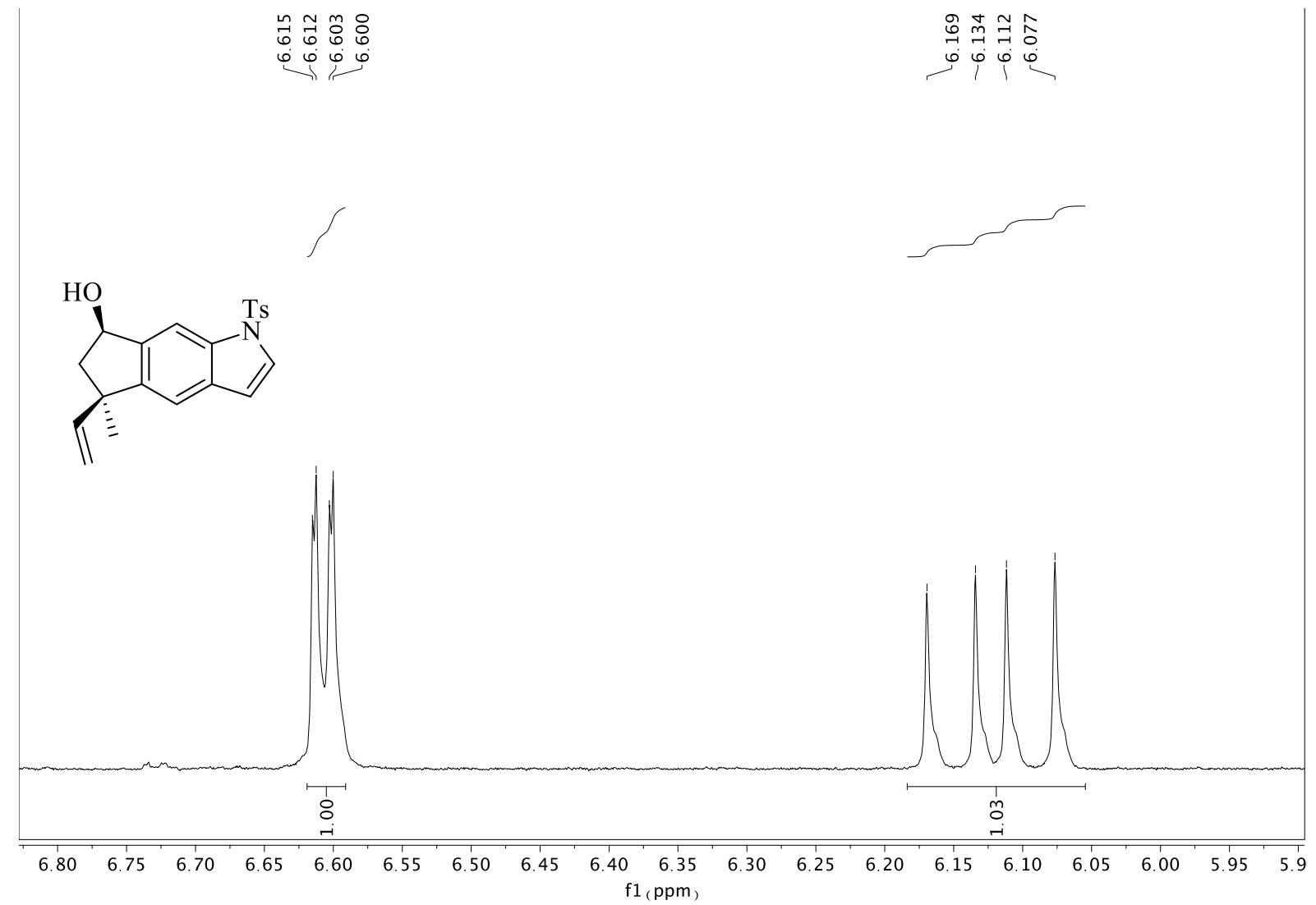

${ }^{1} \mathrm{H}$ NMR (300 MHz, $\mathrm{CDCl}_{3}$ ) Spectrum of Compound (+/-)-6.

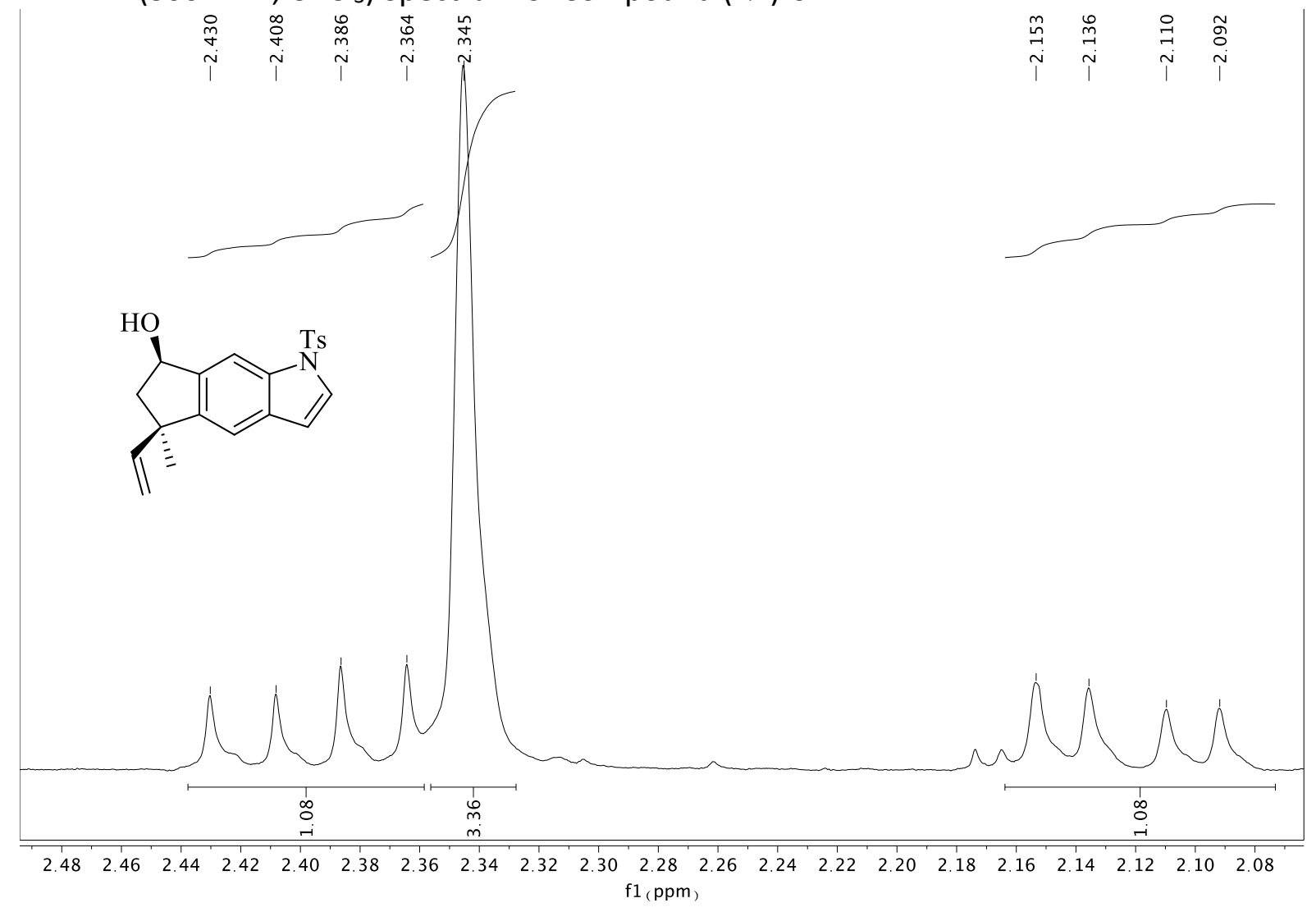

${ }^{1} \mathrm{H}$ NMR (300 MHz, $\mathrm{CDCl}_{3}$ ) Spectrum of Compound (+/-)-6. 


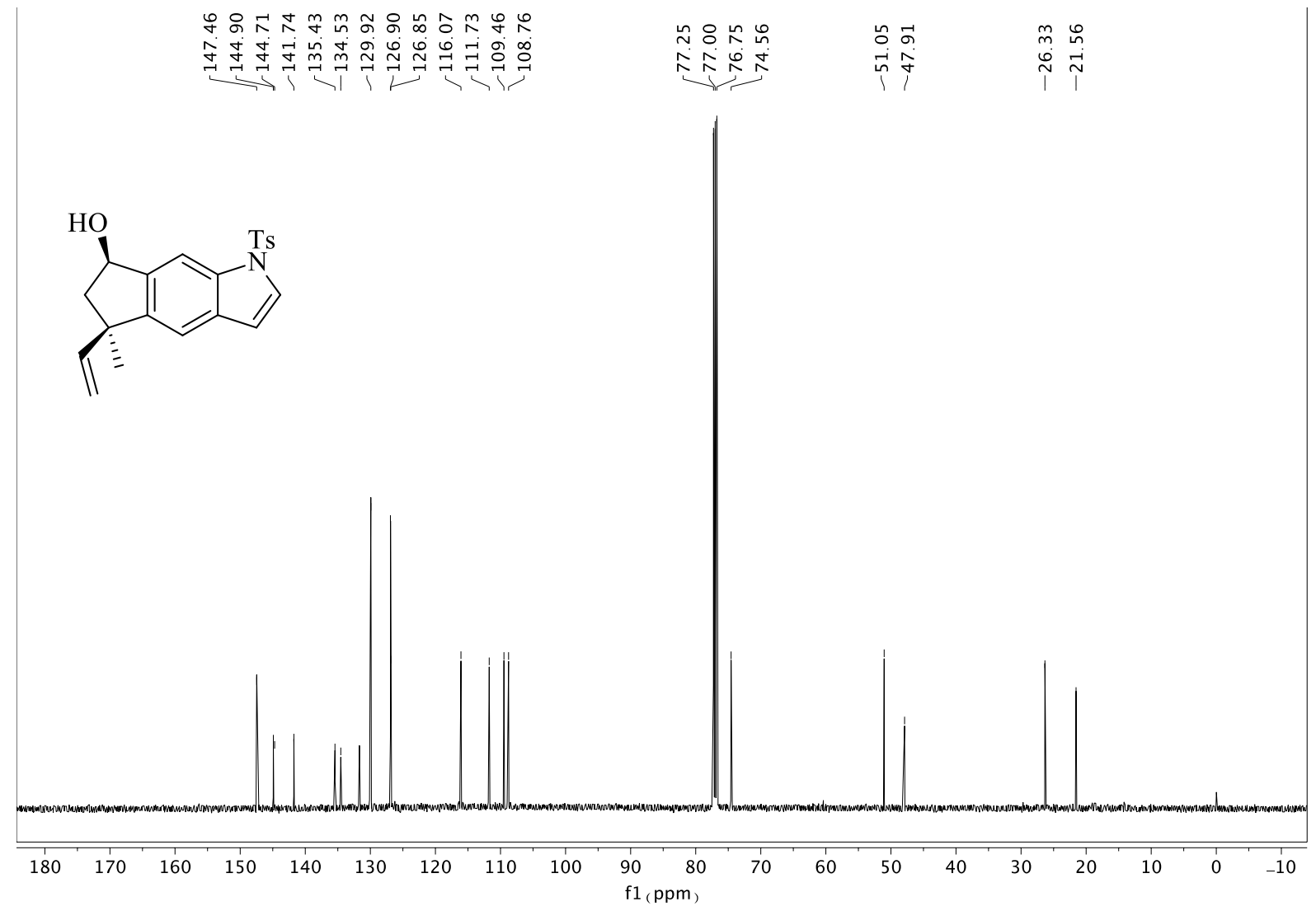

${ }^{13} \mathrm{C}$ NMR (126 MHz, $\mathrm{CDCl}_{3}$ ) Spectrum of Compound (+/-)-6.

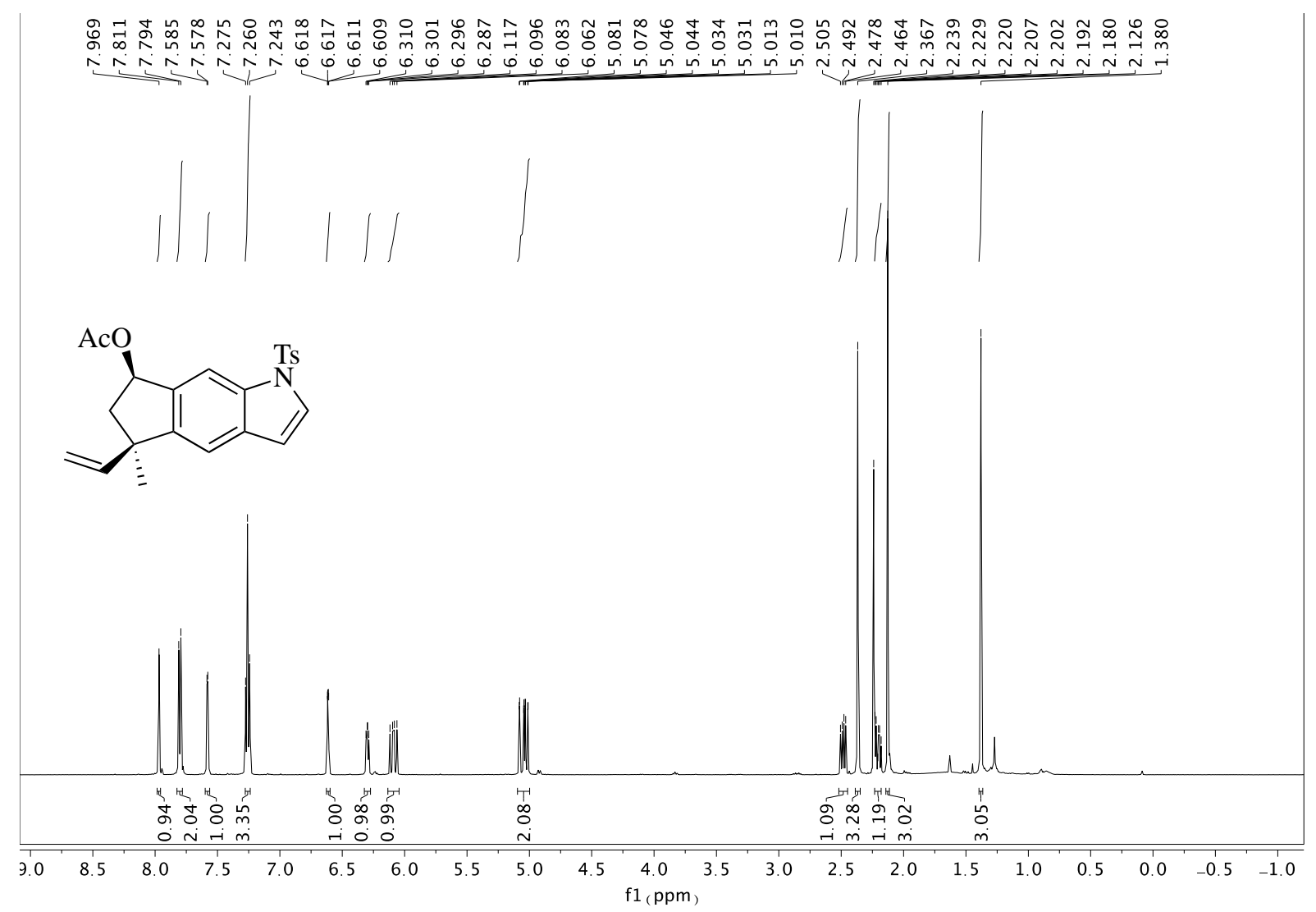

${ }^{1} \mathrm{H}$ NMR $\left(500 \mathrm{MHz}, \mathrm{CDCl}_{3}\right.$ ) Spectrum of Compound (+/-)-13. 


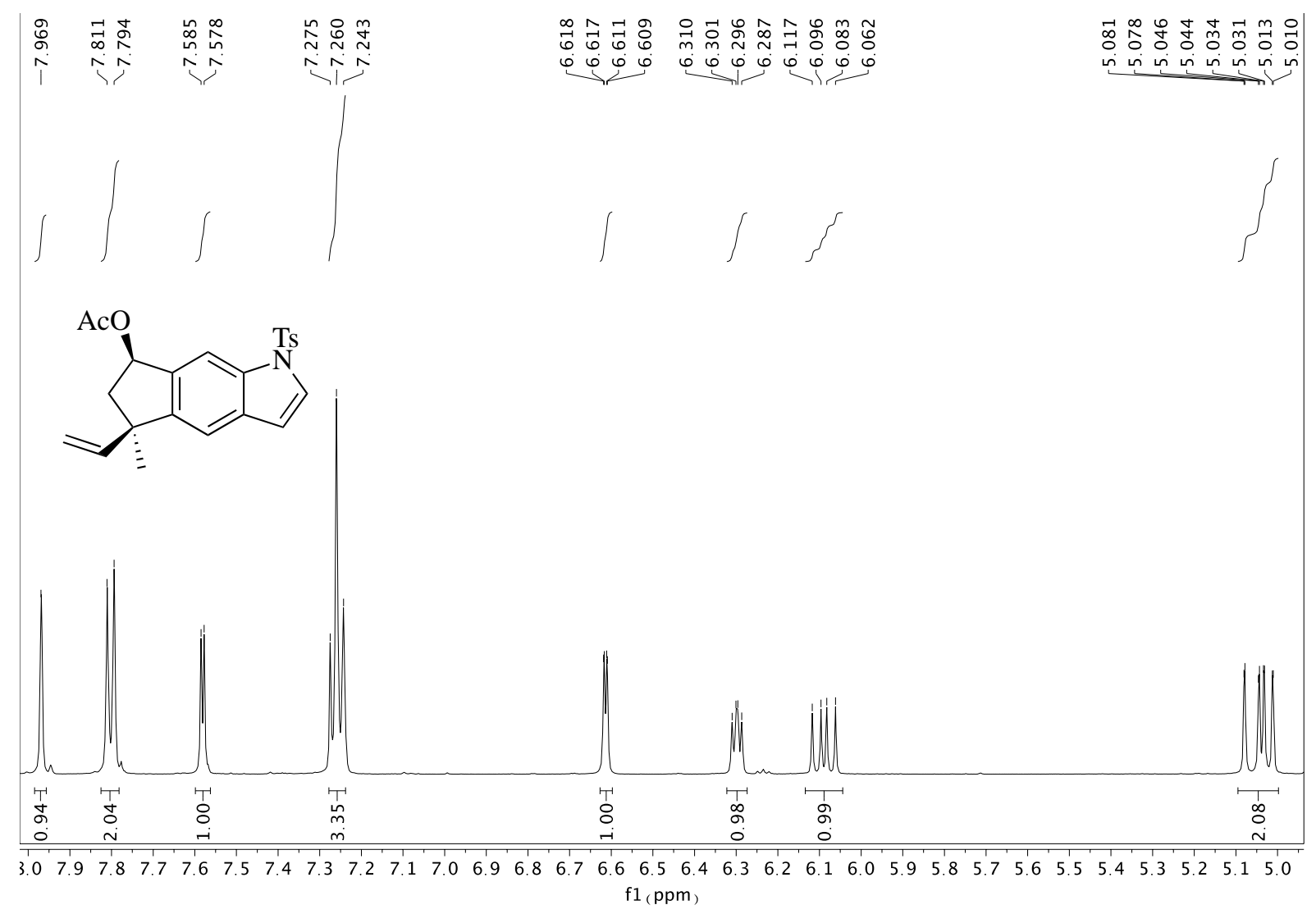

${ }^{1} \mathrm{H}$ NMR $\left(500 \mathrm{MHz}, \mathrm{CDCl}_{3}\right.$ ) Spectrum of Compound (+/-)-13.

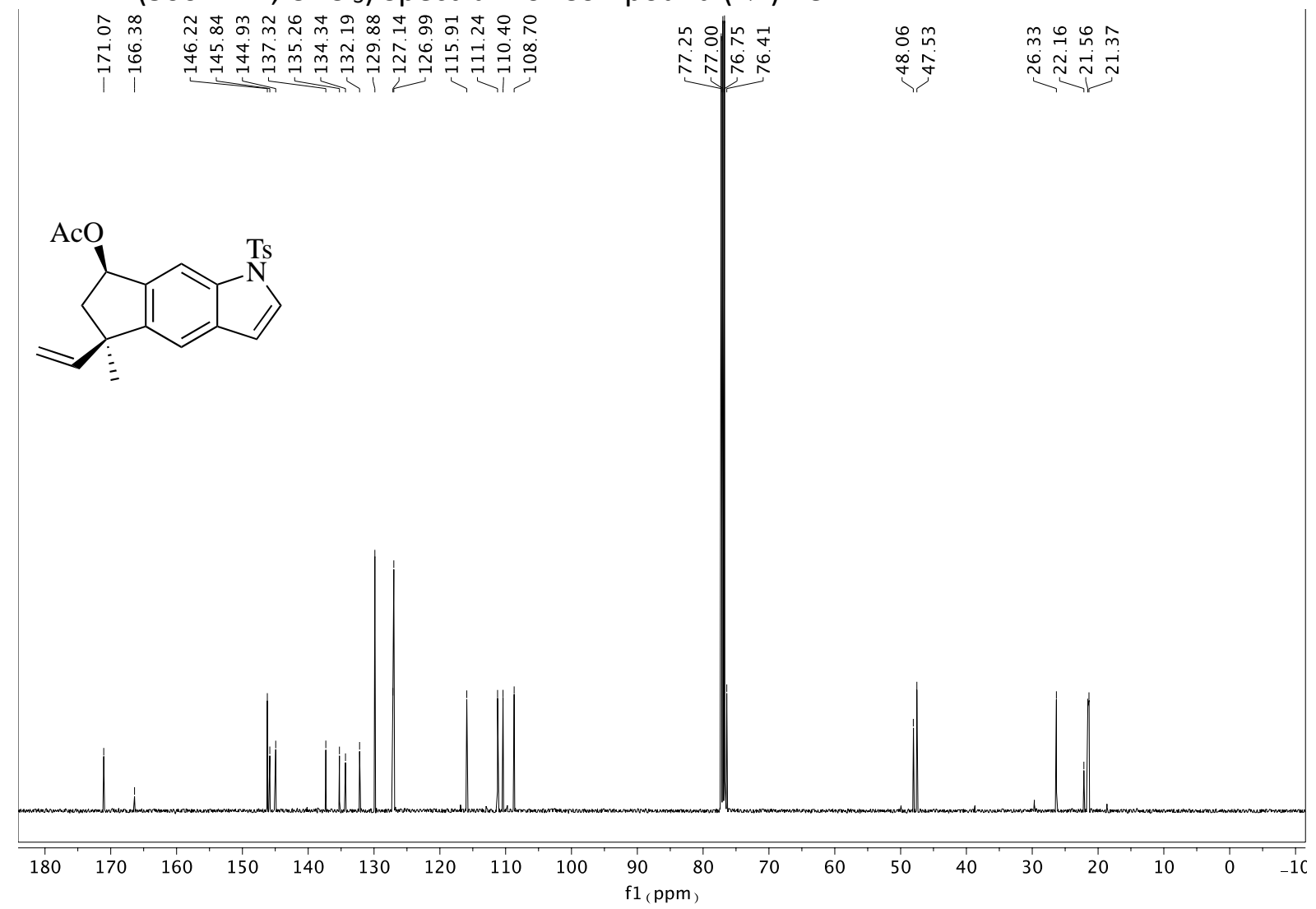

${ }^{13} \mathrm{C} \mathrm{NMR} \mathrm{(126} \mathrm{MHz,} \mathrm{CDCl}_{3}$ ) Spectrum of Compound (+/-)-13. (N,N-DMA: 171.07 and 21.37). 


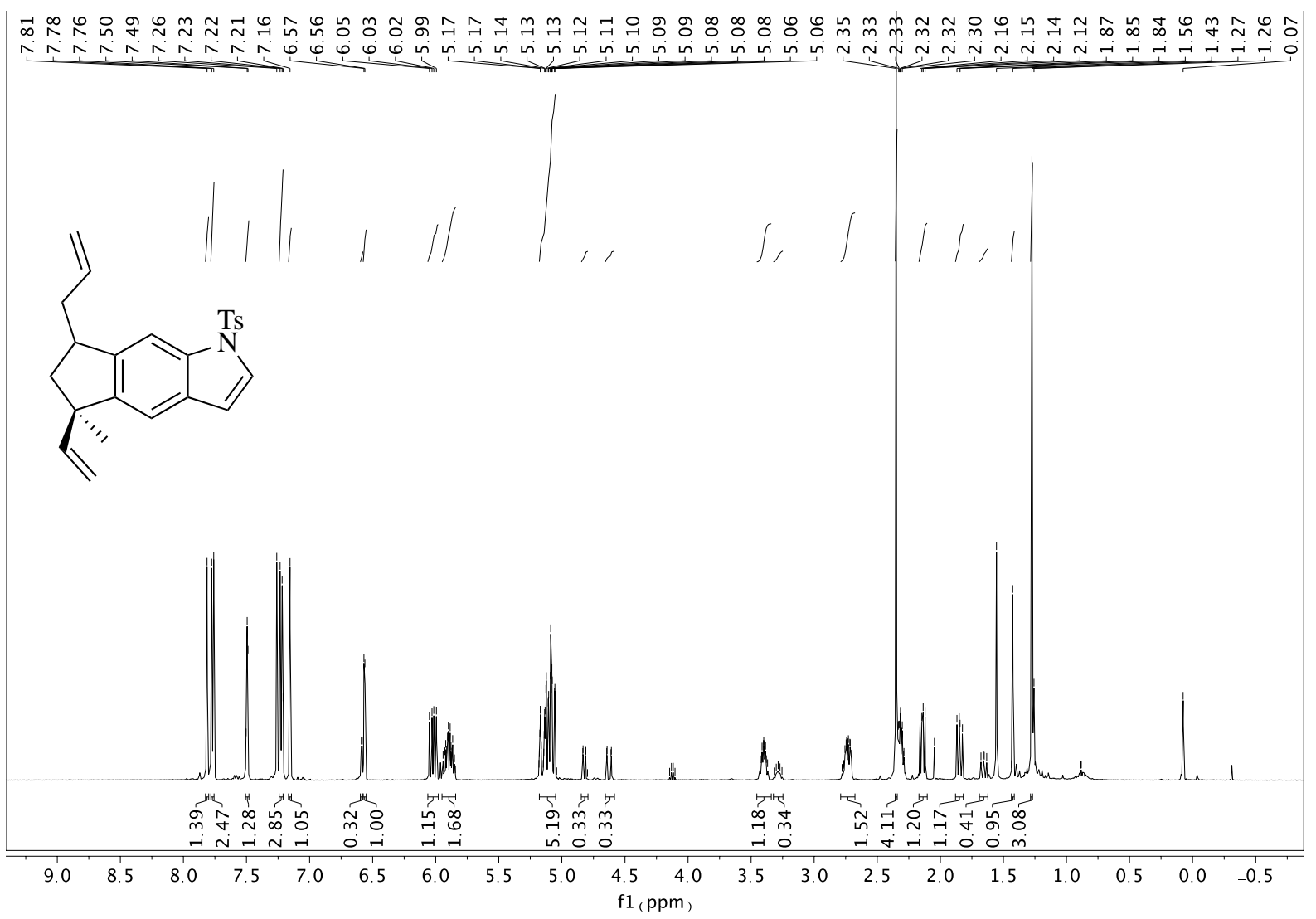

${ }^{1} \mathrm{H}$ NMR (500 MHz, $\mathrm{CDCl}_{3}$ ) Spectrum of a mixture la:Ib. (AcOEt: 4.12, 2.5 and $1.26 \mathrm{ppm}$ ).

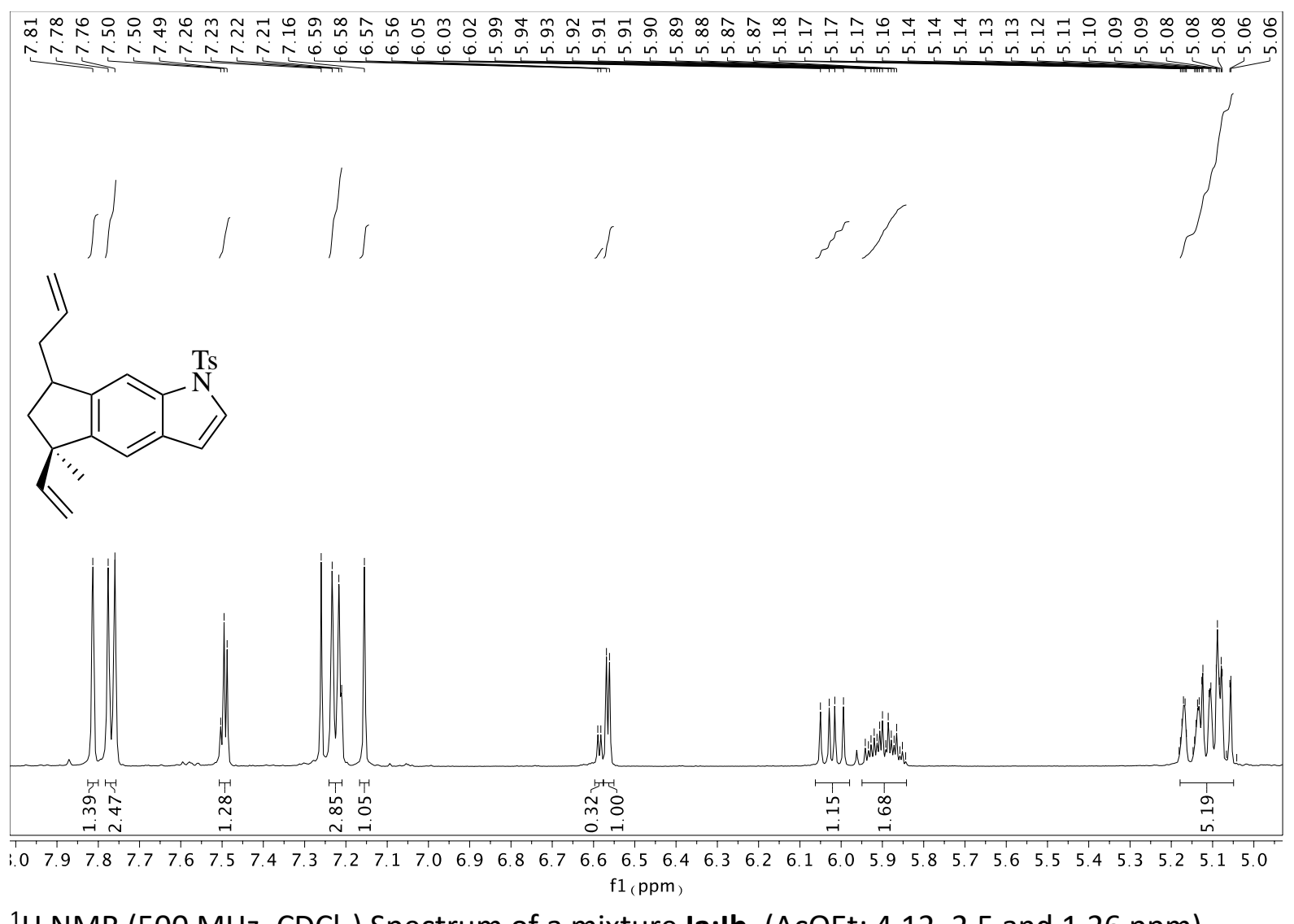

${ }^{1} \mathrm{H} \mathrm{NMR}\left(500 \mathrm{MHz}, \mathrm{CDCl}_{3}\right.$ ) Spectrum of a mixture la:Ib. (AcOEt: 4.12, 2.5 and $1.26 \mathrm{ppm}$ ). 

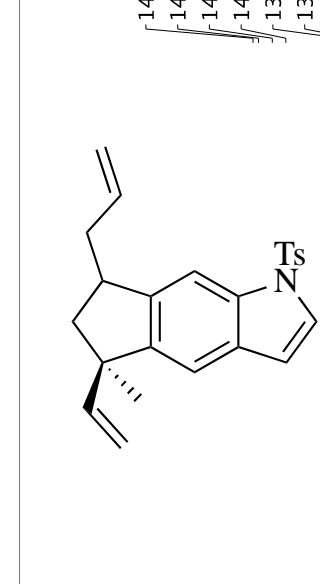

$$
170
$$

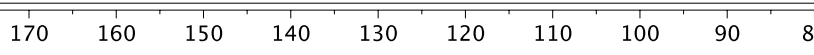

$\left.{ }^{13} \mathrm{C} \mathrm{NMR} \mathrm{(126} \mathrm{MHz,} \mathrm{CDCl}_{3}\right)$ Spectrum of a mixture la:lb.

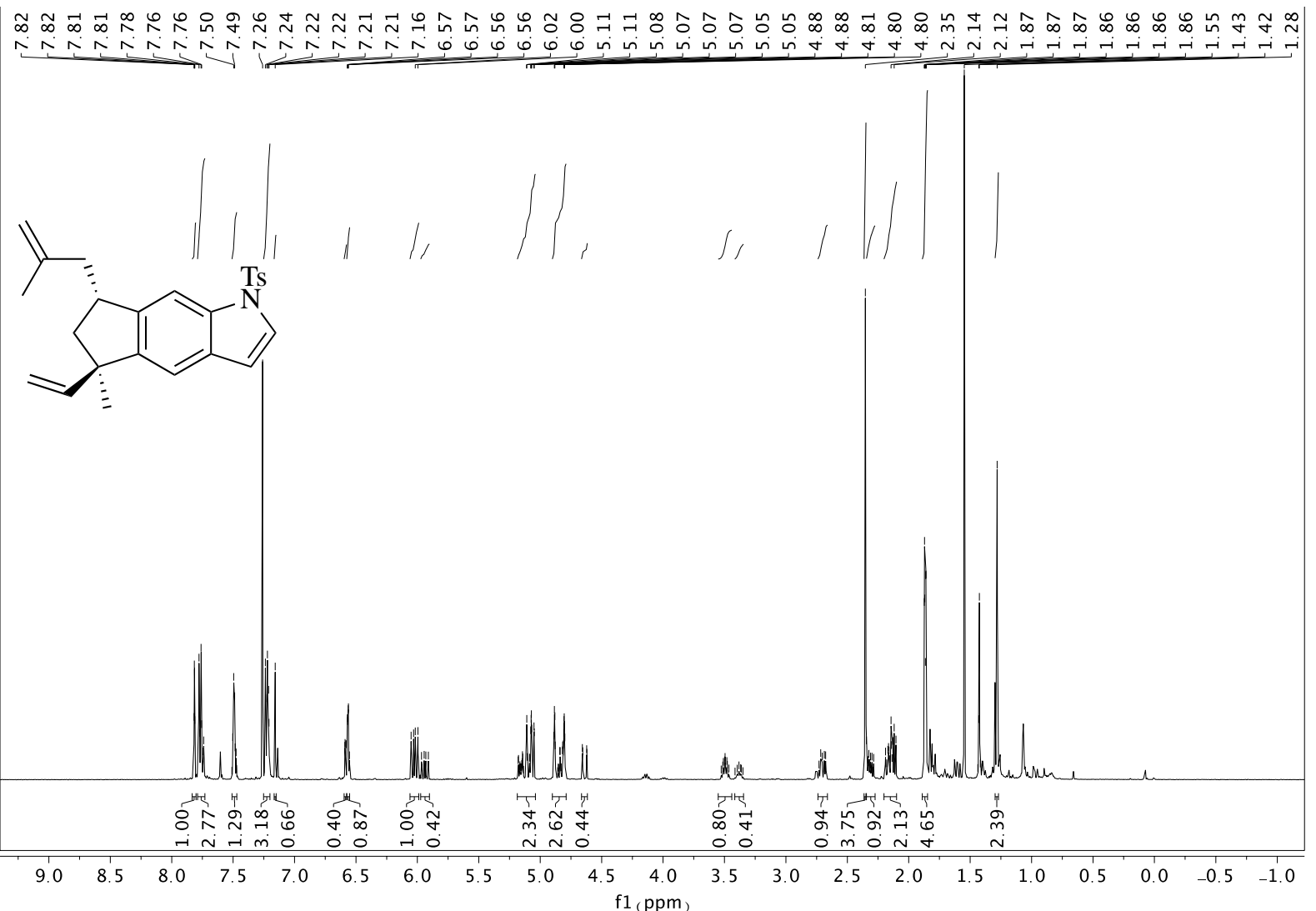

${ }^{1} \mathrm{H}$ NMR $\left(500 \mathrm{MHz}, \mathrm{CDCl}_{3}\right)$ Spectrum of a mixture (5R,7S)-14a:(5R,7R)-14b (1:2 ratio). (residual hexanes grease: 1.26 and $0.8 \mathrm{ppm}$; residual water: $1.55 \mathrm{ppm}$ ). 


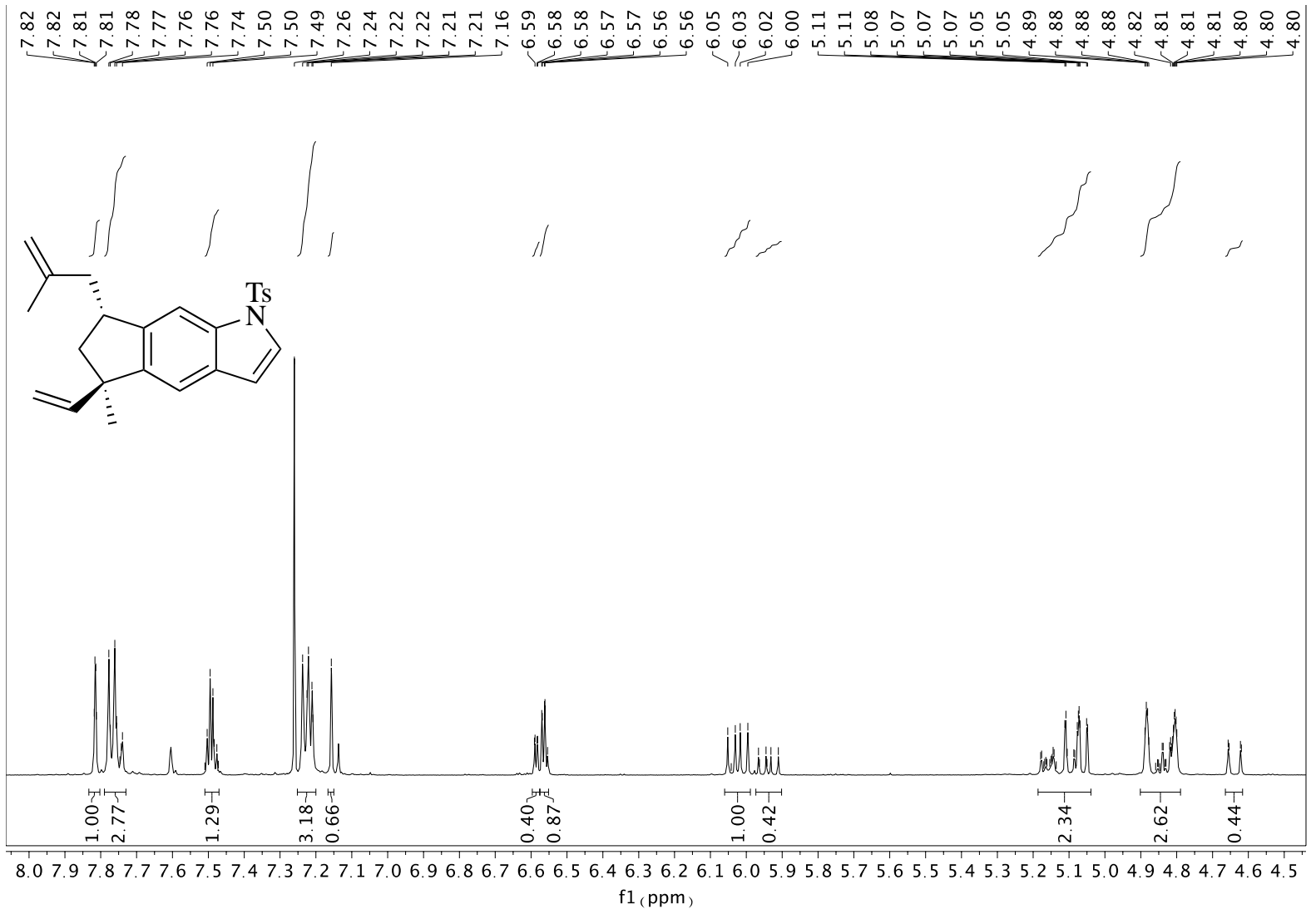

${ }^{1} \mathrm{H}$ NMR $\left(500 \mathrm{MHz}, \mathrm{CDCl}_{3}\right.$ ) Spectrum of a mixture $(5 R, 7 S)-14 \mathbf{a}:(5 R, 7 R)-14 \mathbf{b}$ (1:2 ratio).

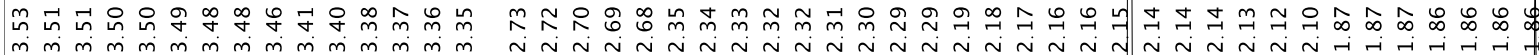

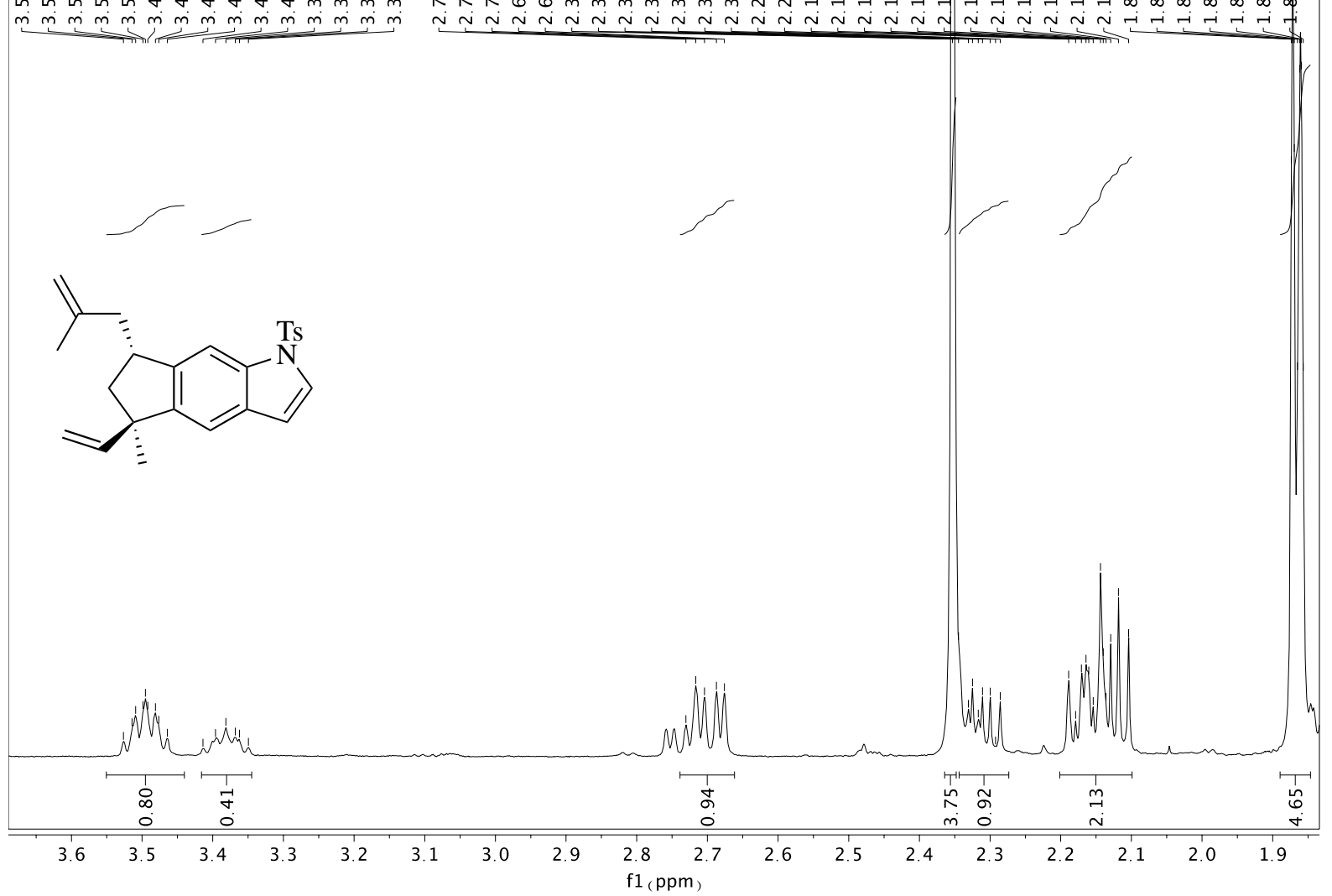


${ }^{1} \mathrm{H}$ NMR $\left(500 \mathrm{MHz}, \mathrm{CDCl}_{3}\right)$ Spectrum of a mixture $(5 R, 7 S)-14 \mathrm{a}:(5 R, 7 R)-14 \mathrm{~b}$ (1:2 ratio).

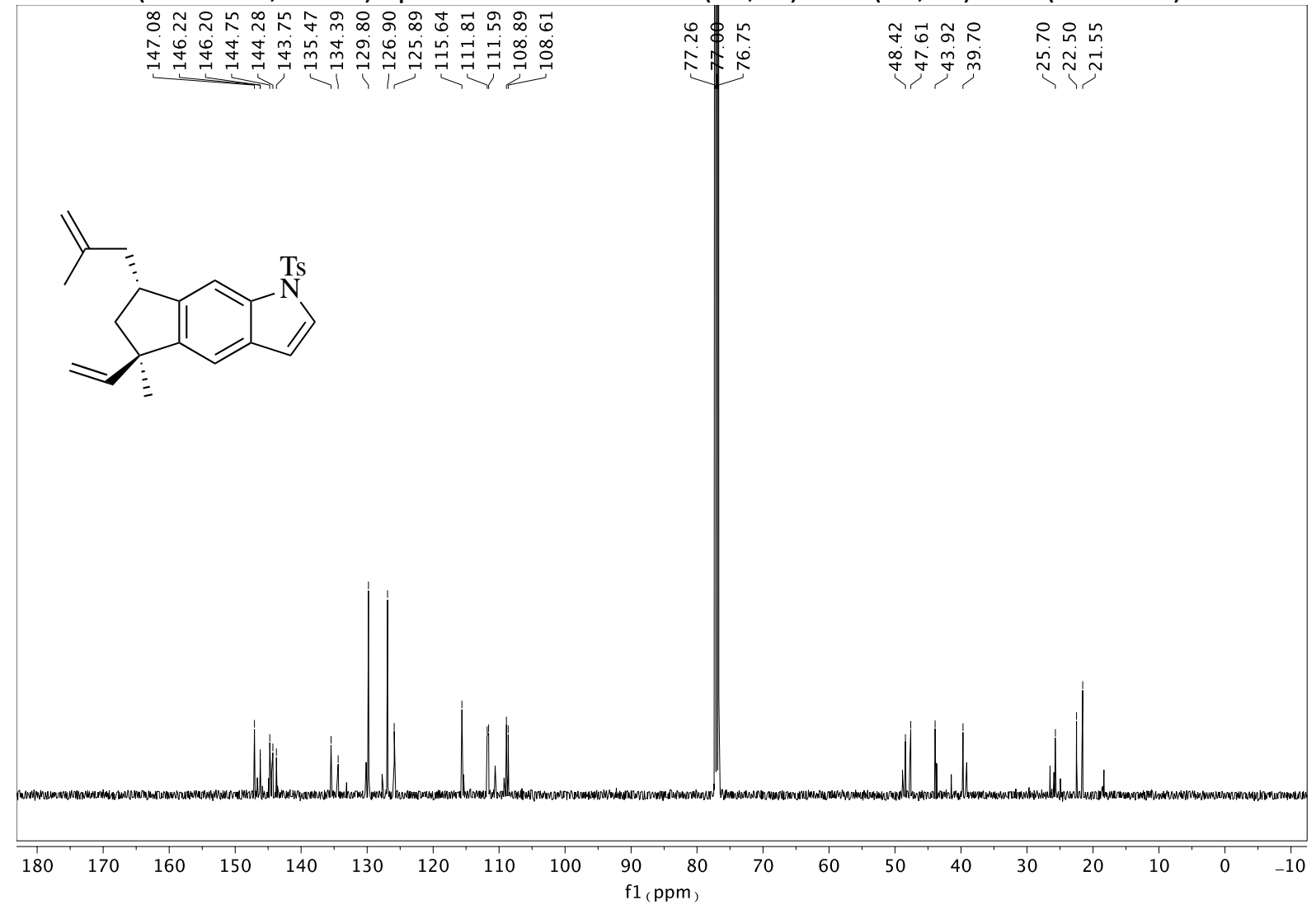

${ }^{13} \mathrm{C}$ NMR (126 MHz, CDCl 3 ) Spectrum of a mixture (5R,7S)-14a:(5R,7R)-14b (1:2 ratio).

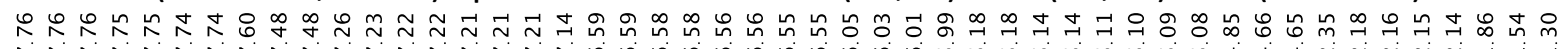

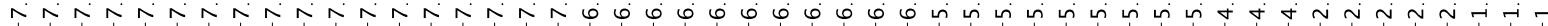

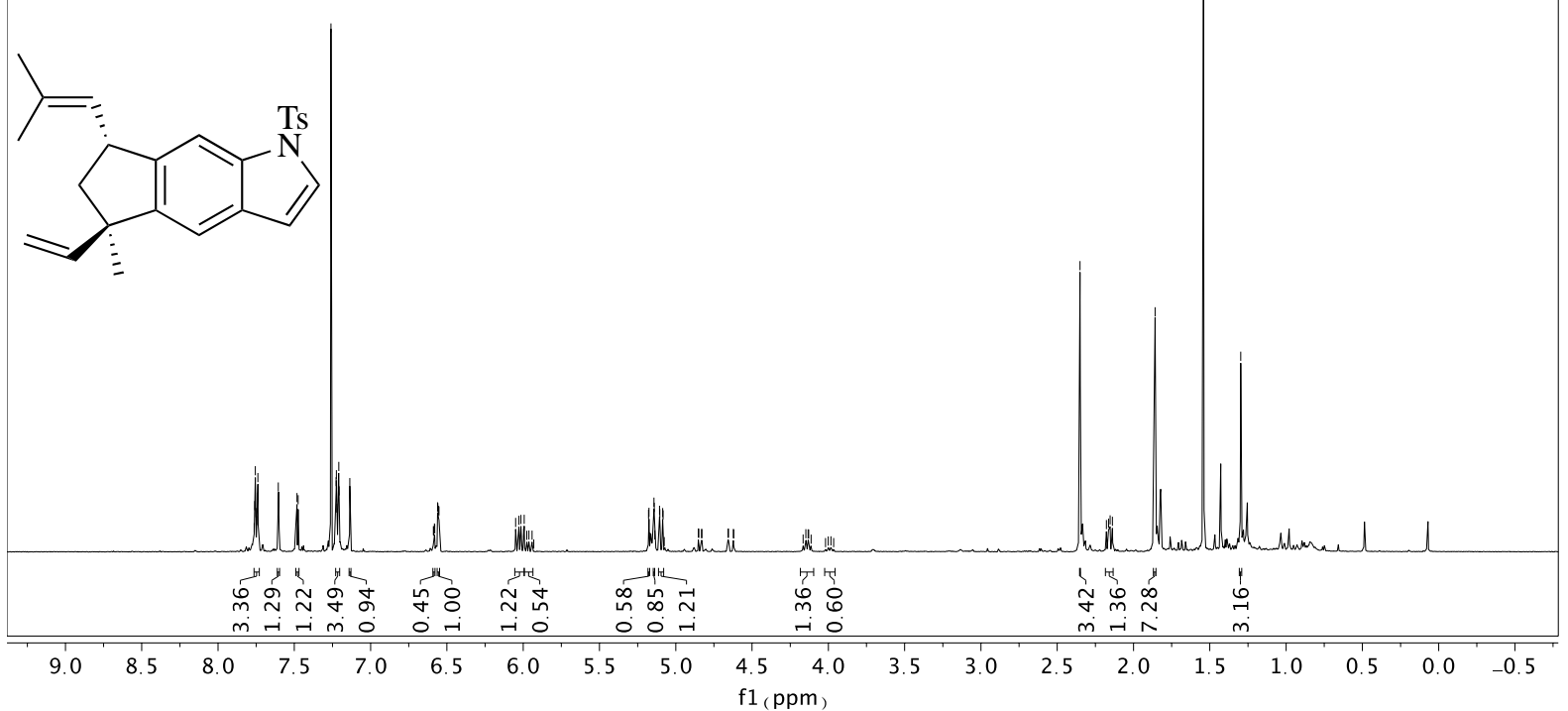

${ }^{1} \mathrm{H} \mathrm{NMR}\left(500 \mathrm{MHz}, \mathrm{CDCl}_{3}\right)$ Spectrum of a mixture (5R,7R)-18a:(5R,7S)-18b (1:2 ratio). (Obs.: spectrum with traces of starting material). 

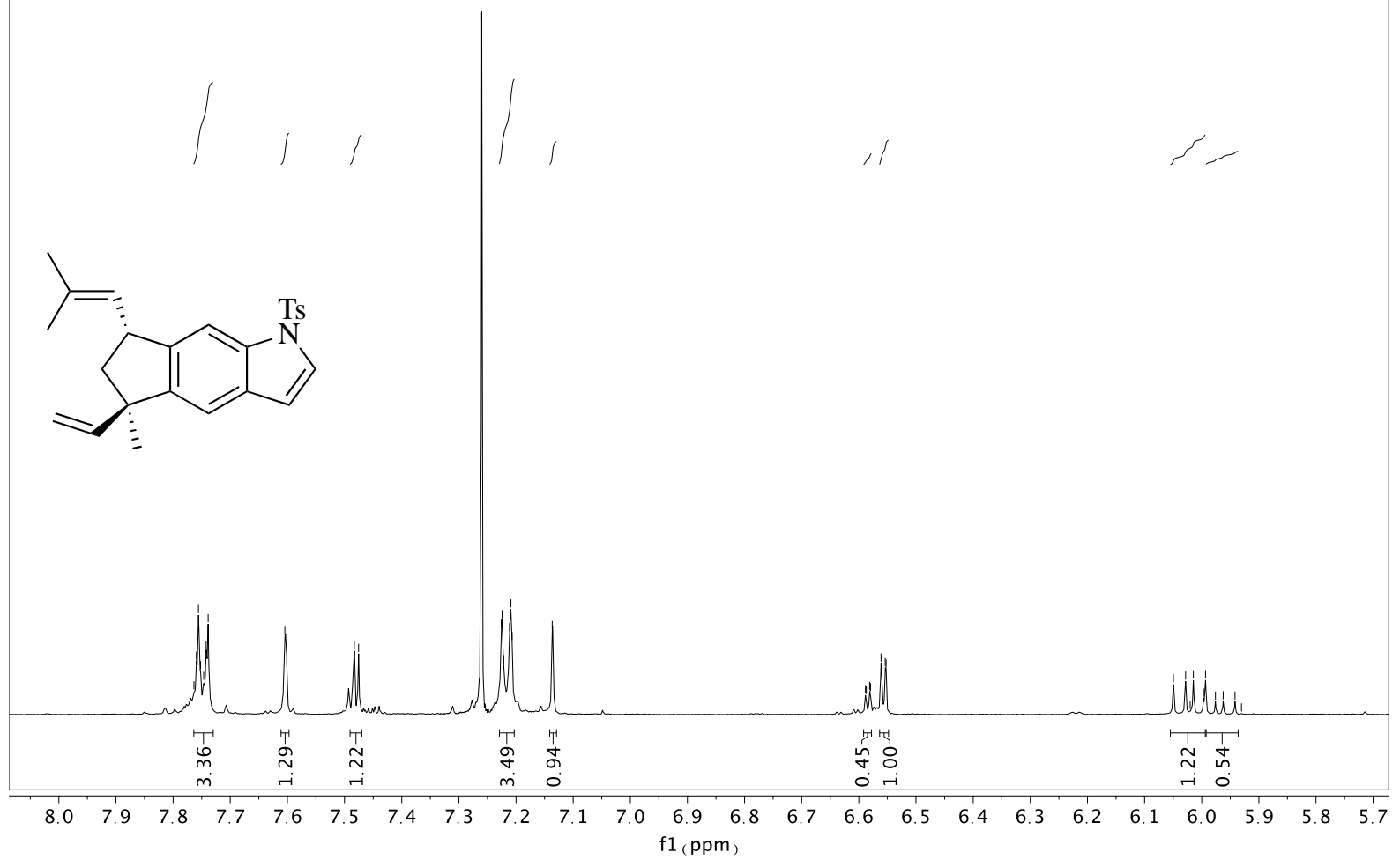

${ }^{1} \mathrm{H}$ NMR $\left(500 \mathrm{MHz}, \mathrm{CDCl}_{3}\right)$ Spectrum of a mixture $(5 R, 7 R)-\mathbf{1 8 a}:(5 R, 7 S)-\mathbf{1 8 b}$ (1:2 ratio).

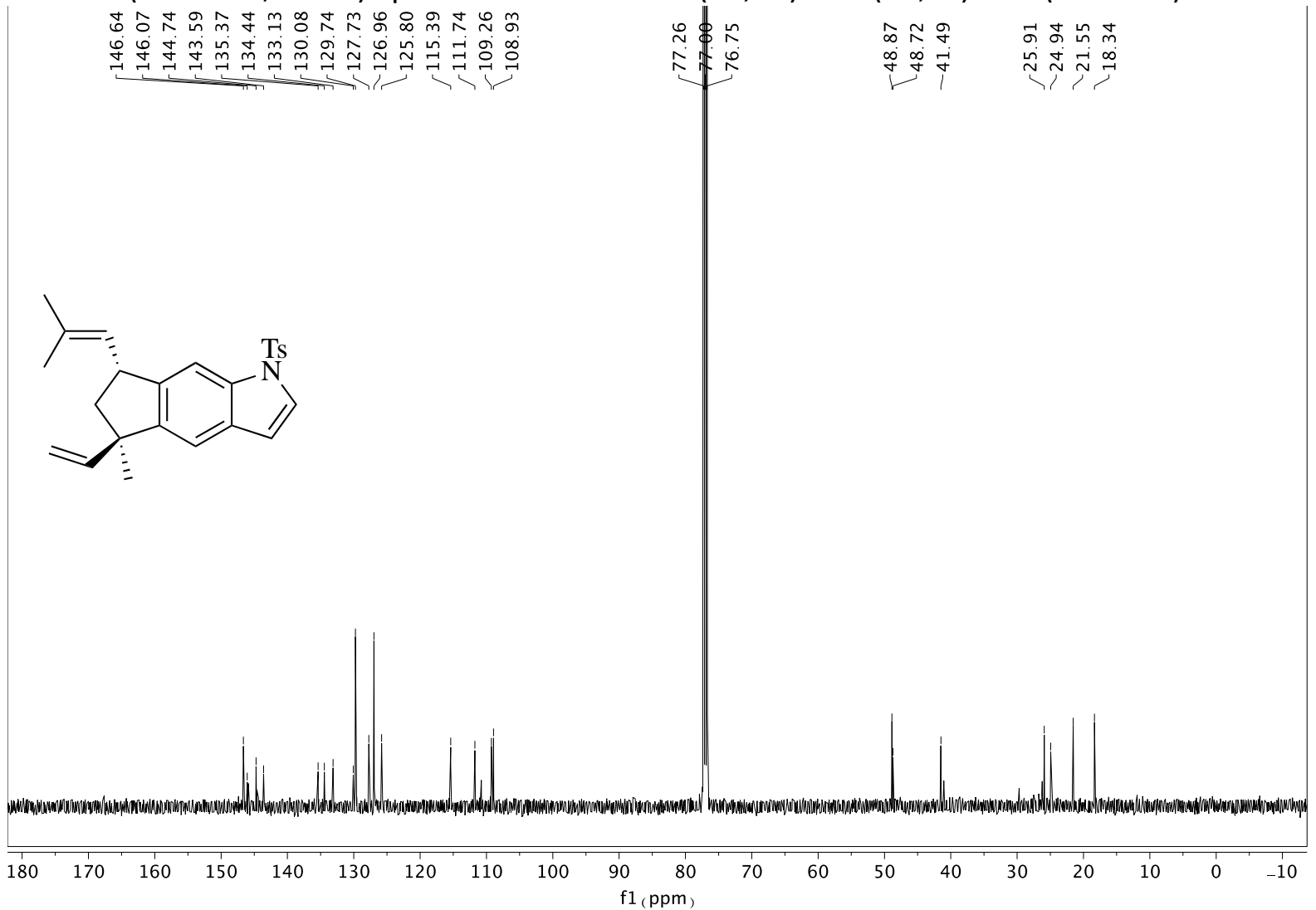

${ }^{13} \mathrm{C} \mathrm{NMR}\left(126 \mathrm{MHz}, \mathrm{CDCl}_{3}\right)$ Spectrum of a mixture $(5 R, 7 R)-\mathbf{1 8 a}:(5 R, 7 S)-\mathbf{1 8 b}$ (1:2 ratio). 


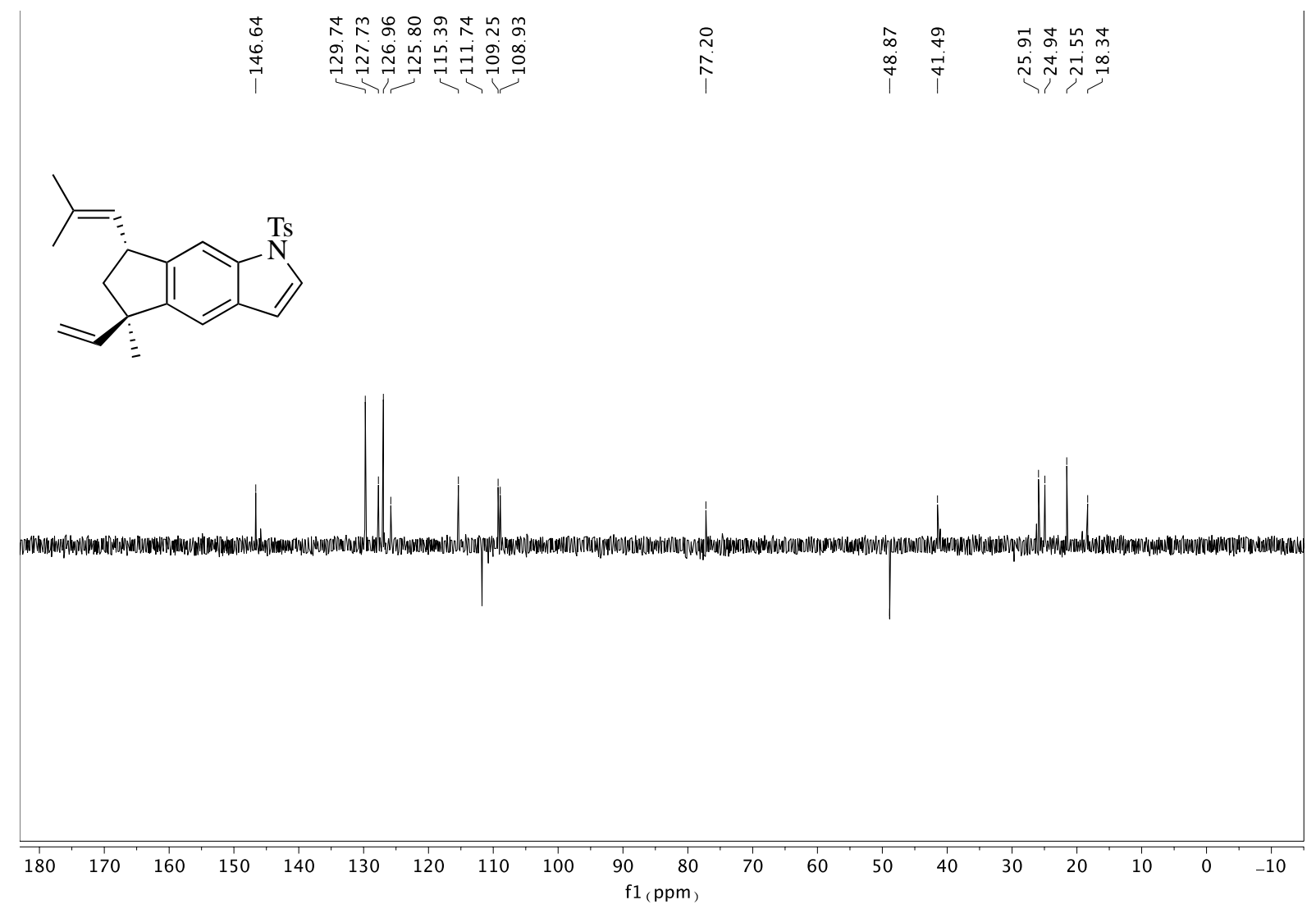

DEPT135 (126 MHz, CDCl 3 ) Spectrum of a mixture (5R,7R)-18a:(5R,7S)-18b (1:2 ratio).

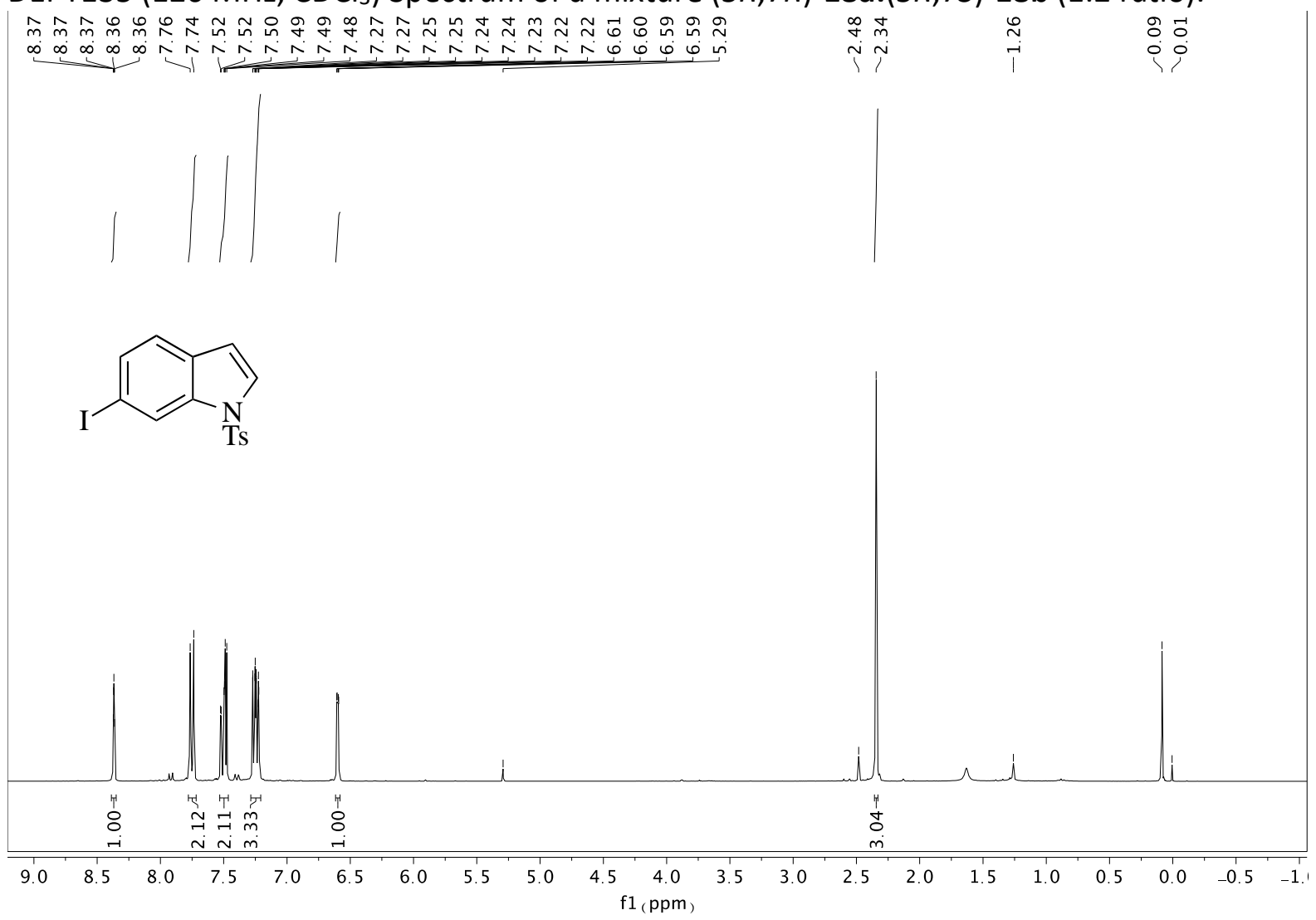

${ }^{1} \mathrm{H}$ NMR (300 MHz, $\mathrm{CDCl}_{3}$ ) Spectrum of compound 17. (DCM: $5.27 \mathrm{ppm}$ and TsCl impurity). 


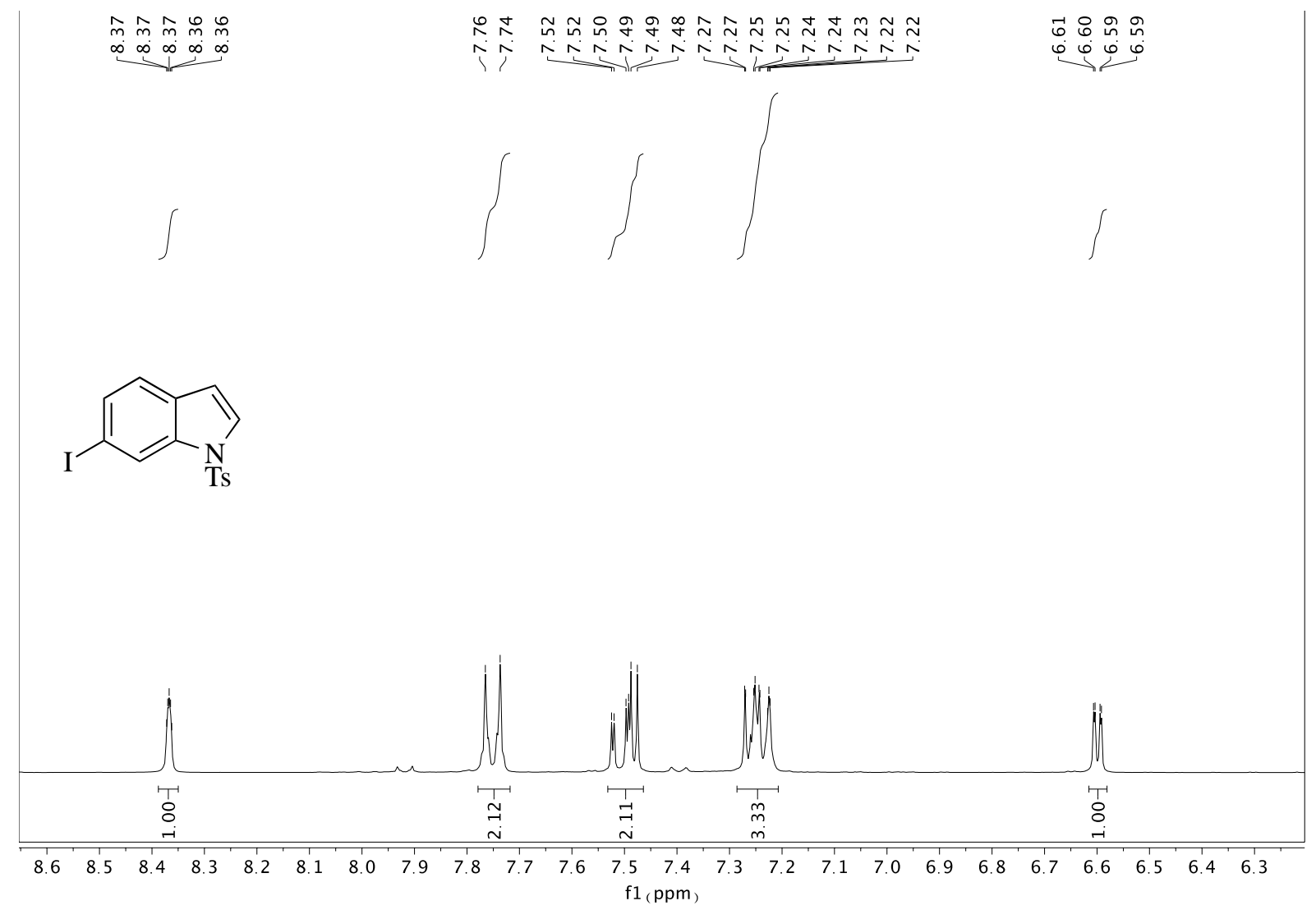

${ }^{1} \mathrm{H}$ NMR (300 MHz, CDCl ) Spectrum of compound 17.

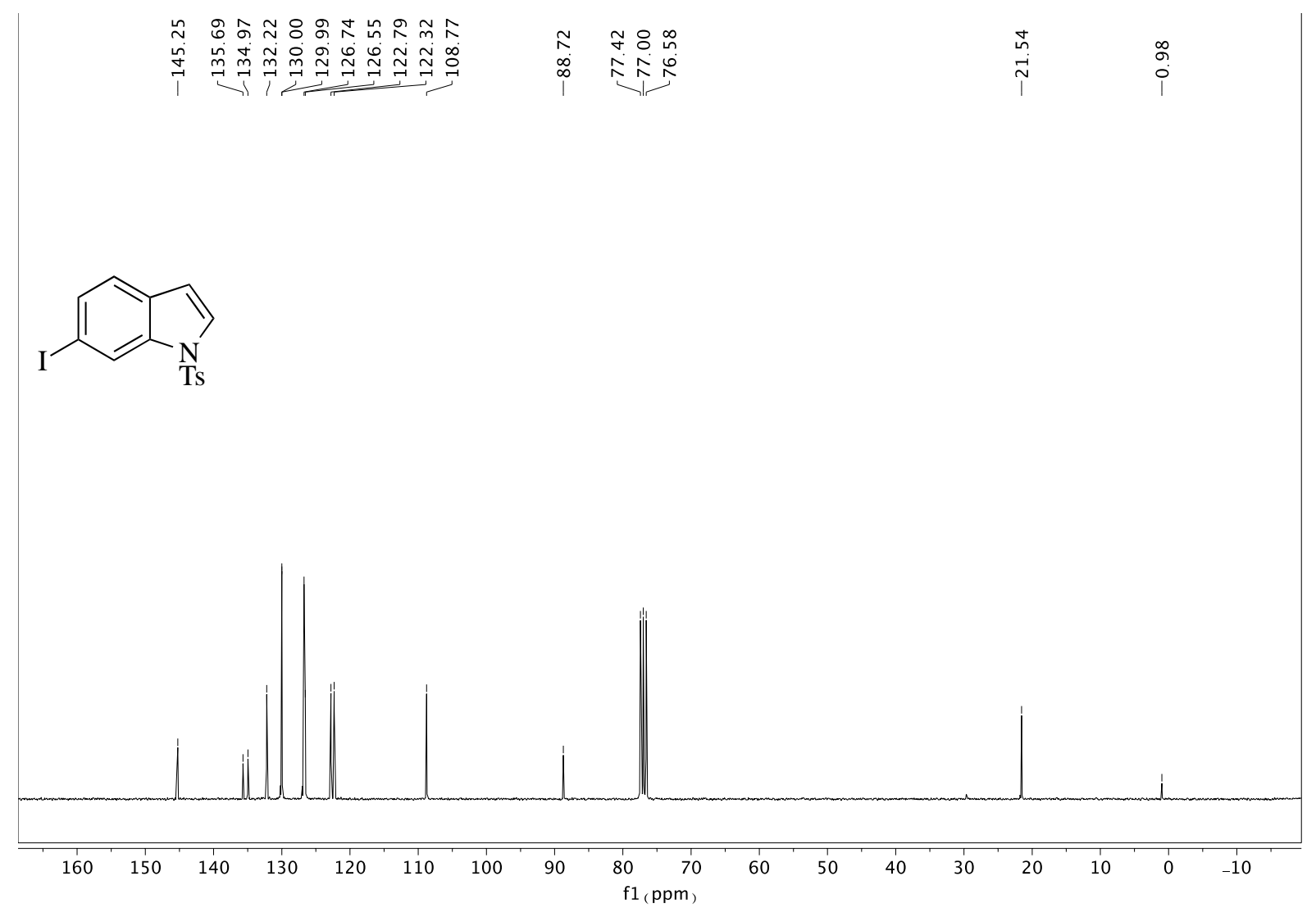

${ }^{13} \mathrm{C} \mathrm{NMR}\left(75 \mathrm{MHz}, \mathrm{CDCl}_{3}\right.$ ) Spectrum of Compound 17. 


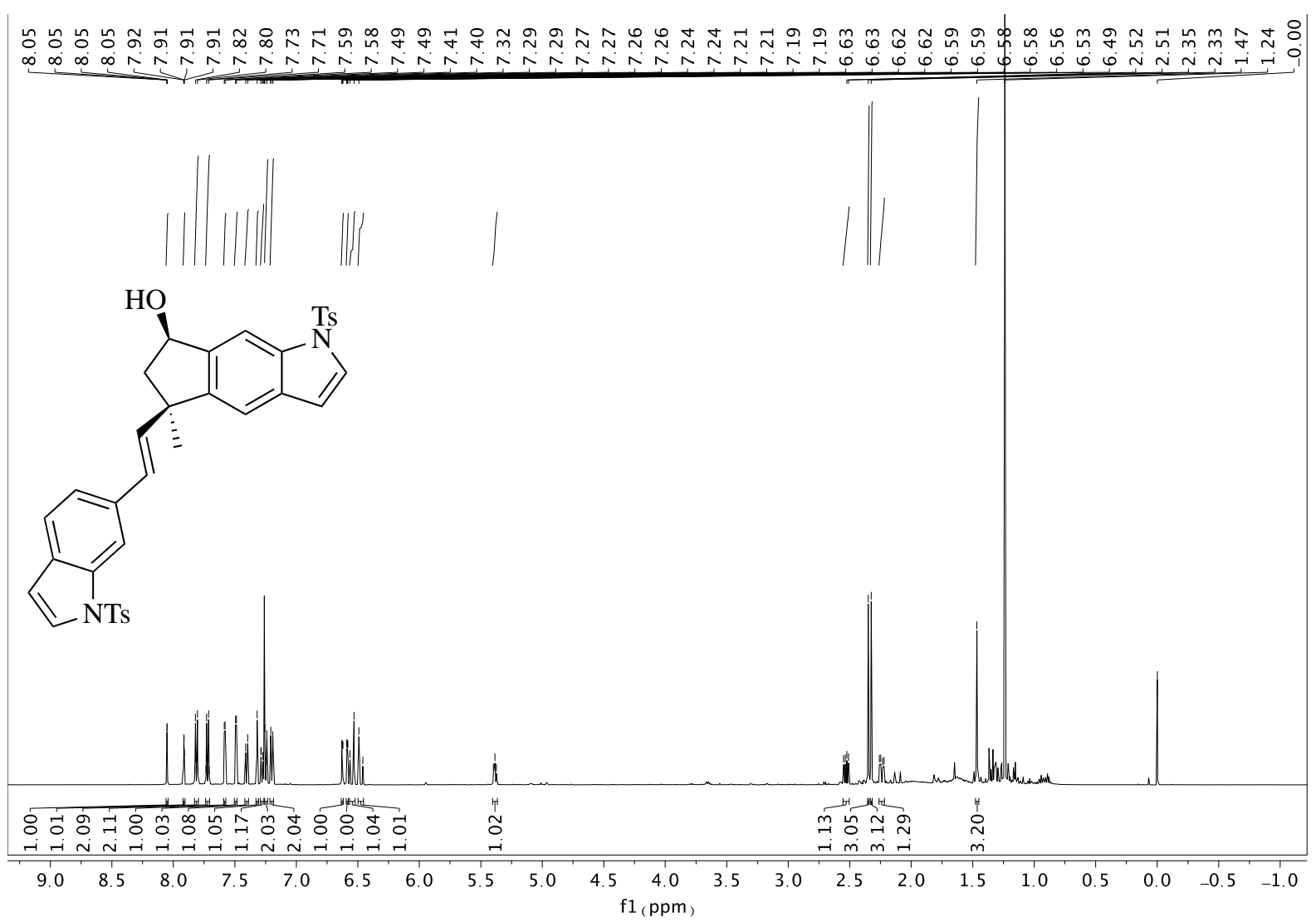

${ }^{1} \mathrm{H} \mathrm{NMR}\left(500 \mathrm{MHz}, \mathrm{CDCl}_{3}\right.$ ) Spectrum of compound 15.

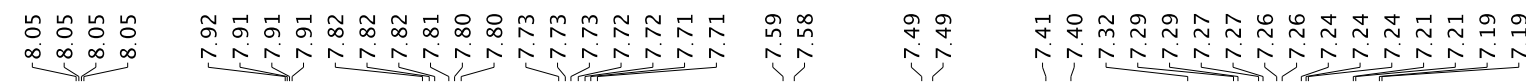

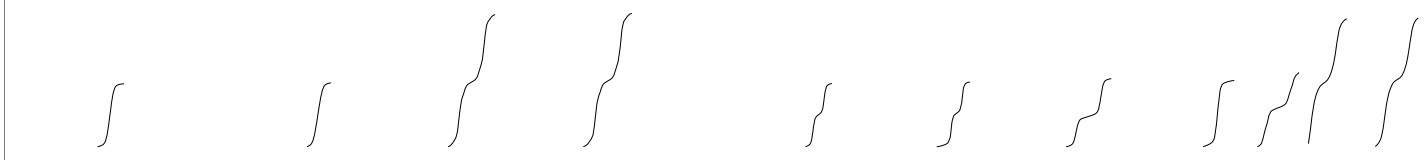<smiles>OC1C[C@H](C=Cc2ccc3cc[nH]c3c2)c2cc3cc[n-]c3cc21</smiles>

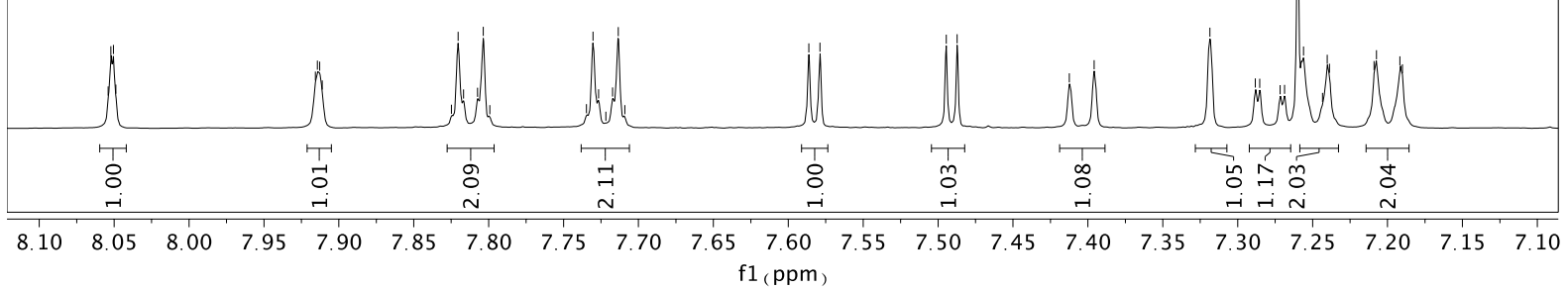

${ }^{1} \mathrm{H} \mathrm{NMR}\left(500 \mathrm{MHz}, \mathrm{CDCl}_{3}\right.$ ) Spectrum of compound 15. 


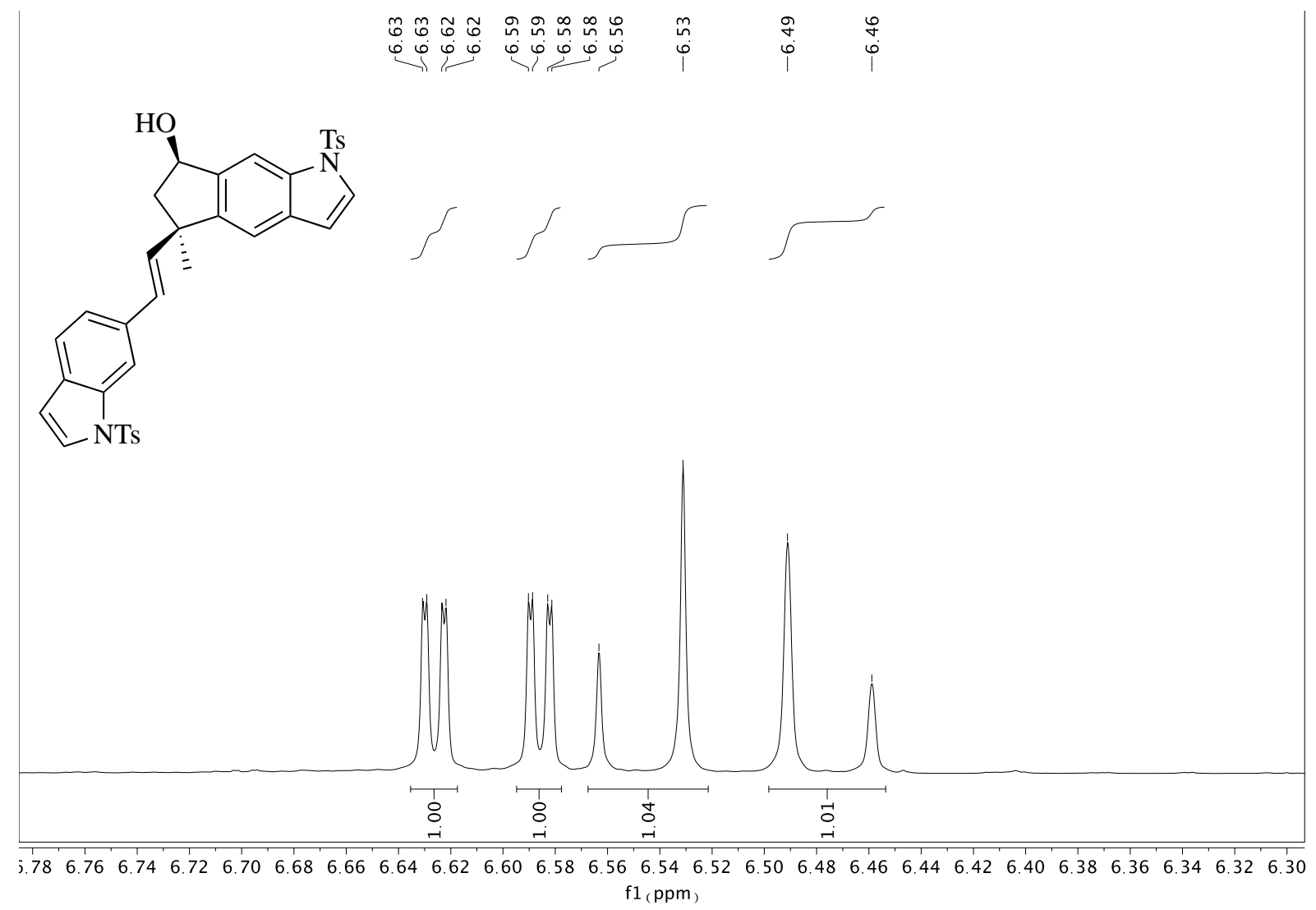

${ }^{1} \mathrm{H} \mathrm{NMR}\left(500 \mathrm{MHz}, \mathrm{CDCl}_{3}\right.$ ) Spectrum of compound 15.

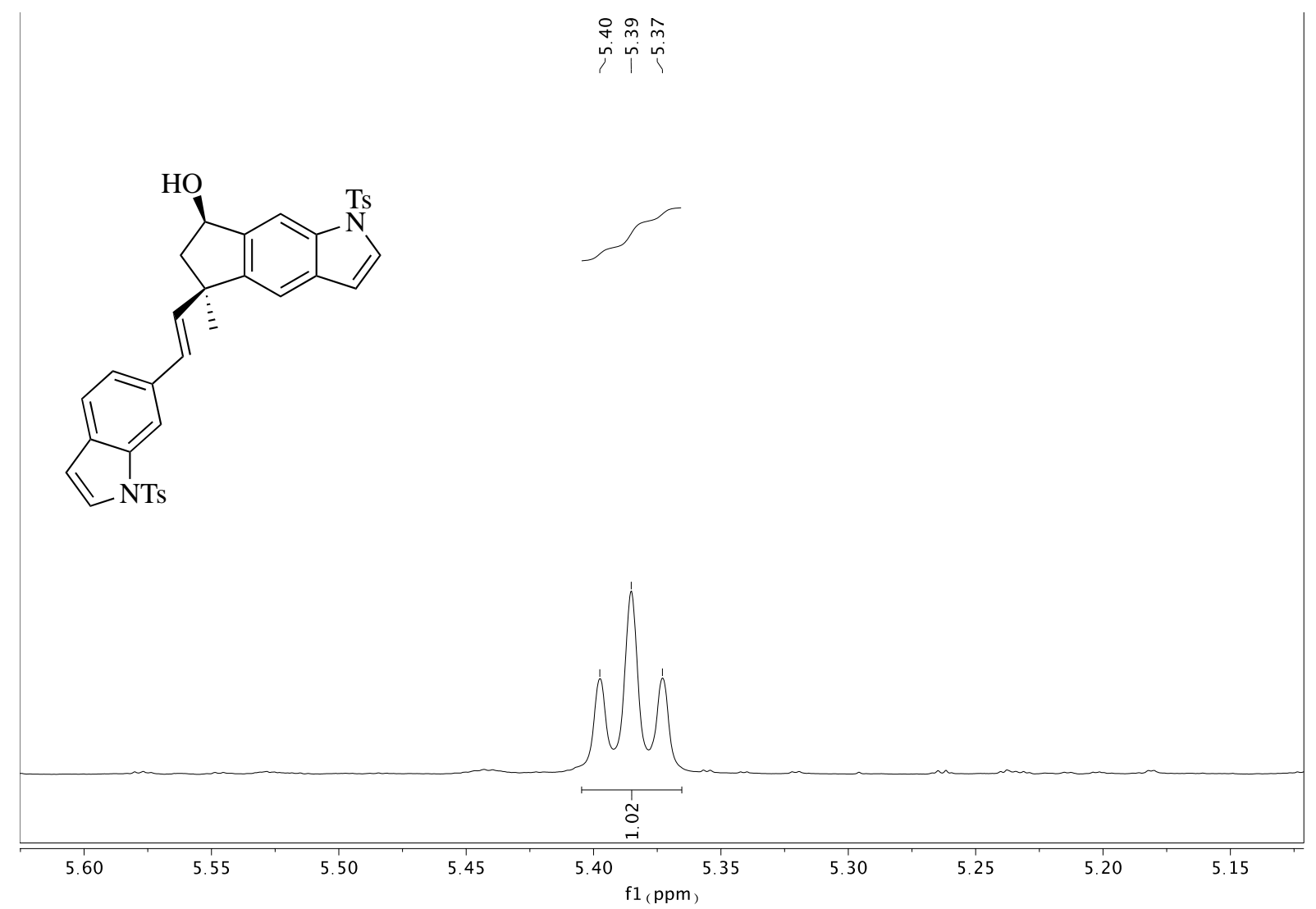

${ }^{1} \mathrm{H}$ NMR (500 MHz, CDCl ) Spectrum of compound 15. 

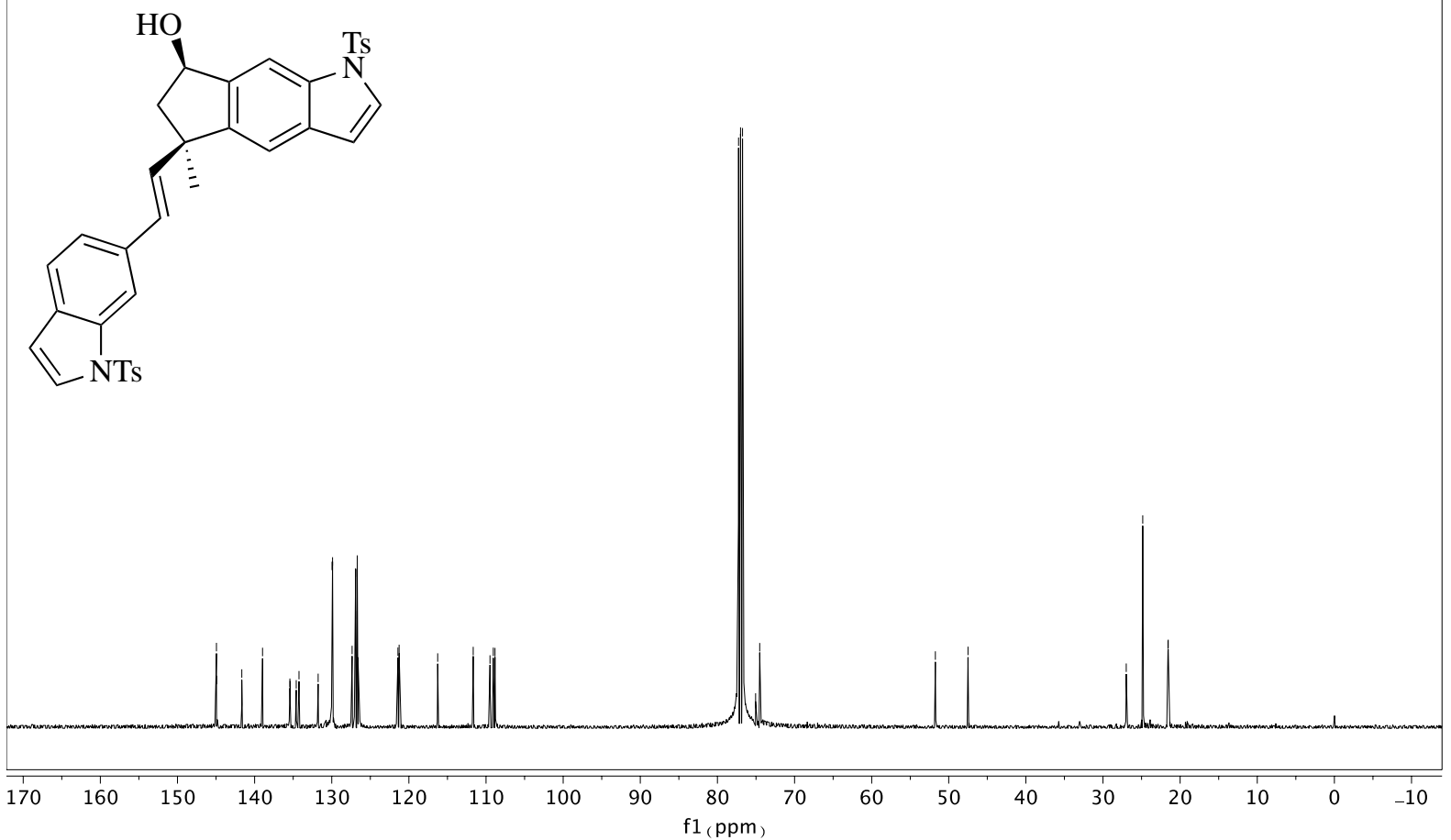

${ }^{13} \mathrm{C}$ NMR $\left(126 \mathrm{MHz}, \mathrm{CDCl}_{3}\right)$ Spectrum of Compound 15.

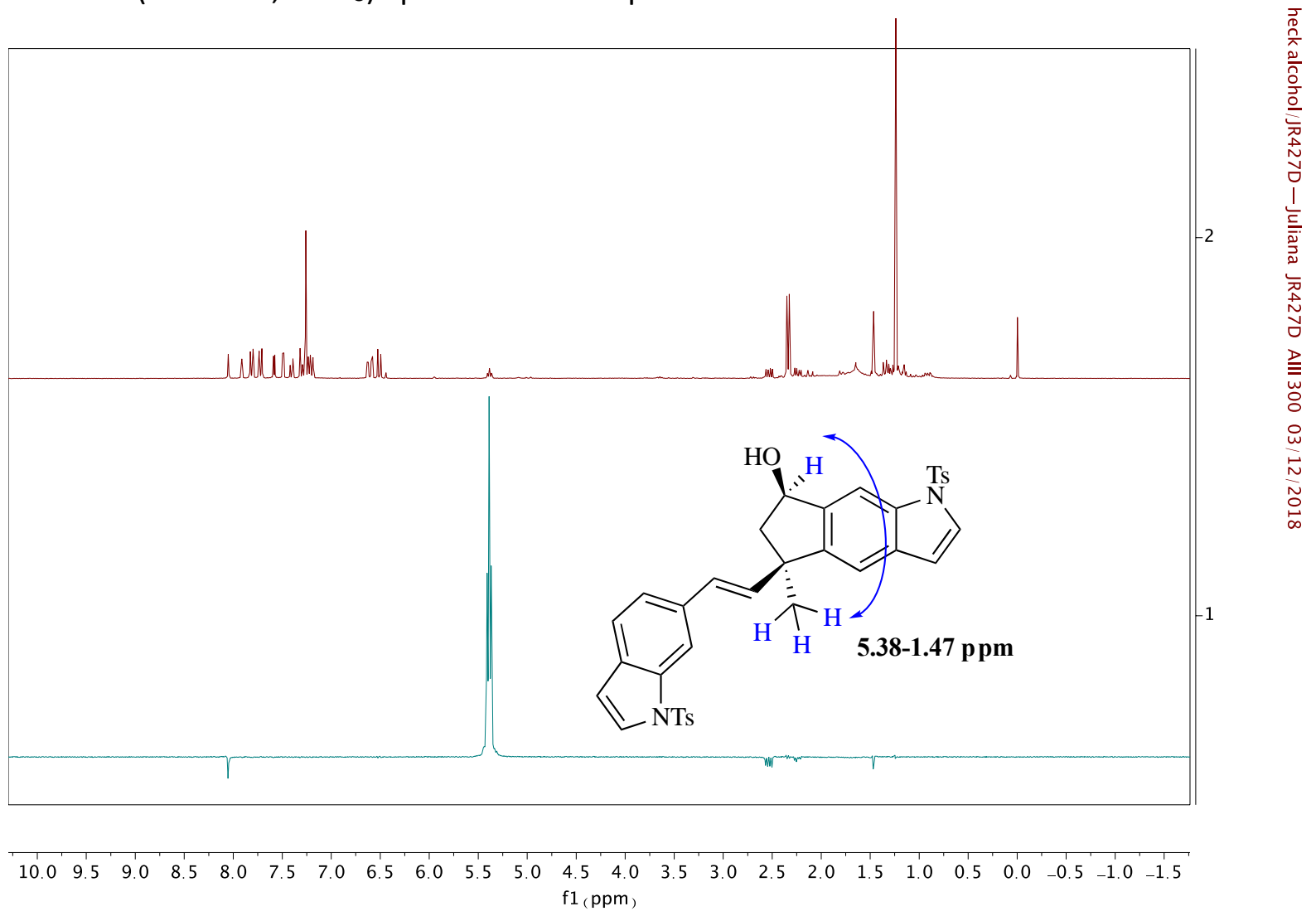

NOE 1D NMR (300 MHz, $\mathrm{CDCl}_{3}$ ) Spectrum of compound 15. (irradiation: $5.38 \mathrm{ppm}$ ). 


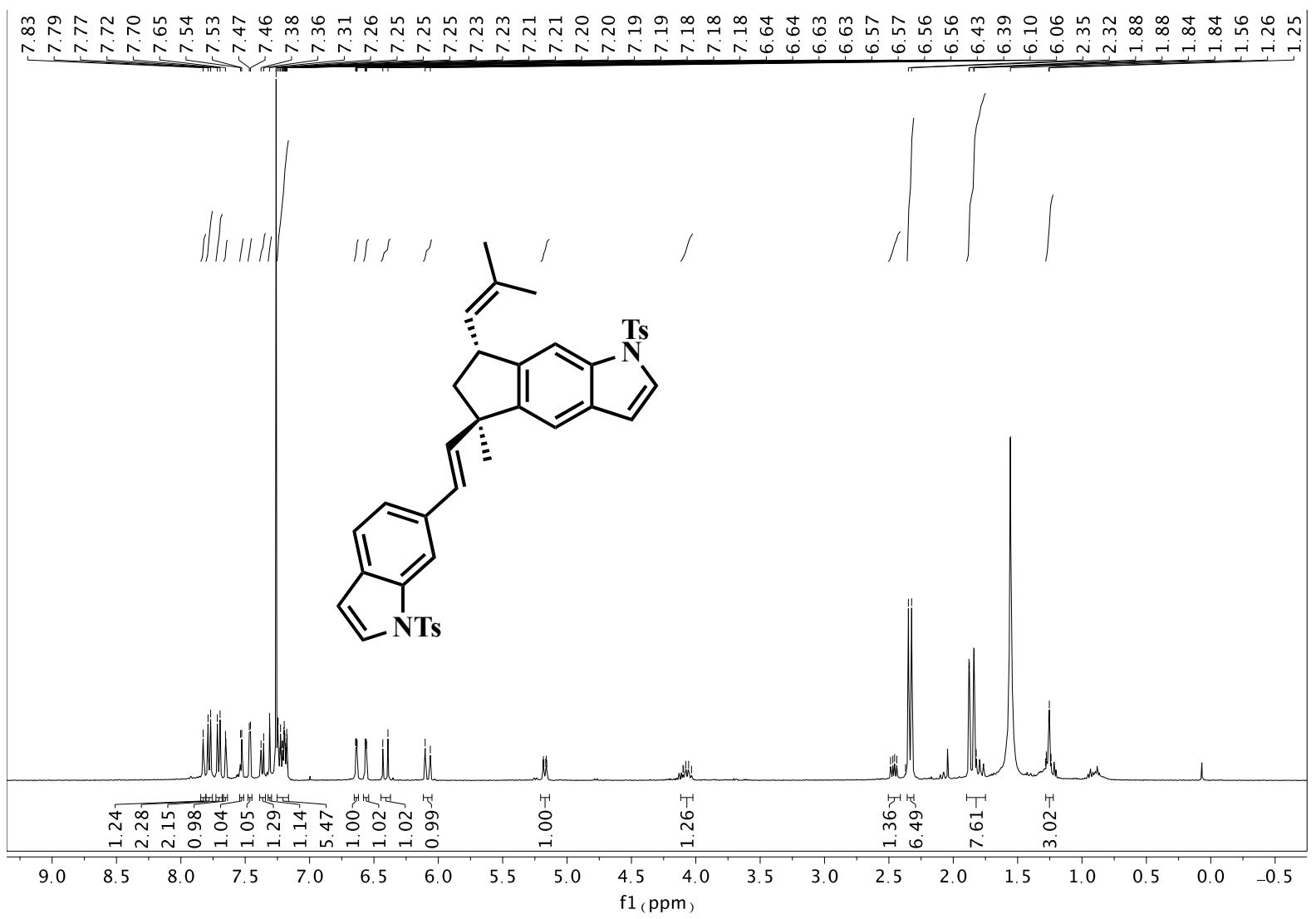

${ }^{1} \mathrm{H}$ NMR $\left(400 \mathrm{MHz}, \mathrm{CDCl}_{3}\right)$ Spectrum of $(5 R, 7 R)$-19a. (residual hexanes grease: 1.26 and 0.8 ppm; residual water: $1.56 \mathrm{ppm})$.

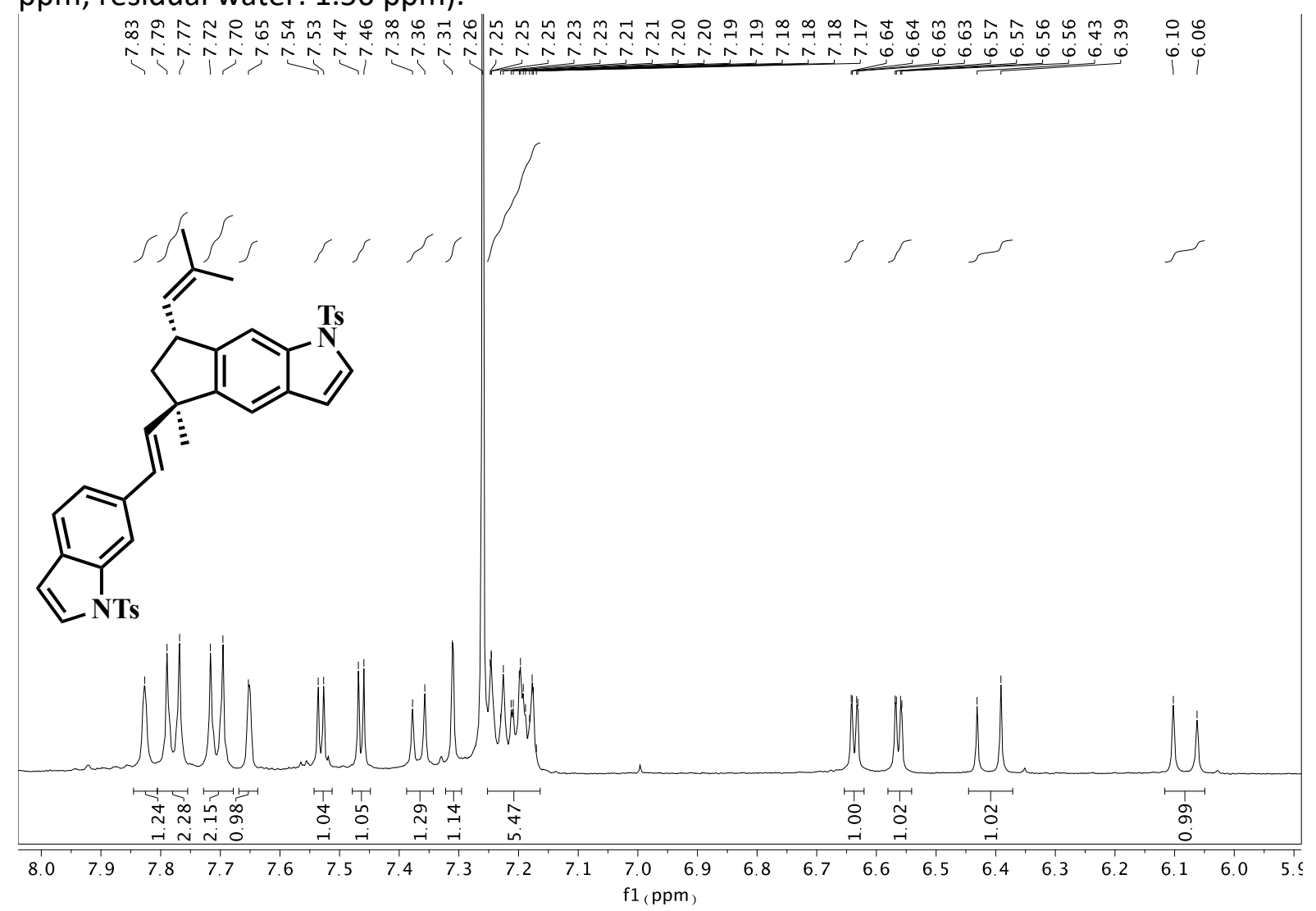

${ }^{1} \mathrm{H} \mathrm{NMR}\left(400 \mathrm{MHz}, \mathrm{CDCl}_{3}\right)$ Spectrum of $(5 R, 7 R)-19 \mathrm{a}$. 


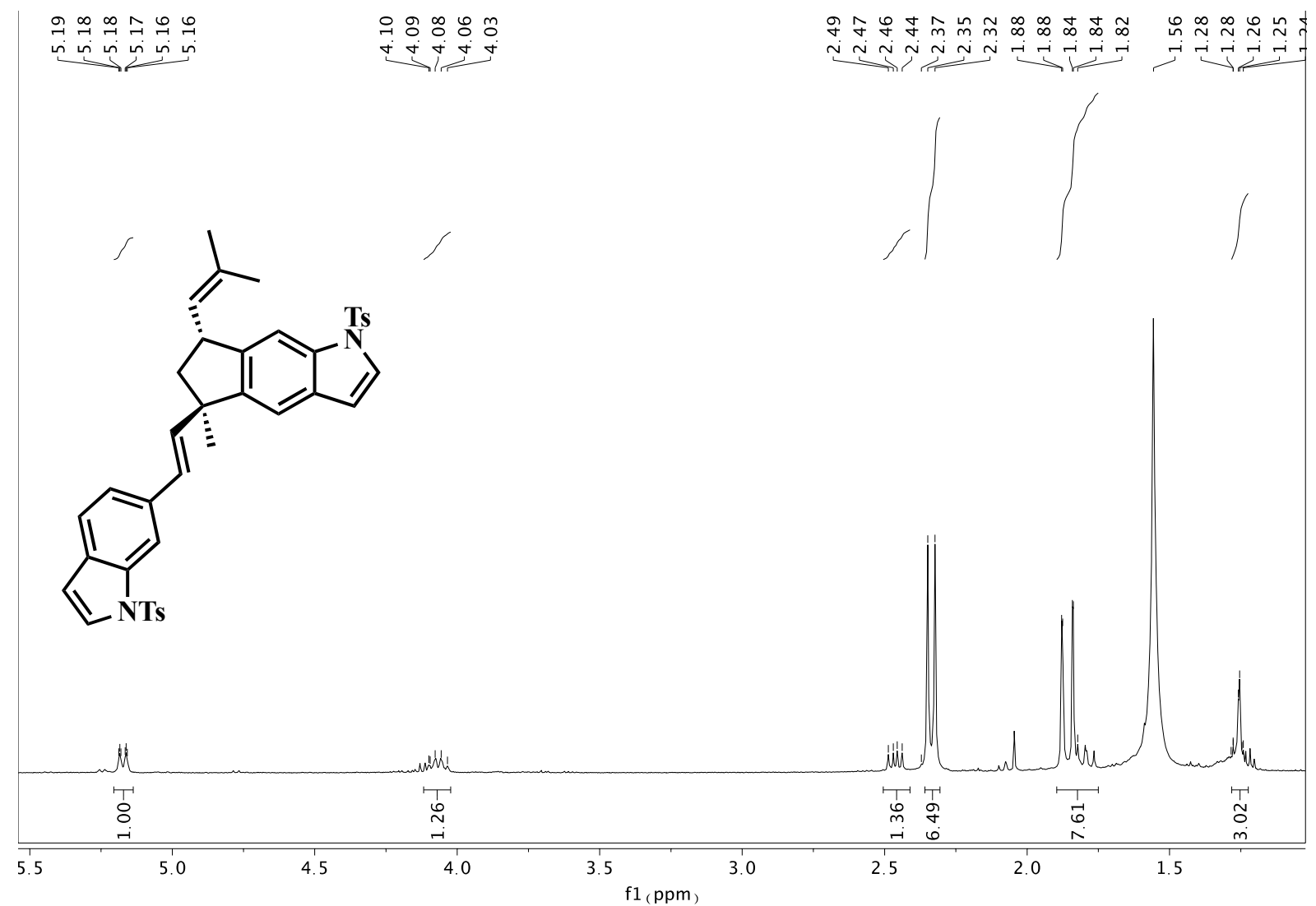

${ }^{1} \mathrm{H}$ NMR $\left(400 \mathrm{MHz}, \mathrm{CDCl}_{3}\right)$ Spectrum of $(5 R, 7 R)-19 \mathrm{a}$.

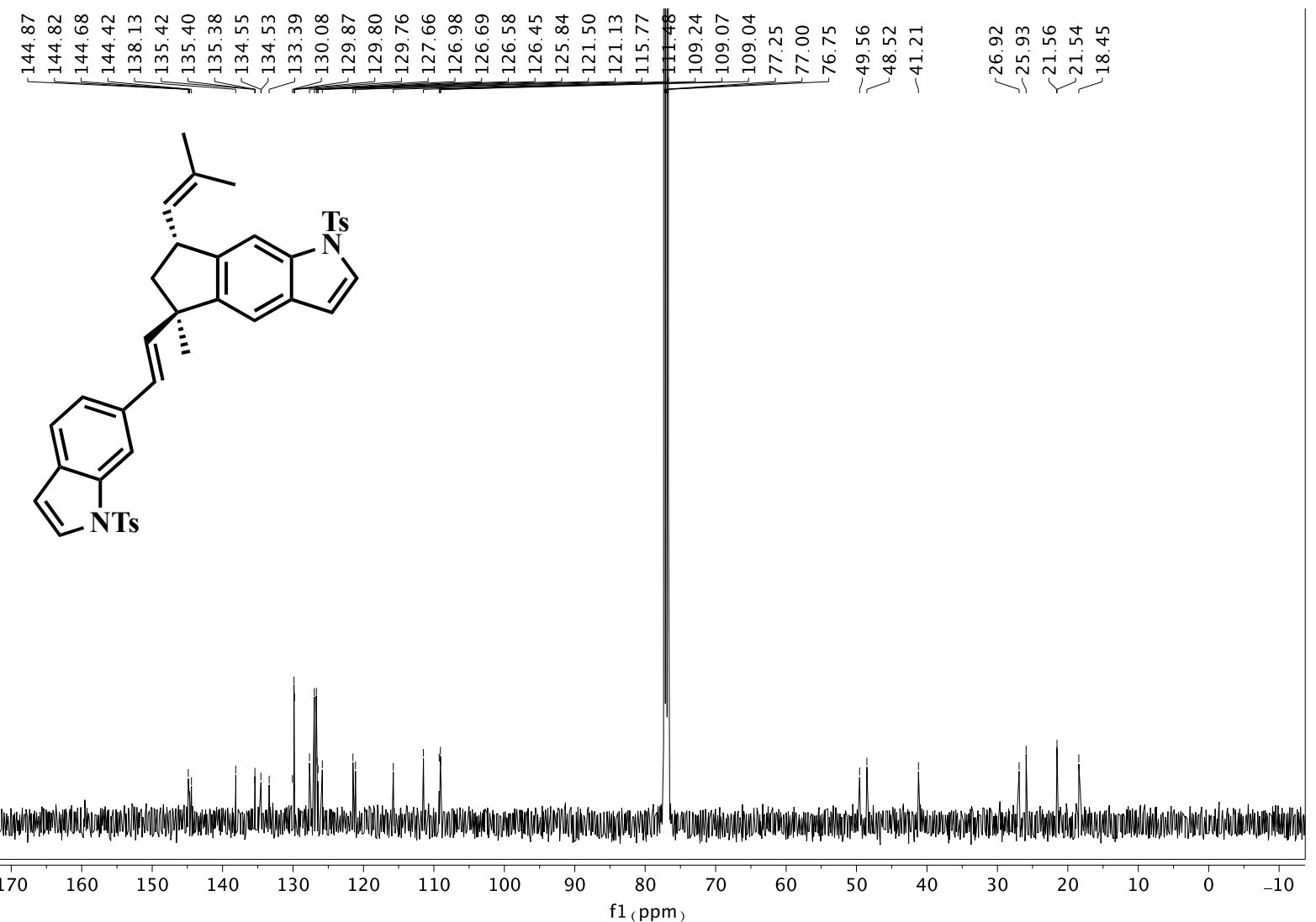

${ }^{13} \mathrm{C} \mathrm{NMR}\left(126 \mathrm{MHz}, \mathrm{CDCl}_{3}\right)$ Spectrum of $(5 R, 7 R)-19 \mathrm{a}$. 


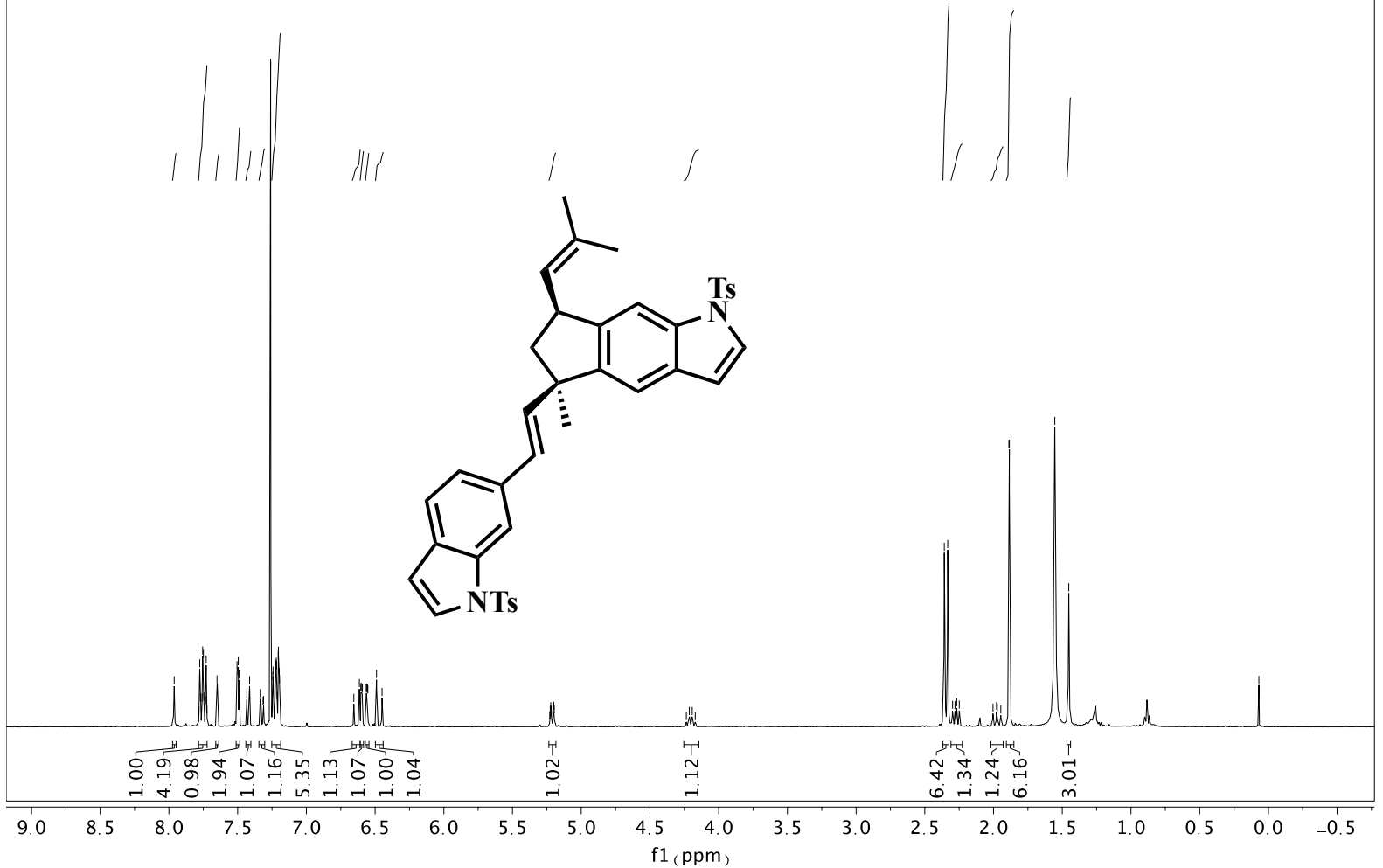

${ }^{1} \mathrm{H}$ NMR (400 MHz, $\mathrm{CDCl}_{3}$ ) Spectrum of $(5 R, 7 S)$-19b. (residual hexanes grease: 1.26 and 0.8 ppm; residual water: $1.56 \mathrm{ppm})$.

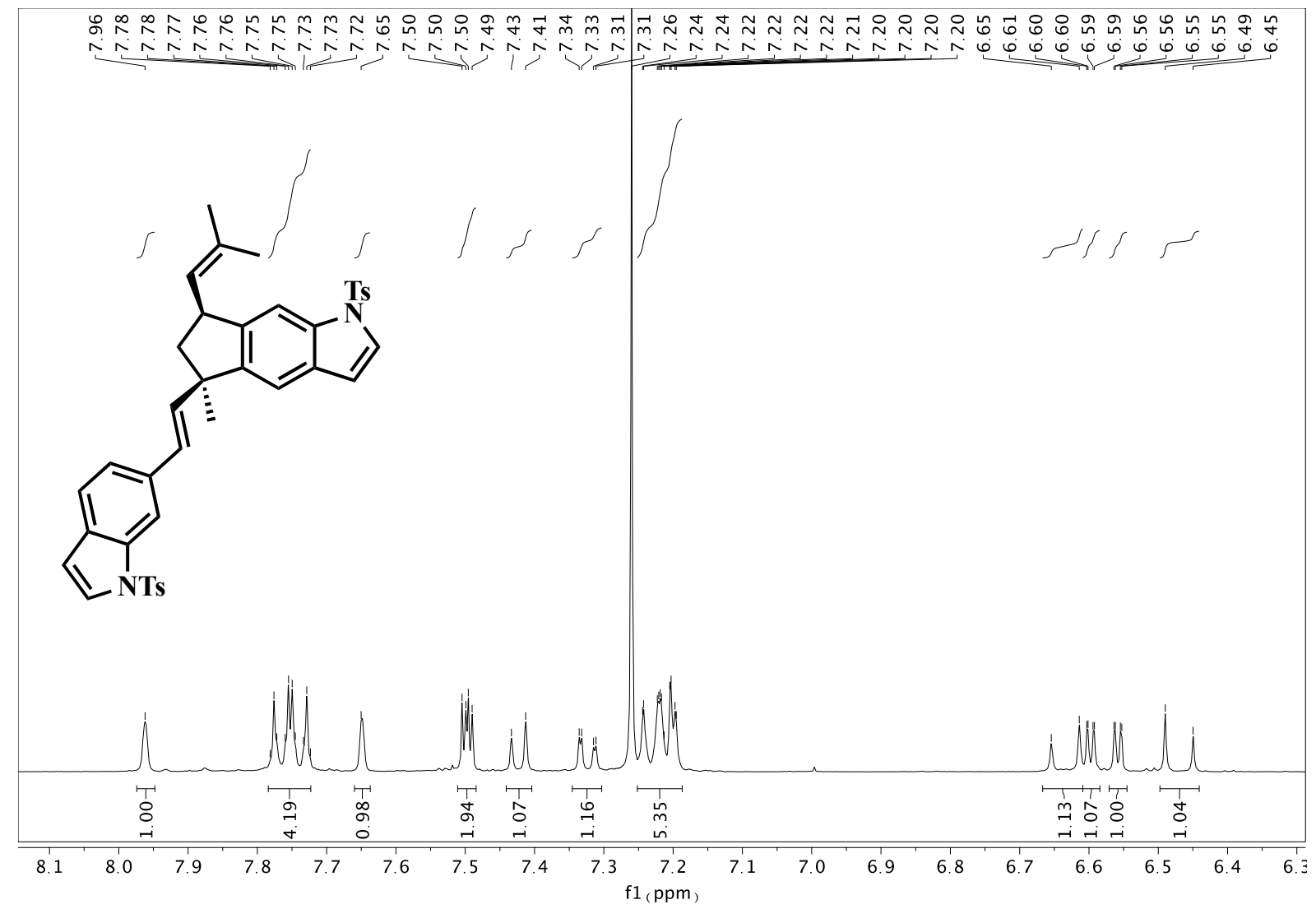

${ }^{1} \mathrm{H}$ NMR $\left(400 \mathrm{MHz}, \mathrm{CDCl}_{3}\right)$ Spectrum of $(5 R, 7 S)-\mathbf{1 9 b}$. 


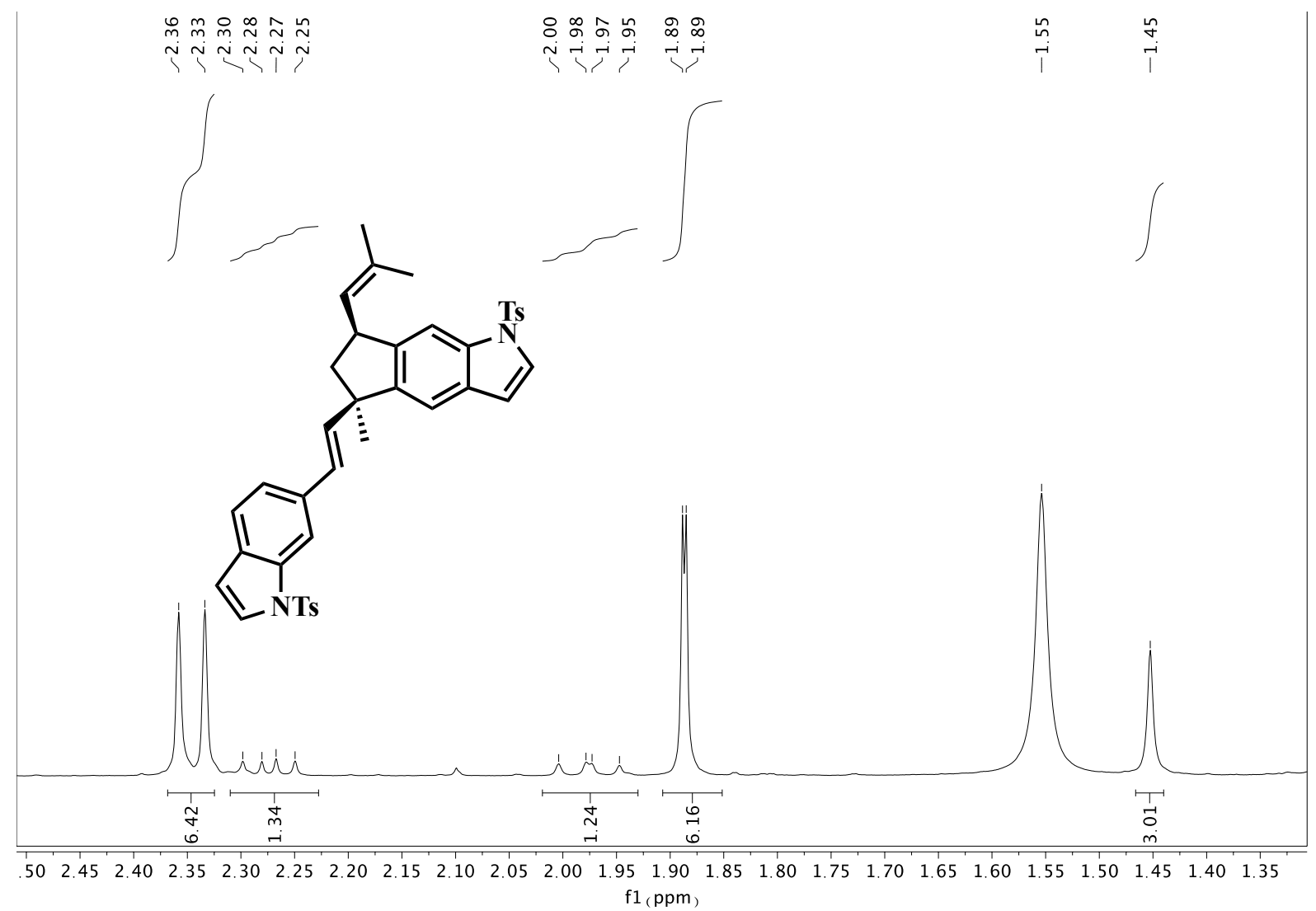

${ }^{1} \mathrm{H}$ NMR $\left(400 \mathrm{MHz}, \mathrm{CDCl}_{3}\right.$ ) Spectrum of $(5 R, 7 S)$-19b. (residual hexanes grease: 1.26 and 0.8 ppm; residual water: $1.56 \mathrm{ppm})$.

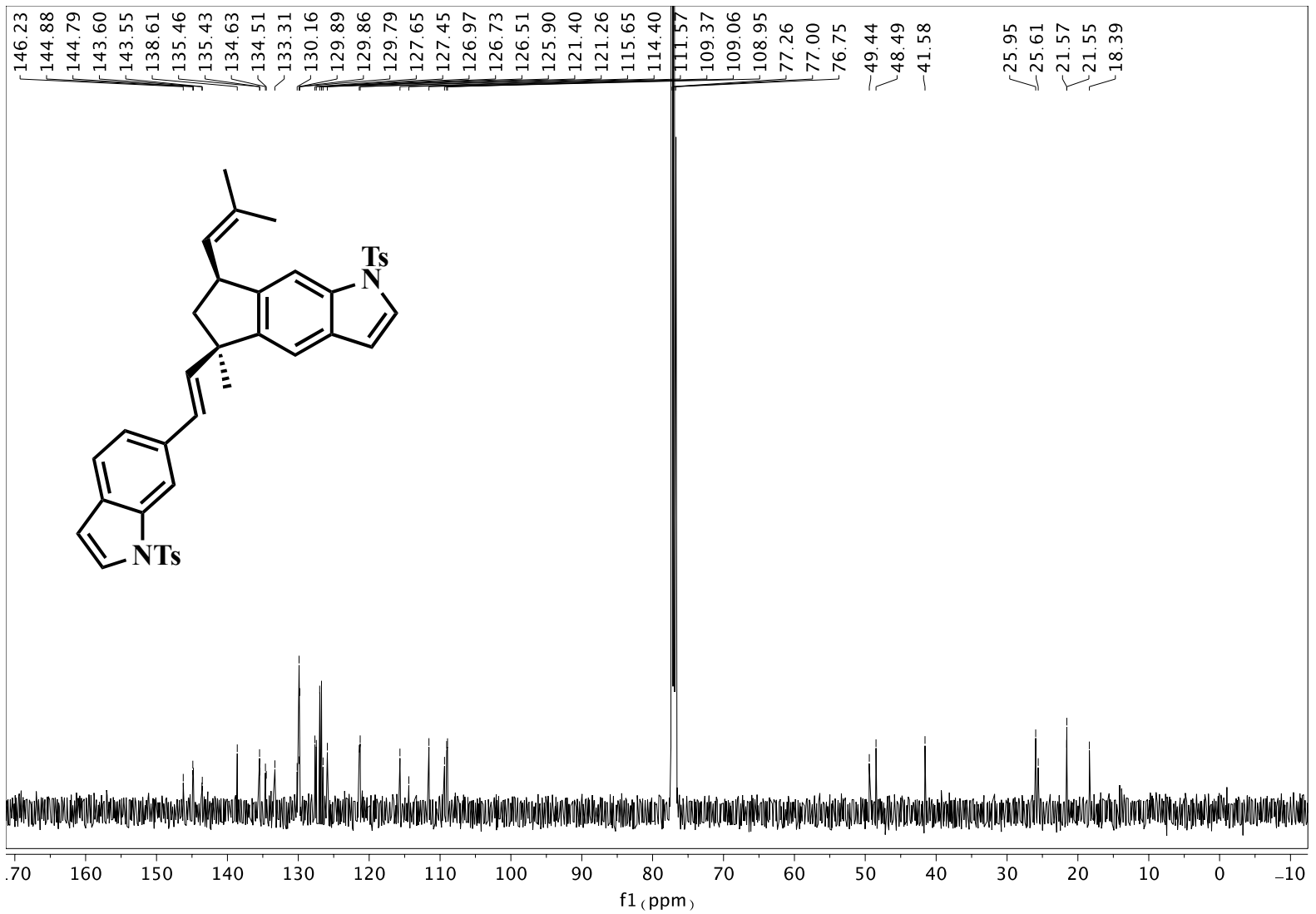

${ }^{13} \mathrm{C}$ NMR (126 MHz, $\mathrm{CDCl}_{3}$ ) Spectrum of Compound (5R,7S)-19b. 


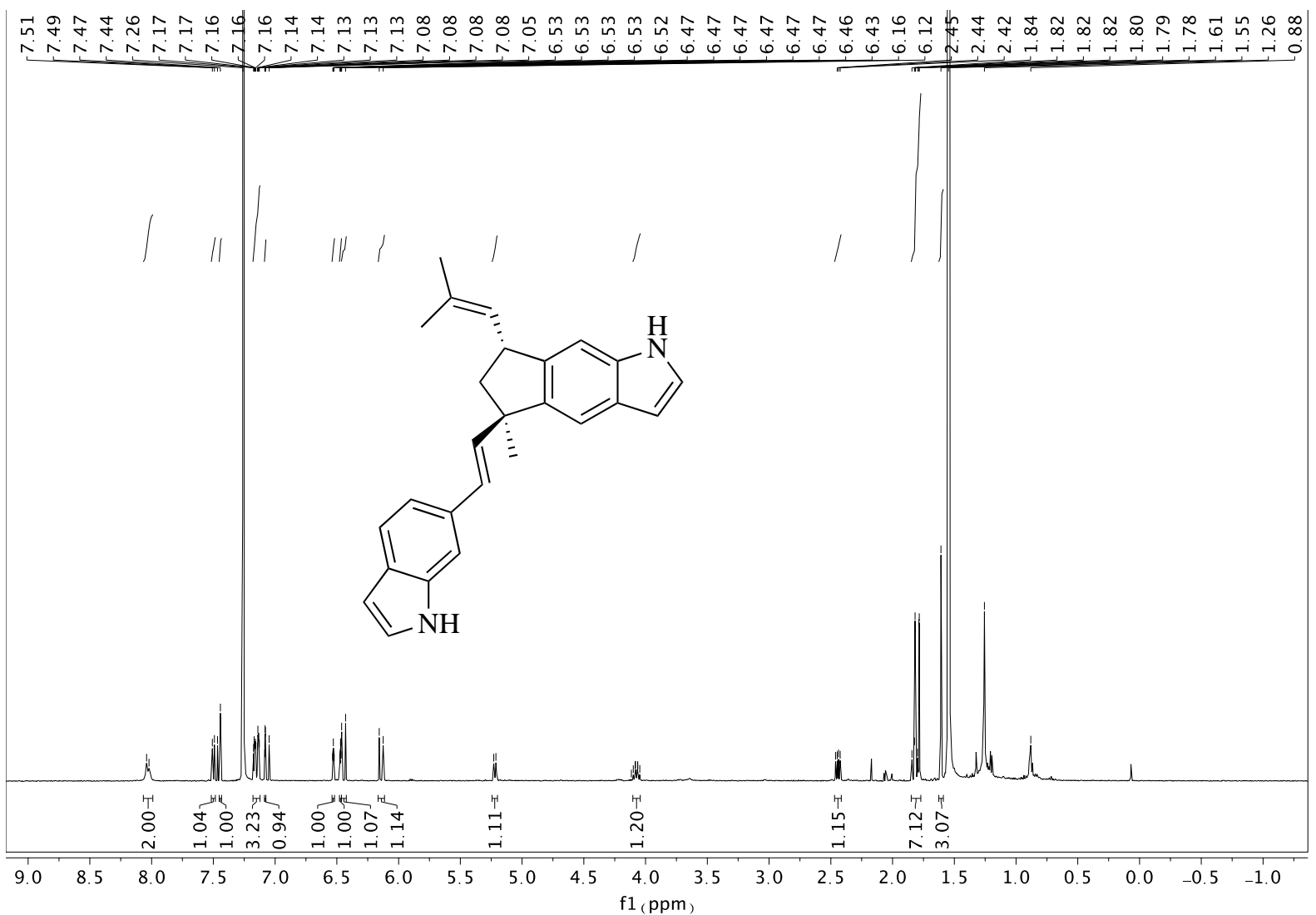

${ }^{1} \mathrm{H}$ NMR $\left(500 \mathrm{MHz}, \mathrm{CDCl}_{3}\right.$ ) Spectrum of Raputindole $\mathrm{A}(\mathbf{1})$. (residual hexanes grease: 1.26 and $0.8 \mathrm{ppm}$; residual water: $1.56 \mathrm{ppm}$ ).

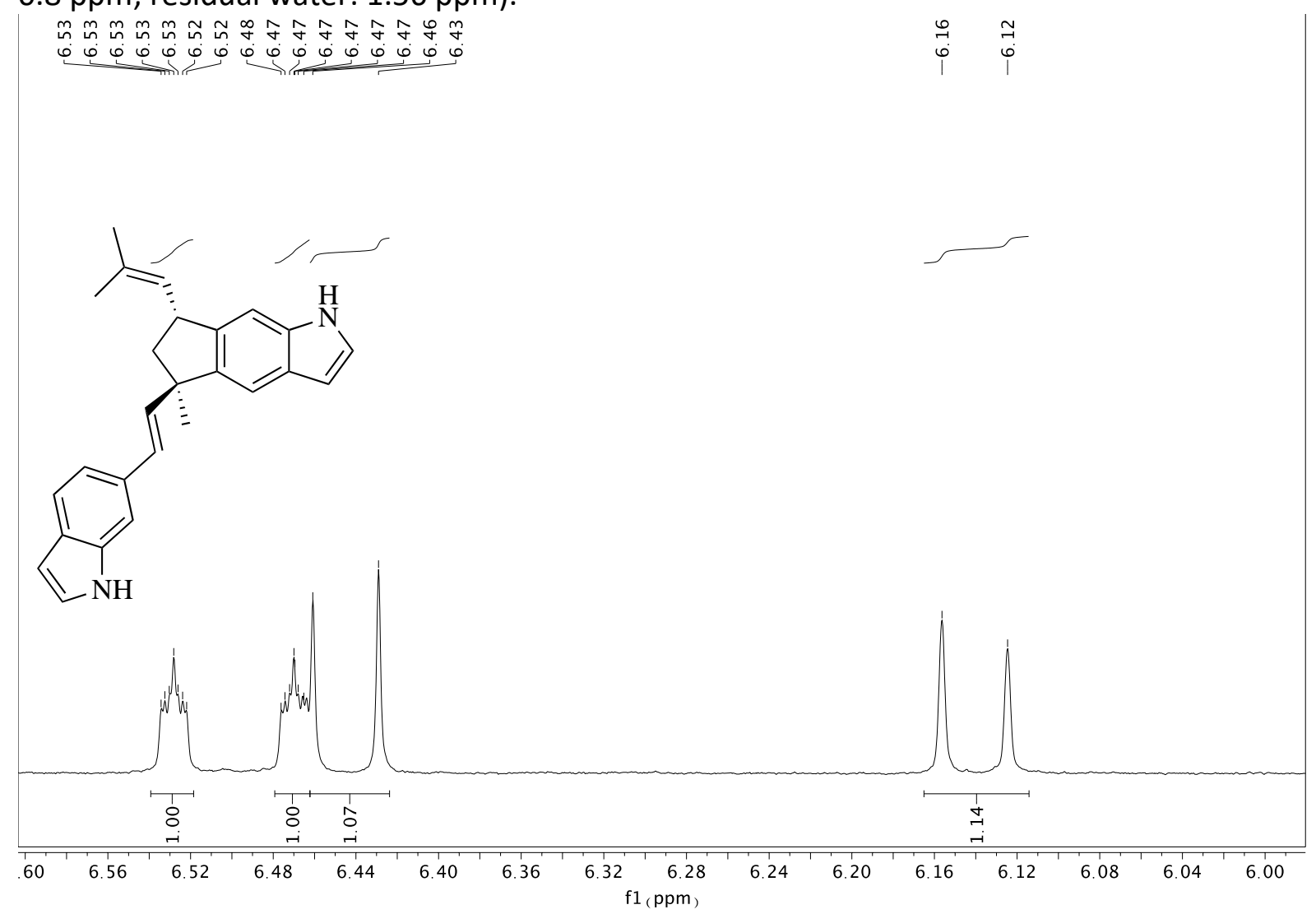

${ }^{1} \mathrm{H}$ NMR $\left(500 \mathrm{MHz}, \mathrm{CDCl}_{3}\right.$ ) Spectrum of Raputindole A (1). (residual hexanes grease: 1.26 and 0.8 ppm; residual $\left.\mathrm{H}_{2} \mathrm{O}: 1.56 \mathrm{ppm}\right)$. 


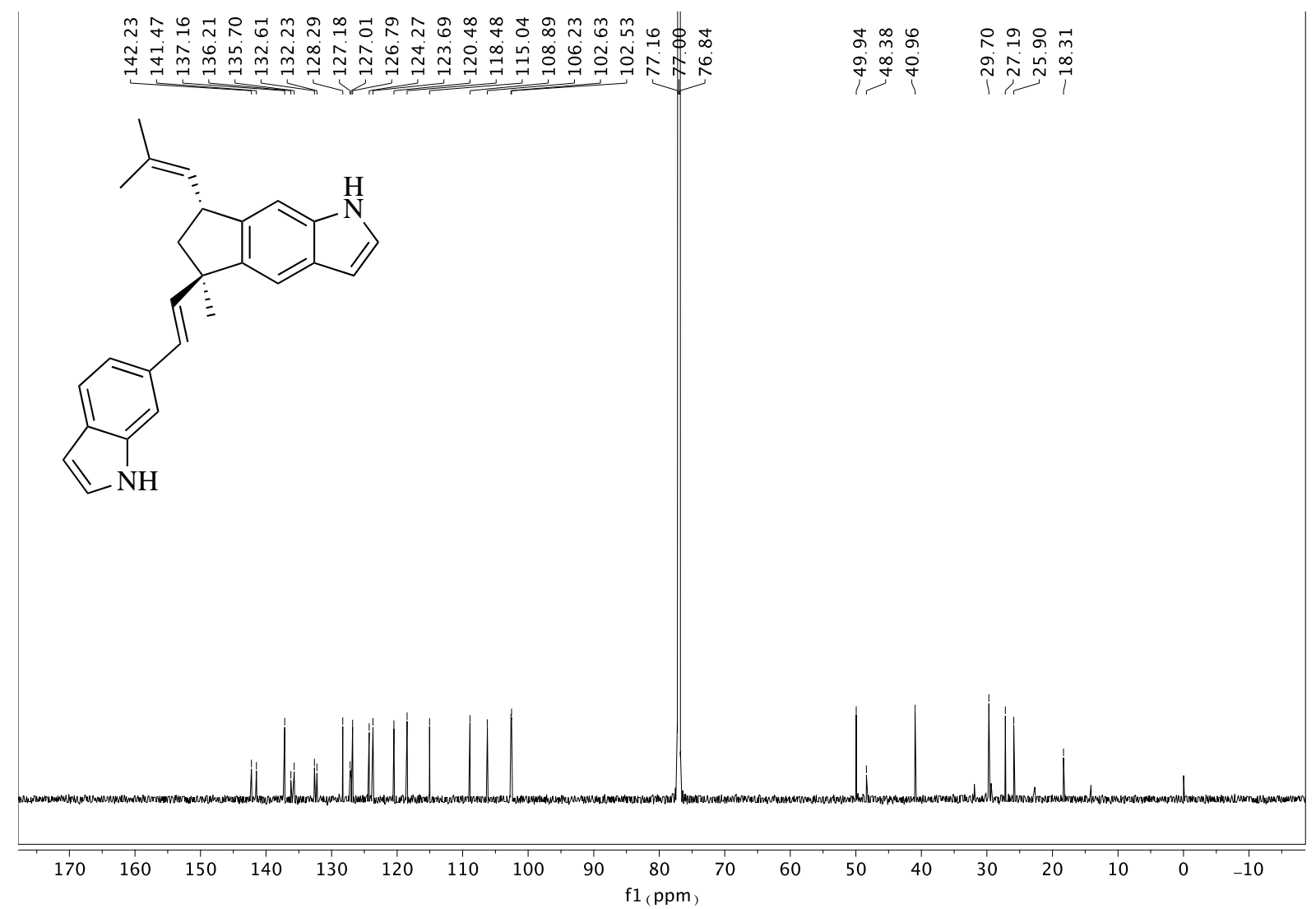

${ }^{13} \mathrm{C} \mathrm{NMR}\left(200 \mathrm{MHz}, \mathrm{CDCl}_{3}\right.$ ) Spectrum of Raputindole $\mathrm{A}$ (1). (residual hexanes grease: 29.70 ppm).

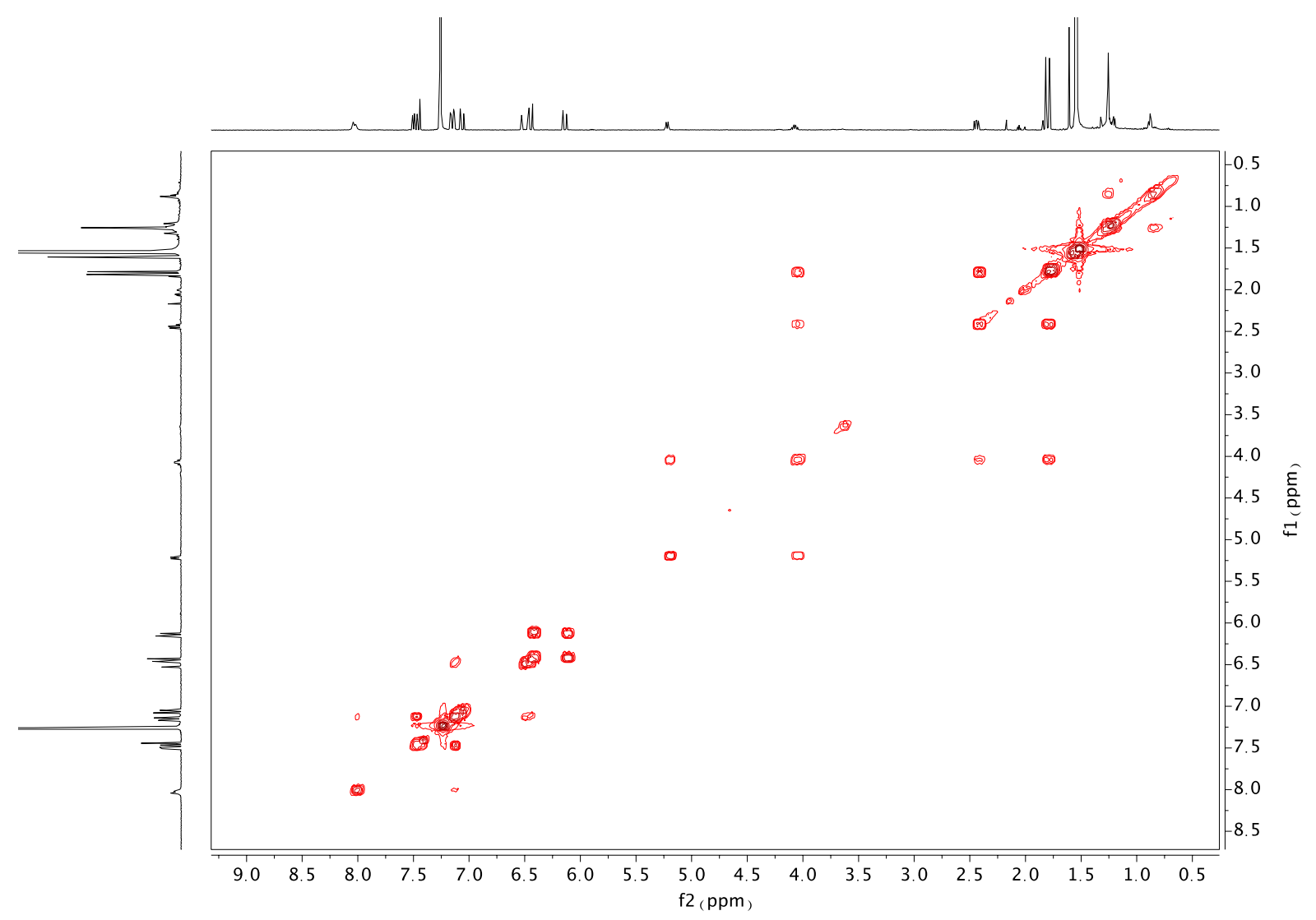

$\operatorname{COSY}(500 \mathrm{MHz})$ of Raputindole A (1). 


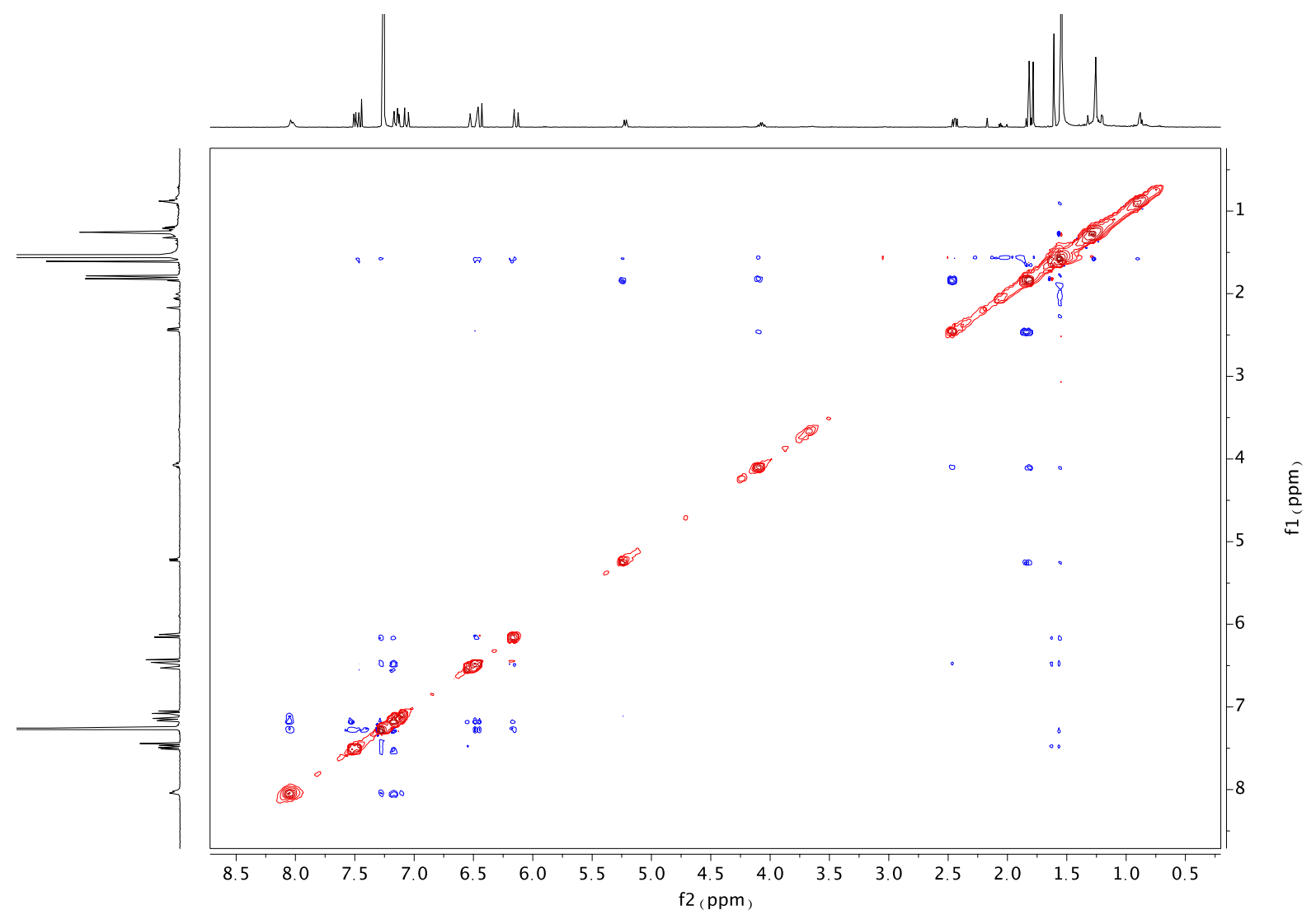

NOESY 2D (500 MHz) of Raputindole A (1).

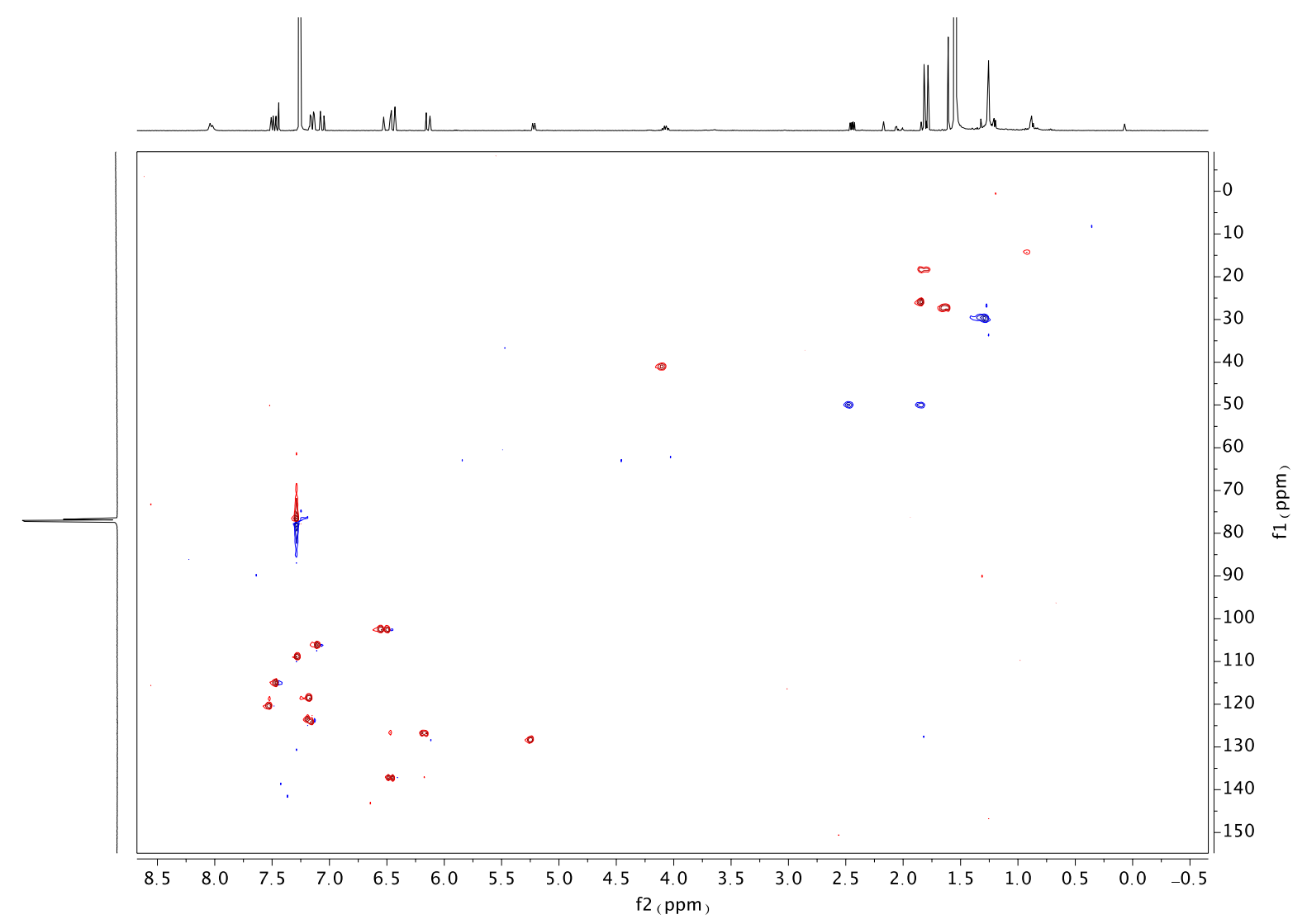

HSQC (500 MHz) of Raputindole A (1). 


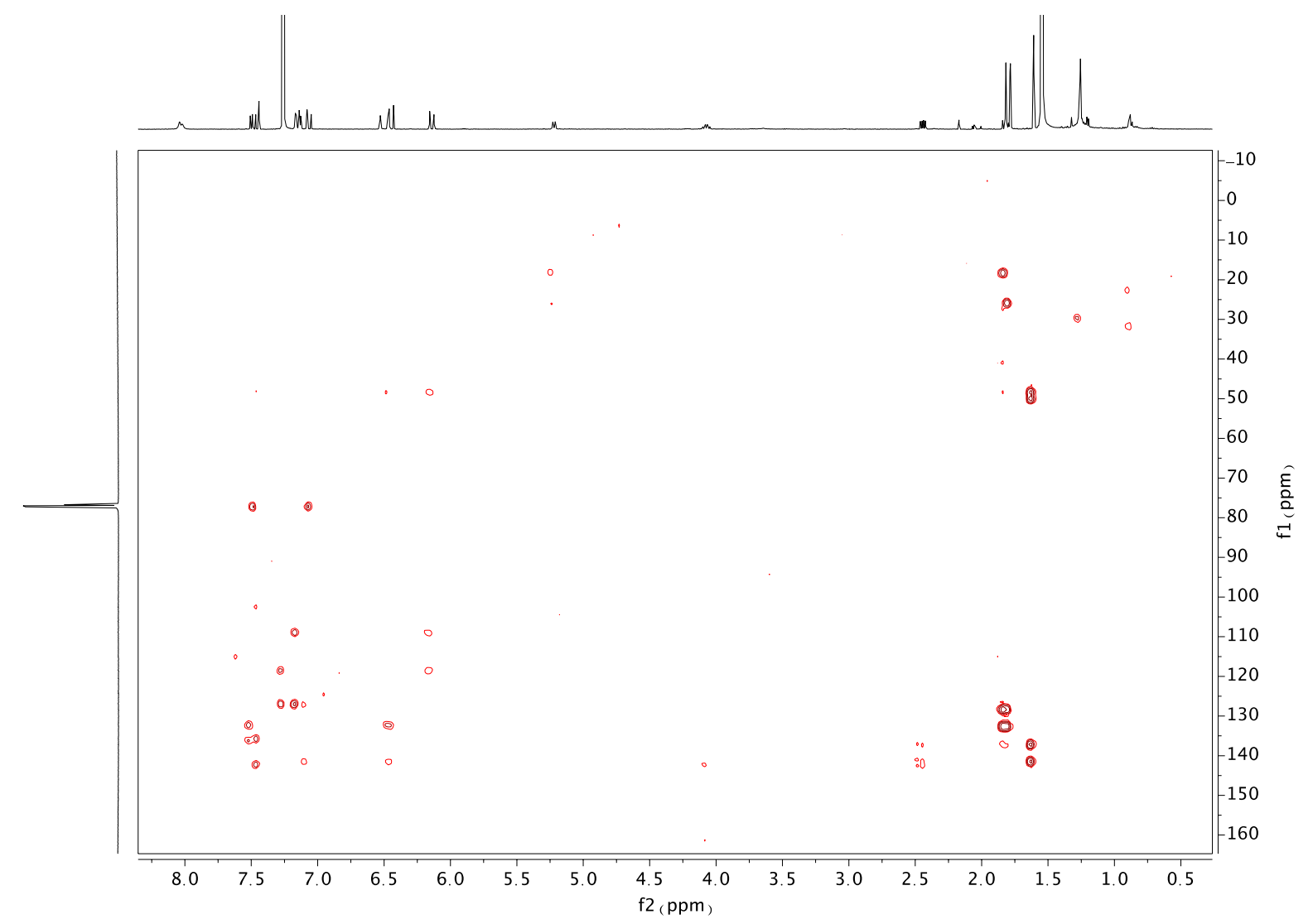

HMBC (500 MHz) of Raputindole A (1). 